\title{
The critical behavior of three-dimensional Ising spin glass models
}

\author{
Martin Hasenbusch, ${ }^{1}$ Andrea Pelissetto, ${ }^{2}$ and Ettore Vicari ${ }^{3}$ \\ 1 Institut für Theoretische Physik, Universität Leipzig, \\ Postfach 100 920, D-04009 Leipzig, Germany. \\ ${ }^{2}$ Dipartimento di Fisica dell'Università di Roma "La Sapienza" and INFN, Roma, Italy. \\ ${ }^{3}$ Dipartimento di Fisica dell'Università di Pisa and INFN, Pisa, Italy.
}

(Dated: October 24, 2018)

\begin{abstract}
We perform high-statistics Monte Carlo simulations of three-dimensional Ising spin-glass models on cubic lattices of size $L$ : the $\pm J$ (Edwards-Anderson) Ising model for two values of the disorder parameter $p, p=0.5$ and $p=0.7$ (up to $L=28$ and $L=20$, respectively), and the bond-diluted bimodal model for bond-occupation probability $p_{b}=0.45$ (up to $L=16$ ). The finite-size behavior of the quartic cumulants at the critical point allows us to check very accurately that these models belong to the same universality class. Moreover, it allows us to estimate the scaling-correction exponent $\omega$ related to the leading irrelevant operator: $\omega=1.0(1)$. Shorter Monte Carlo simulations of the bond-diluted bimodal models at $p_{b}=0.7$ and $p_{b}=0.35$ (up to $L=10$ ) and of the Ising spinglass model with Gaussian bond distribution (up to $L=8$ ) also support the existence of a unique Ising spin-glass universality class. A careful finite-size analysis of the Monte Carlo data which takes into account the analytic and the nonanalytic corrections to scaling allows us to obtain precise and reliable estimates of the critical exponents $\nu$ and $\eta$ : we obtain $\nu=2.45(15)$ and $\eta=-0.375(10)$.
\end{abstract}

PACS numbers: 75.10.Nr, 64.60.Fr, 75.40.Cx, 05.10.Ln 


\section{INTRODUCTION}

The Ising model with random ferromagnetic and antiferromagnetic couplings is a simplified model $^{1}$ for disordered uniaxial magnetic materials which show glassy behavior in some region of their phase diagram, such as $\mathrm{Fe}_{1-x} \mathrm{Mn}_{x} \mathrm{TiO}_{3}$ and $\mathrm{Eu}_{1-x} \mathrm{Ba}_{x} \mathrm{MnO}_{3}$; see, e.g., Refs. 2,3,4. The random nature of the short-ranged interactions is mimicked by nearestneighbor random bonds. This model is also interesting per se, since it provides a laboratory in which the combined effect of quenched disorder and frustration can be studied.

It is now well established that three-dimensional Ising spin-glass models present a hightemperature paramagnetic phase and, for some values of the parameters, a low-temperature glassy phase (if the frustration is small, the low-temperature phase is ferromagnetic). The two phases are separated by a continuous phase transition, which is expected to have universal features, i.e. to belong to a universality class which is independent of the details of the model and, in particular, of the disorder distribution. Several numerical works $5,6,7,8,9,10,11,12,13,14,15,16,17,18,19,20,21,22,23,24,25,26,27,28,29,30,31,32,33$ have addressed these issues, considering various Ising spin-glass models, characterized by different disorder distributions, with or without dilution. Over the years many estimates of the critical exponents have been obtained. We mention the most recent ones for the correlation-length exponent $\nu: \quad \nu=2.39(5),{ }^{30} \nu=2.72(8),{ }^{29} \nu=1.5(3),{ }^{23} \nu=1.35(10),{ }^{22} \nu=2.15(15),{ }^{21}$ $\nu=1.8(2),{ }^{20}$ obtained from simulations of the symmetric model with bimodal distribution; $\nu=2.22(15)$ for the bond-diluted symmetric bimodal model with $p_{b}=0.45 ;^{28} \nu=2.44(9)^{30}$ and $\nu=2.00(15)^{18}$ for the symmetric model with Gaussian disorder distribution; $\nu=2.4(6)$ for the random-anisotropy Heisenberg model in the limit of large anisotropy, ${ }^{27}$ which is expected to be in the same Ising spin-glass universality class. ${ }^{27,34,35}$ Moreover, the analysis of different quantities has often given different estimates of the same critical exponent, even in the same model. For instance, recent Monte Carlo (MC) studies ${ }^{29,30}$ find significant discrepancies among the estimates of the exponent $\nu$ obtained from the finite-size scaling (FSS) at $T_{c}$ of the temperature derivative of $\xi / L$, of the Binder cumulant, and of the overlap susceptibility. For the bimodal Ising model Ref. 30 quotes $\nu=2.39(5), \nu=2.79(11)$, and $\nu=1.527(8)$, from the analysis of these three quantities, respectively. A likely reason for these discrepancies is the presence of scaling corrections, which may be quite important in spin-glass systems since the absence of fast MC algorithms makes it necessary to work with systems of relatively small size.

One of the aims of the present paper is a detailed analysis of the role of scaling corrections in spin-glass systems. We present a general renormalization-group ( $R G$ ) analysis based on the Wegner expansion, ${ }^{36}$ which allows us to predict the corrections to the asymptotic critical behavior for the different quantities. In particular, we show that the analytic dependence of the relevant scaling fields on the model parameters, such as the temperature, may give rise to scaling corrections that decay as powers of $L^{-1 / \nu}$, where $L$ is the linear size of the lattice. Since $\nu \approx 2.45$ in Ising spin-glass systems, they decay quite slowly and may give rise to systematic deviations which are difficult to detect, given the small interval of values of $L$ which can be considered in MC simulations. Their presence explains some inconsistencies in the standard analyses of $\mathrm{MC}$ data reported in the literature. Thus, it is crucial to take scaling corrections into account for an accurate study of the critical behavior, for a robust check of universality among different models, and for reliable estimates of universal quantities such as the critical exponents.

In this paper we report a high-statistics MC study of different Ising spin-glass models. 
We consider the $\pm J$ Ising model for two values of the disorder parameter, the bond-diluted symmetric bimodal model with various values of the dilution, and also the model with Gaussian disorder distribution. We determine the FSS behavior of several RG invariant quantities, such as the ratio $R_{\xi} \equiv \xi / L$ ( $\xi$ is the second-moment correlation length) and the quartic cumulants defined from the overlap variables. We verify with good precision their independence on the model and disorder distribution, providing an accurate evidence of universality. Then, we obtain an estimate of the leading correction-to-scaling exponent $\omega$ : $\omega=1.0(1)$. Finally, we determine the critical exponents. We analyze the MC data at the critical point and in the high-temperature phase, taking into account the RG predictions for the scaling corrections and the precise above-reported estimate of $\omega$. We obtain

$$
\begin{aligned}
& \nu=2.45(15), \\
& \eta=-0.375(10) .
\end{aligned}
$$

Then, using scaling and hyperscaling relations we obtain

$$
\begin{aligned}
& \beta=\nu(1+\eta) / 2=0.77(5), \\
& \gamma=(2-\eta) \nu=5.8(4), \\
& \alpha=2-3 \nu=-5.4(5) .
\end{aligned}
$$

In this work we extend the results presented in Ref. 32. First, we have significantly increased the statistics of the large- $L$ data for the bimodal symmetric Ising model and we have added some data for other diluted models and for the bimodal model with Gaussian distributed couplings. Second, we present a much more detailed analysis of the critical-point data and a new analysis of the high-temperature data obtained in the parallel-tempering simulations. This allows us to check the universality of the critical behavior of the correlation length and of the susceptibility in the high-temperature phase. Finally, we discuss the extendedscaling scheme, ${ }^{29,37}$ which is inspired by the high-temperature expansion. As already noted in Ref. 29, this scheme shows an apparent improvement of the scaling behavior with respect to the naive approach in which scaling corrections are simply neglected, at least for some quantities, e.g., the overlap susceptibility. However, as we shall show, such an improvement is only marginal for the purpose of obtaining accurate results. Indeed, this requires to take into account the analytic and nonanalytic scaling corrections predicted by the RG theory.

The paper is organized as follows. In Sec. II we define the models we investigate and the quantities that are computed in the MC simulation. In Sec. III we derive the FSS predictions of the RG theory which are the basis of our FSS analyses. Some details are reported in App. A. In Sec. IV and in App. B we give some details on the MC simulations. In Sec. VA we discuss universality, verifying that the infinite-volume limit of the quartic cumulants and of $R_{\xi} \equiv \xi / L$ is independent of the model. In Sec. V B we compute the leading correction-to-scaling exponent $\omega$. In Sec. VI we compute the critical exponents $\nu$ and $\eta$ and the critical temperature for the different models by using the data close to the critical point. In Sec. VII we present a global analysis of all available high-temperature data obtained in our parallel-tempering MC simulations. We again determine the critical exponents and show that the FSS behavior of several quantities is universal. Moreover, we discuss the extendedscaling scheme of Ref. 29. In Sec. VIII we compute the high-temperature zero-momentum quartic couplings. Finally, in Sec. IX we present our conclusions. 


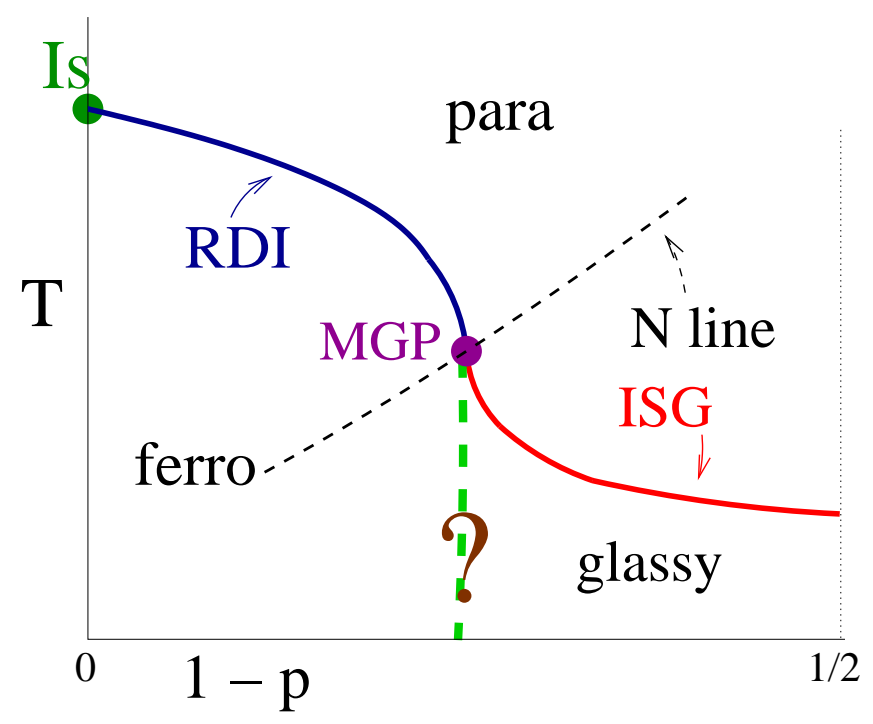

FIG. 1: Phase diagram of the three-dimensional $\pm J$ (Edwards-Anderson) Ising model in the $T-p$ plane for $p \geq 1 / 2$, i.e., $1-p \leq 1 / 2$. The phase diagram is symmetric under $p \rightarrow 1-p$.

\section{ISING SPIN GLASS SYSTEMS}

\section{A. The $\pm J$ Edwards-Anderson Ising model and its phase diagram}

We consider the $\pm J$ Edwards-Anderson Ising model on a simple cubic lattice of linear size $L$ with periodic boundary conditions. The corresponding Hamiltonian is ${ }^{1}$

$$
H=-\sum_{\langle x y\rangle} J_{x y} \sigma_{x} \sigma_{y},
$$

where $\sigma_{x}= \pm 1$, the sum is over the nearest-neighbor lattice sites, and the exchange interactions $J_{x y}$ are uncorrelated quenched random variables with probability distribution

$$
P\left(J_{x y}\right)=p \delta\left(J_{x y}-1\right)+(1-p) \delta\left(J_{x y}+1\right) .
$$

The usual bimodal Ising spin glass model, for which $\left[J_{x y}\right]=0$ (brackets indicate the average over the disorder distribution), corresponds to $p=1 / 2$. For $p \neq 1 / 2$ we have

$$
\left[J_{x y}\right]=2 p-1 \neq 0
$$

and ferromagnetic (or antiferromagnetic) configurations are energetically favored. Note that the free energy and also the correlations of the overlap variables that we shall define below are invariant under $p \rightarrow 1-p$ and thus we can always assume $p \geq 1 / 2$.

The $T$ - $p$ phase diagram of the three-dimensional $\pm J$ Ising model is sketched in Fig. 1. The high-temperature phase is paramagnetic for any $p$. The nature of the low-temperature phase depends on the value of $p$ : it is ferromagnetic for small values of $1-p$, while it is glassy with vanishing magnetization for sufficiently large values of $1-p$. The three phases are separated by transition lines, which meet at a magnetic-glassy multicritical point (MGP), 
usually called Nishimori point, which is located along the so-called Nishimori line $\mathrm{l}^{38,39,40,41,42}$ defined by the relation $(p \geq 1 / 2)$

$$
T=T_{N}(p), \quad T_{N}(p)=\frac{2}{\ln \frac{p}{1-p}} .
$$

On the Nishimori line the magnetic and the overlap two-point correlation functions are equal.

The paramagnetic-ferromagnetic $(\mathrm{PF})$ transition line starts at the Ising transition at $p=1$ and extends up to the MGP at $p=p^{*}$. For $p=1$ there is the standard Ising transition at $^{43} T_{\mathrm{Is}}=4.5115248(14)$. Disorder is a relevant perturbation of the pure three-dimensional Ising fixed point. As a consequence, the Ising point $p=1$ is a multicritical point ${ }^{44}$ with crossover exponent $\phi=\alpha_{\text {Is }}$, where ${ }^{45} \alpha_{\text {Is }}=0.1096(5)$ is the Ising specific-heat exponent. The critical behavior for any $1>p>p^{*}$ belongs to the randomly-dilute Ising (RDI) universality class, ${ }^{44}$ characterized by the correlation-length critical exponent ${ }^{46,47} \nu=0.683(2)$ and by the magnetic exponent $\eta=0.036(1)$. The coordinates of the MGP in the $T-p$ plane are ${ }^{42}$ $T^{*}=1.6692(3)$ and $p^{*}=0.76820(4)$. The multicritical behavior is characterized by ${ }^{42}$ the thermal exponent $\nu=1.64(5)$, the crossover exponent $\phi=1.67(10)$, and the magnetic (and also overlap) exponent $\eta=-0.114(3)$.

The paramagnetic-glassy $(\mathrm{PG})$ transition line starts at the MGP and extends up to $p=$ $1 / 2$ (actually up to $p=1-p^{*}=0.23180(4)$ due to the symmetry $p \rightarrow 1-p$ of the phase diagram). A reasonable hypothesis is that the $\mathrm{PG}$ critical behavior is independent of $p$, as found in mean-field models. ${ }^{48}$ Hence, for any $p^{*}>p>1-p^{*}$ the $\mathrm{PG}$ transition is expected to belong to the same universality class (named ISG in Fig. 1) as that of the bimodal Ising spin-glass model at $p=1 / 2$. The critical behavior along this transition line is the main subject of this paper. As we shall see, the universality hypothesis is fully confirmed by our FSS analyses at $p=0.5$ and $p=0.7$.

The nature of the ferromagnetic-glassy (FG) transition line is not clear yet. At fixed $p$ the following inequality holds: ${ }^{38,49}$

$$
\left|\left[\left\langle\sigma_{x} \sigma_{y}\right\rangle_{T}\right]\right| \leq\left[\left|\left\langle\sigma_{x} \sigma_{y}\right\rangle_{T_{N}(p)}\right|\right]
$$

where the subscripts indicate the temperature of the thermal average, and $T_{N}(p)$ is the temperature along the Nishimori line, defined in Eq. (6). This relation shows that ferromagnetism can only exist in the region $p>p^{*}$ and that the system is maximally magnetized along the Nishimori line. Ref. 50 (see also Refs. 41,51) also argues that the FG transition line coincides with the line $p=p^{*}$, from $T=T^{*}$ to $T=0$. Recent numerical investigations $^{52,53,54}$ of the two-dimensional $\pm J$ model have shown that this conjecture is not exact, though quantitative deviations are small: at $T=0$ the critical value $p_{c}$ where ferromagnetism disappears is definitely larger than $p^{*}$, indicating a reentrant transition line. In three dimensions Ref. 55 quotes $p_{c}=0.778(5)$, which is slightly larger than $p^{*}=0.76820(4)$, with an associated critical exponent $\nu=1.1(3)$ : it is therefore likely that the conjecture does not hold in three dimensions as well. We also mention that a mixed low-temperature phase, ${ }^{56}$ in which ferromagnetism and glassy order coexist, is found in mean-field models ${ }^{48}$ such as the infinite-range Sherrington-Kirkpatrick model. ${ }^{57}$ Its presence has been confirmed in the $\pm J$ Ising model on a Bethe lattice. ${ }^{58}$ However, there is no evidence of a mixed phase in the $\pm J$ Ising model on a cubic lattice ${ }^{55}$ and in related models. ${ }^{59}$ In particular, the numerical results of Ref. 55 show that the onset of the glassy behavior at $T=0$ occurs close to the point where the ferromagnetic phase disappears, and are consistent with a single transition 
within numerical precision. Nevertheless, the existence of such a mixed phase is still an open problem, as discussed in Ref. 58.

\section{B. Other Ising spin-glass models}

We also consider the bond-diluted bimodal Ising model (BDBIM) defined by Hamiltonian (3) with bond probability distribution

$$
P\left(J_{x y}\right)=p_{b}\left[\frac{1}{2} \delta\left(J_{x y}-J\right)+\frac{1}{2} \delta\left(J_{x y}+J\right)\right]+\left(1-p_{b}\right) \delta\left(J_{x y}\right) .
$$

A PG transition occurs for sufficiently small values of $1-p_{b}$, i.e. for $p_{b}>p_{S G}$. While investigations at $T=0$ indicate that $p_{S G}$ should be identified with the bond-percolation point $^{60,61}\left(p_{\text {perc }}=0.2488126(5)\right.$ on a simple cubic lattice $\left.{ }^{62}\right)$, recent investigations of the critical behavior close to the percolation point suggest that $p_{S G}$ is larger than $p_{\text {perc }}{ }^{63}$

The model can be extended by considering the distribution

$$
P\left(J_{x y}\right)=p_{b}\left[p \delta\left(J_{x y}-J\right)+(1-p) \delta\left(J_{x y}+J\right)\right]+\left(1-p_{b}\right) \delta\left(J_{x y}\right) .
$$

In this case, for $p_{b}>p_{S G}\left(p_{S G}\right.$ may depend on $\left.p\right)$ we expect a $T$ - $p$ phase diagram similar to the one sketched in Fig. 1 for the $\pm J$ Ising model without dilution, with a PF and a PG transition line meeting at a multicritical point.

A PG transition is also observed in the random-bond Ising spin-glass model with Gaussian bond distribution:

$$
P\left(J_{x y}\right)=\frac{1}{\sqrt{2 \pi}} e^{-J_{x y}^{2} / 2} .
$$

This transition is expected to be in the same universality class as that of the bimodal Ising model. ${ }^{30}$

\section{Overlap thermodynamic quantities}

In this work we focus on the critical behavior of the overlap parameter

$$
q_{x} \equiv \sigma_{x}^{(1)} \sigma_{x}^{(2)}
$$

where the spins $\sigma_{x}^{(i)}$ belong to two independent replicas with the same disorder realization $\left\{J_{x y}\right\}$. The corresponding correlation function is

$$
G(x) \equiv\left[\left\langle q_{0} q_{x}\right\rangle\right]=\left[\left\langle\sigma_{0} \sigma_{x}\right\rangle^{2}\right]
$$

where the angular and square brackets indicate the thermal average and the quenched average over $\left\{J_{x y}\right\}$, respectively. We define the susceptibility $\chi$ and the second-moment correlation length $\xi$ as

$$
\begin{aligned}
\chi & \equiv \sum_{x} G(x), \\
\xi^{2} & \equiv \frac{1}{4 \sin ^{2}\left(p_{\min } / 2\right)} \frac{\widetilde{G}(0)-\widetilde{G}(p)}{\widetilde{G}(p)},
\end{aligned}
$$


where $p=\left(p_{\min }, 0,0\right), p_{\min } \equiv 2 \pi / L$, and $\widetilde{G}(q)$ is the Fourier transform of $G(x)$.

We also define the $\mathrm{RG}$ invariant quantities

$$
\begin{aligned}
R_{\xi} & \equiv \xi / L, \\
U_{4} & \equiv \frac{\left[\mu_{4}\right]}{\left[\mu_{2}\right]^{2}}, \\
U_{22} & \equiv \frac{\left[\mu_{2}^{2}\right]-\left[\mu_{2}\right]^{2}}{\left[\mu_{2}\right]^{2}},
\end{aligned}
$$

where

$$
\mu_{k} \equiv\left\langle\left(\sum_{x} q_{x}\right)^{k}\right\rangle
$$

We call them phenomenological couplings and denote them by $R$ in the following.

In the high-temperature paramagnetic phase, we also consider the zero-momentum quartic couplings

$$
\begin{aligned}
& G_{4} \equiv-\frac{\chi_{4}}{\xi^{3} \chi^{2}}, \\
& G_{22} \equiv-\frac{\chi_{22}}{\xi^{3} \chi^{2}}
\end{aligned}
$$

where

$$
\begin{aligned}
& \chi_{4} \equiv \frac{1}{L^{3}}\left(\left[\mu_{4}\right]-3\left[\mu_{2}^{2}\right]\right), \\
& \chi_{22} \equiv \frac{1}{L^{3}}\left(\left[\mu_{2}^{2}\right]-\left[\mu_{2}\right]^{2}\right) .
\end{aligned}
$$

The critical limit $T \rightarrow T_{c}^{+}$of the zero-momentum quartic couplings $G_{4}$ and $G_{22}$ is universal.

\section{FINITE-SIZE SCALING}

In this section we summarize some basic results concerning FSS, which allow us to understand the role of the analytic and nonanalytic scaling corrections. We consider two Ising spin-glass systems coupled by an interaction

$$
h \sum_{x} q_{x}=h \sum_{x} \sigma_{x}^{(1)} \sigma_{x}^{(2)}
$$

where $h$ is a constant external field. The model is defined on a cubic lattice of linear size $L$ with periodic boundary conditions.

By applying standard RG arguments we expect the disorder-averaged free-energy density to be the sum of a regular part and a singular part:

$$
\mathcal{F}(\beta, h, L)=\mathcal{F}_{\text {reg }}(\beta, h, L)+\mathcal{F}_{\text {sing }}(\beta, h, L),
$$

where $\beta \equiv 1 / T$. The regular part is expected to depend on $L$ only through exponentially small terms, while the singular part encodes the critical behavior. The starting point of FSS 
is the scaling behavior of the singular part of the free-energy density (see, e.g., Refs. 36,47, $64,65)$ :

$$
\mathcal{F}_{\text {sing }}(\beta, h, L)=L^{-d} F\left(u_{h} L^{y_{h}}, u_{t} L^{y_{t}},\left\{v_{i} L^{y_{i}}\right\}\right),
$$

where $d$ is the space dimension, $u_{h}$ and $u_{t}$ are the scaling fields associated with $h$ and the reduced temperature $t \sim 1-\beta / \beta_{c}$ (their RG dimensions are $y_{h}=(d+2-\eta) / 2$ and $y_{t}=1 / \nu$, respectively), and $v_{i}$ are irrelevant scaling fields with $y_{i}<0$. At the critical point we have $u_{t}(t=0, h=0)=0$ and $u_{h}(t=0, h=0)=0$, while, generically, we expect $v_{i}(t=0, h=0) \neq 0$. Since $y_{i}<0$, for large $L$ the free energy can be expanded in powers of $\left\{v_{i} L^{y_{i}}\right\}$. Therefore, we can write

$$
\mathcal{F}_{\text {sing }}(\beta, h, L)=L^{-d} f\left(u_{h} L^{y_{h}}, u_{t} L^{y_{t}}\right)+v_{\omega} L^{-d-\omega} f_{\omega}\left(u_{h} L^{y_{h}}, u_{t} L^{y_{t}}\right)+\ldots
$$

where the leading nonanalytic correction-to-scaling exponent $\omega$ is related to the RG dimension $y_{\omega}$ of the leading irrelevant scaling field $v_{\omega} \equiv v_{1}, \omega=-y_{\omega}$. The scaling fields are analytic functions of the system parameters - in particular, of $h$ and $t$-and are expected not to depend on $L$. Note also that the size $L$ is expected to be an exact scaling field for periodic boundary conditions. For a general discussion of these issues, see Ref. 65, Sec. III of Ref. 66, and references therein. In general, $u_{t}$ and $u_{h}$ can be expanded as

$$
\begin{aligned}
& u_{h}=h \bar{u}_{h}(t)+O\left(h^{3}\right), \quad \bar{u}_{h}(t)=a_{h}+a_{1} t+O\left(t^{2}\right), \\
& u_{t}=c_{t} t+c_{02} t^{2}+c_{20} h^{2}+c_{21} h^{2} t+O\left(t^{3}, h^{4}, h^{4} t\right),
\end{aligned}
$$

where we used the fact that the free energy is symmetric under $h \rightarrow-h$. In the expansion of $u_{h, t}$ around the critical point $h, t=0$, the terms beyond the leading ones give rise to analytic scaling corrections.

The scaling behavior of zero-momentum thermodynamic quantities can be obtained by performing appropriate derivatives of $\mathcal{F}$ with respect to $h$. For instance, for the overlap susceptibility at $h=0$ we obtain

$$
\chi(\beta, L)=\left.\frac{\partial^{2} \mathcal{F}}{\partial h^{2}}\right|_{h=0}=L^{2-\eta} \bar{u}_{h}(t)^{2} g\left(u_{t} L^{y t}\right)\left[1+v_{\omega} L^{-\omega} g_{\omega}\left(u_{t} L^{y_{t}}\right)+\ldots\right]+g_{\mathrm{reg}}(\beta) .
$$

The function $g_{\text {reg }}(\beta)$ represents the contribution of the regular part $\mathcal{F}_{\text {reg }}(\beta, h, L)$ of the freeenergy density and is $L$ independent (apart from exponentially small terms). It gives rise to a correction proportional to $L^{\eta-2}$. Analogous formulae hold for the $2 n$-point susceptibilities.

The FSS of the phenomenological couplings is given by

$$
\begin{aligned}
R(\beta, L) & =r\left(u_{t} L^{y_{t}}\right)+v_{\omega} r_{\omega}\left(u_{t} L^{y_{t}}\right) L^{-\omega}+\ldots \\
& =R^{*}+r^{\prime}(0) c_{t} t L^{y_{t}}+\ldots+c_{\omega} L^{-\omega}+\ldots
\end{aligned}
$$

where $R^{*} \equiv r(0), c_{\omega}=v_{\omega} r_{\omega}(0)$, and the second line holds only very close to the critical point, for $t L^{y_{t}} \ll 1$. A proof of Eq. (30) for the phenomenological couplings $U_{4}, U_{22}$, and $R_{\xi}$ is presented in App. A.

The thermal RG exponent $y_{t}=1 / \nu$ is usually computed from the FSS of the derivative $R^{\prime}$ of a phenomenological coupling $R$ with respect to $\beta$ at $\beta_{c}$. Using Eq. (30) one obtains

$$
R^{\prime} \equiv \frac{\partial R}{\partial \beta}=L^{y_{t}}\left(\partial_{\beta} u_{t}\right)\left[r^{\prime}\left(u_{t} L^{y_{t}}\right)+v_{\omega} L^{-\omega} r_{\omega}^{\prime}\left(u_{t} L^{y_{t}}\right)+\cdots\right]
$$


One may also consider the derivative $\chi^{\prime} \equiv d \chi / d \beta$ of the susceptibility $\chi$. From Eq. (29) we obtain

$$
\begin{aligned}
\chi^{\prime}= & L^{2-\eta+y_{t}} \bar{u}_{h}^{2} \partial_{\beta} u_{t}\left\{g^{\prime}\left(u_{t} L^{y_{t}}\right)+v_{\omega} L^{-\omega}\left[g^{\prime}\left(u_{t} L^{y_{t}}\right) g_{\omega}\left(u_{t} L^{y_{t}}\right)+g\left(u_{t} L^{y_{t}}\right) g_{\omega}^{\prime}\left(u_{t} L^{y_{t}}\right)\right]\right\} \\
& +2 L^{2-\eta} \bar{u}_{h} \partial_{\beta} \bar{u}_{h} g\left(u_{t} L^{y_{t}}\right)+\cdots+g_{\mathrm{reg}}^{\prime}(\beta) .
\end{aligned}
$$

Note that the second term in the right hand side gives rise to scaling corrections proportional to $L^{-y_{t}}=L^{-1 / \nu}$, while the background term $g_{\text {reg }}^{\prime}(\beta)$ leads to corrections proportional to $L^{-y_{t}-2+\eta}$.

At $T=T_{c}$, setting $t=0$ in the above-reported equations, we obtain:

$$
\begin{aligned}
& R=R^{*}+c_{\omega} L^{-\omega}+\ldots, \\
& \chi=c L^{2-\eta}\left(1+c_{\omega} L^{-\omega}+\ldots\right), \\
& R^{\prime}=c L^{1 / \nu}\left(1+c_{\omega} L^{-\omega}+\ldots\right), \\
& \chi^{\prime}=c L^{2-\eta+1 / \nu}\left(1+c_{\omega} L^{-\omega}+\ldots+c_{a} L^{-1 / \nu}+\ldots\right) .
\end{aligned}
$$

Note that, unlike the temperature derivative $R^{\prime}$ of a RG-invariant quantity, $\chi^{\prime}$ also presents an $L^{-1 / \nu}$ scaling correction, due to the analytic dependence on $t$ of the scaling field $u_{h}$ (for this reason we call it analytic correction). Since, as we shall see, in the Ising spin-glass case $1 / \nu \approx 0.4$ and $\omega \approx 1.0$, the scaling corrections in $\chi^{\prime}$ decay significantly more slowly than those occurring in $R^{\prime}$. This makes the ratio

$$
\frac{\chi^{\prime}}{\chi} \sim L^{1 / \nu}
$$

unsuitable for a precise determination of $\nu$ and explains the significant discrepancies observed in Ref. 30.

Instead of computing the various quantities at fixed Hamiltonian parameters, one can also consider FSS keeping a phenomenological coupling $R$ fixed at a given value $R_{f} \cdot{ }^{46,67}$ This means that, for each $L$, one determines $\beta_{f}\left(L, R_{f}\right)$, such that $R\left(\beta=\beta_{f}\left(L, R_{f}\right), L\right)=R_{f}$, and then considers any quantity at $\beta=\beta_{f}\left(L, R_{f}\right)$. The value $R_{f}$ can be specified at will, as long as $R_{f}$ is taken between the high- and low-temperature fixed-point values of $R$. For $R_{f} \neq R^{*}$, where $R^{*}$ is defined in Eq. (33), $\beta_{f}$ converges to $\beta_{c}$ as

$$
\beta_{f}-\beta_{c} \sim L^{-1 / \nu}
$$

while for $R_{f}=R^{*}$ we have

$$
\beta_{f}-\beta_{c} \sim L^{-1 / \nu-\omega}
$$

Indeed, if $u_{t, f}\left(L, R_{f}\right)$ is the value of $u_{t}$ for $\beta=\beta_{f}\left(L, R_{f}\right)$, we obtain from Eq. (30)

$$
u_{t, f} L^{y_{t}}=B\left(R_{f}\right)+v_{\omega} B_{\omega}\left(R_{f}\right) L^{-\omega}+\cdots
$$

where, using Eq. (30),

$$
\begin{aligned}
r(B(x)) & =x, \\
B_{\omega}(x) & =-\frac{r_{\omega}(B(x))}{r^{\prime}(B(x))} .
\end{aligned}
$$


Now, if $R_{f}=R^{*}$, we have $B\left(R_{f}\right)=0$, which implies $u_{t, f} \sim L^{-y_{t}-\omega}$, hence Eq. (39). Otherwise, $B\left(R_{f}\right)$ is different from zero and we obtain the behavior (38).

If we now substitute relation (40) into Eqs. (29), (30), (31), and (32), we obtain the expansion of the different quantities at fixed $R_{f}$, which we denote by adding a bar: given $\mathcal{O}(\beta, L)$, we define $\overline{\mathcal{O}}\left(L, R_{f}\right) \equiv \mathcal{O}\left[\beta_{f}\left(L, R_{f}\right), L\right]$. For $R_{f}=R^{*}$, since $u_{t, f} \sim L^{-y_{t}-\omega}$ we reobtain Eqs. (33), (34), (35), and (36), with different coefficients, of course. If $R_{f} \neq R^{*}$, we must be more careful. The behavior of another phenomenological coupling $R_{\alpha}$ does not change qualitatively and we still have

$$
\bar{R}_{\alpha}\left(L, R_{f}\right) \approx \bar{R}_{\alpha}^{*}+\bar{c}_{\alpha} L^{-\omega}+\ldots,
$$

where $\bar{R}_{\alpha}^{*}=r_{\alpha}\left[B\left(R_{f}\right)\right]$ is universal. It depends on $R_{f}$ and satisfies $\bar{R}_{\alpha}^{*}=R_{\alpha}^{*}$ for $R_{f}=R^{*}$. Also $\bar{\chi}^{\prime}$ behaves as it does at fixed $T=T_{c}$, i.e. it follows Eq. (36). On the other hand, $\bar{\chi}$ and $\bar{R}^{\prime}$ present additional analytic corrections. Indeed, since [see Eq. (27)]

$$
\bar{u}_{h, f}=a_{h}+\frac{a_{1}}{c_{t}} B\left(R_{f}\right) L^{-y_{t}}+\cdots
$$

(nonanalytic $O\left(L^{-\omega}\right)$ corrections have been neglected), Eq. (29) gives

$$
\bar{\chi}_{\alpha}\left(L, R_{f}\right)=L^{2-\eta}\left[a_{h}^{2}+2 \frac{a_{1} a_{h}}{c_{t}} B\left(R_{f}\right) L^{-y_{t}}+O\left(L^{-2 y_{t}}\right)\right] g\left(B\left(R_{f}\right)\right)\left[1+O\left(L^{-\omega}\right)\right] .
$$

If $R_{f} \neq R^{*}, B\left(R_{f}\right) \neq 0$, and thus analytic corrections occur. Note that, if $R_{f}$ is close to $R^{*}$, since $B\left(R^{*}\right)=0$, we have

$$
B\left(R_{f}\right) \sim R_{f}-R^{*}
$$

Hence, in this case the analytic corrections are small, of order $\left(R_{f}-R^{*}\right) L^{-1 / \nu}$. In general, corrections of order $L^{-k / \nu}$ have amplitudes proportional to $\left(R_{f}-R^{*}\right)^{k}$.

\section{MONTE CARLO SIMULATIONS}

In the MC simulations we employed the Metropolis algorithm, the random-exchange method (often called parallel-tempering or multiple Markov-chain method), ${ }^{68}$ and multispin coding. See App. B for details on their implementation.

We simulated the $\pm J$ Ising model at $p=0.5$ for $L=3-14,16,20,24,28$, at $p=0.7$ for $L=3-12,14,16,20$, and the BDBIM at $p_{b}=0.45$ for $L=4-12,14,16$. We averaged over a large number $N_{s}$ of disorder samples: $N_{s} \approx 6.4 \cdot 10^{6}$ up to $L=12, N_{s} / 10^{3} \approx$ $2400,2800,1500,245,150,18$, respectively for $L=13,14,16,20,24,28$ in the case of the $\pm J$ Ising model at $p=0.5$. Similar statistics were collected at $p=0.7$ (except for $L=20$ where the statistics were approximately $1 / 3$ of those for $p=0.5$ ), while for the BDBIM statistics were smaller (typically, by a factor of two for the small lattices and by a factor of 6 for the largest ones). We also considered the BDBIM at $p_{b}=0.7$ and $p_{b}=0.35$ and the Ising model with Gaussian distributed couplings, but only performed simulations for small values of $L$ : $L=4,6,8,10$ for the BDBIM and $L=4,5,6,8$ for the Gaussian model.

For each $L$ and model we performed parallel-tempering runs. This allowed us to estimate the different quantities in a large interval $\left[\beta_{\min }, \beta_{\max }\right]$. To fix $\beta_{\max }$ we used the results of Ref. 30, which provided the best estimates of $R_{\xi}^{*}$ at the time we started our simulations: 
TABLE I: Quartic cumulant $\bar{U}_{4}$ at fixed $R_{\xi}=0.63$ for the $\pm J$ Ising model at $p=0.5$ and $p=0.7$, for the BDBIM at $p_{b}=0.45,0.7,0.35$, and for the Ising spin-glass model with Gaussian bond distribution.

\begin{tabular}{rlllllc}
\hline \hline$L$ & \multicolumn{1}{c}{ $\pm J_{p=0.5}$} & $\pm J_{p=0.7}$ & $\mathrm{BDBIM}_{p_{b}=0.45}$ & $\mathrm{BDBIM}_{p_{b}=0.7}$ & $\mathrm{BDBIM}_{p_{b}=0.35}$ & Gaussian \\
\hline 4 & $1.48231(6)$ & $1.46813(5)$ & $1.49036(8)$ & $1.48480(6)$ & $1.49164(9)$ & $1.49145(13)$ \\
5 & $1.48985(6)$ & $1.47597(6)$ & $1.49853(8)$ & & & $1.4996(2)$ \\
6 & $1.49446(6)$ & $1.48193(6)$ & $1.50300(9)$ & $1.49618(9)$ & $1.50788(9)$ & $1.5033(2)$ \\
7 & $1.49753(6)$ & $1.48642(6)$ & $1.50544(9)$ & & & \\
8 & $1.49987(6)$ & $1.48984(6)$ & $1.50714(9)$ & $1.50082(9)$ & $1.51320(13)$ & $1.5063(5)$ \\
9 & $1.50136(6)$ & $1.49260(6)$ & $1.50815(9)$ & & & \\
10 & $1.50273(6)$ & $1.49478(6)$ & $1.50889(9)$ & $1.50382(11)$ & $1.5146(2)$ & \\
11 & $1.50383(6)$ & $1.49665(6)$ & $1.50946(9)$ & & & \\
12 & $1.50469(6)$ & $1.49781(7)$ & $1.50984(13)$ & & & \\
13 & $1.50541(11)$ & & & & & \\
14 & $1.50618(10)$ & $1.50030(12)$ & $1.5103(3)$ & & & \\
16 & $1.50702(13)$ & $1.50220(13)$ & $1.5113(3)$ & & & \\
20 & $1.5081(3)$ & $1.5048(5)$ & & & & \\
24 & $1.5089(4)$ & & & & & \\
28 & $1.5108(13)$ & & & & & \\
\hline \hline
\end{tabular}

0.627(4) and 0.635(9) for an Ising model with bimodal and Gaussian distributed bonds, respectively. Thus, in most of the runs $\beta_{\max }$ was chosen so that $R_{\xi}\left(\beta_{\max }, L\right) \approx 0.63$. Only in the most recent simulations (two runs with $L=20$ and 24) of the $\pm J$ Ising model at $p=0.5$ did we take $\beta_{\max }$ such that $R_{\xi}\left(\beta_{\max }, L\right) \approx 0.66$. We checked thermalization by using the recipe outlined in Ref. 30, see App. B for some details.

As already emphasized in Refs. 46,69, in high-precision MC studies of random systems one should be careful when computing disorder averages of products of thermal expectations, for instance the quartic cumulant $U_{22}$ defined in Eq. (17). Indeed, naive estimators have a bias that may become significantly larger than the statistical error if $N_{s}$ is large. We use (essentially) bias-free estimators, defined as discussed in Ref. 46; some details are given in App. B.

In total, the MC simulations took approximately 40 years of CPU time on a single core of a $2.4 \mathrm{GHz}$ AMD Opteron processor.

\section{UNIVERSALITY AND CORRECTION-TO-SCALING EXPONENT $\omega$}

\section{A. Analysis of the quartic cumulants at fixed $R_{\xi}$}

In this section we investigate the universality of the critical behavior of Ising spin-glass models by comparing the limit for $L \rightarrow \infty$ of $U_{4}$ and $U_{22}$ computed at fixed $R_{\xi}$, denoted by $\bar{U}_{4}$ and $\bar{U}_{22}$, respectively. As discussed in Sec. III, for sufficiently large $L, \bar{U}_{4}$ and $\bar{U}_{22}$ are expected to behave as

$$
\bar{U}_{\#}=\bar{U}_{\#}^{*}+c_{\#} L^{-\omega}
$$


TABLE II: Quartic cumulant $\bar{U}_{22}$ at fixed $R_{\xi}=0.63$ for the $\pm J$ Ising model at $p=0.5$ and $p=0.7$, for the BDBIM at $p_{b}=0.45,0.7,0.35$, and for the Ising spin-glass model with Gaussian bond distribution.

\begin{tabular}{rlrlllc}
\hline \hline$L$ & \multicolumn{1}{c}{ $\pm J_{p=0.5}$} & $\pm J_{p=0.7}$ & $\mathrm{BDBIM}_{p_{b}=0.45}$ & $\mathrm{BDBIM}_{p_{b}=0.7}$ & $\mathrm{BDBIM}_{p_{b}=0.35}$ & Gaussian \\
\hline 4 & $0.13714(6)$ & $0.13955(6)$ & $0.13581(9)$ & $0.13602(7)$ & $0.14635(10)$ & $0.13522(15)$ \\
5 & $0.14088(7)$ & $0.14087(6)$ & $0.13935(10)$ & & & $0.14014(24)$ \\
6 & $0.14277(7)$ & $0.14181(7)$ & $0.14166(10)$ & $0.14193(10)$ & $0.14501(10)$ & $0.14227(24)$ \\
7 & $0.14392(6)$ & $0.14262(7)$ & $0.14308(10)$ & & & \\
8 & $0.14478(7)$ & $0.14318(7)$ & $0.14415(10)$ & $0.14414(10)$ & $0.14597(15)$ & $0.1434(5)$ \\
9 & $0.14522(7)$ & $0.14380(7)$ & $0.14470(10)$ & & & \\
10 & $0.14561(7)$ & $0.14418(7)$ & $0.14518(10)$ & $0.14536(12)$ & $0.14586(24)$ & \\
11 & $0.14595(7)$ & $0.14453(7)$ & $0.14566(10)$ & & & \\
12 & $0.14618(7)$ & $0.14465(7)$ & $0.14605(14)$ & & & \\
13 & $0.14650(11)$ & & & & & \\
14 & $0.14671(10)$ & $0.14531(13)$ & $0.1462(3)$ & & & \\
16 & $0.14675(14)$ & $0.14553(14)$ & $0.1469(4)$ & & & \\
20 & $0.1469(4)$ & $0.1458(5)$ & & & & \\
24 & $0.1477(5)$ & & & & & \\
28 & $0.1496(14)$ & & & & & \\
\hline \hline
\end{tabular}

where the constants $\bar{U}_{\#}^{*}$ are universal (model independent) but depend on the fixed value of $R_{\xi} ; \omega$ is the leading scaling-correction exponent.

In Tables I and II we report the estimates of $\bar{U}_{4}$ and $\bar{U}_{22}$ at fixed $R_{\xi}=0.63$ for different models. Without performing any analysis, one can immediately observe that the results obtained for the different models are very close, and appear to approach the same large- $L$ limit as $L$ increases. For instance, the estimates of $\bar{U}_{4}$ for the largest lattices differ at most by $0.5 \%$, while those of $\bar{U}_{22}$ vary by a few percent. This already provides strong support to universality.

For a more detailed analysis, let us first consider the three models for which we have most data, the $\pm J$ model at $p=0.5$ and $p=0.7$, and the BDBIM at $p_{b}=0.45$. The MC estimates of $\bar{U}_{4}(L)$ are shown in Fig. 2 versus $1 / L$. The results for the $\pm J$ Ising model at $p=0.5$ and $p=0.7$ fall quite nicely on two straight lines that approach the same point as $L \rightarrow \infty$. They support the universality of the critical behavior and show the presence of scaling corrections with an exponent $\omega \approx 1.0$. In the case of the BDBIM with $p_{b}=0.45$, the data apparently show a faster approach to the same infinite-volume limit. A fit of $\bar{U}_{4}(L)$ to $\bar{U}_{4}^{*}+c L^{-\varepsilon}$ using all data with $L>4$ gives an effective exponent $\varepsilon \approx 2$. However, for sufficiently large $L$, $L \gtrsim 10$ say, scaling corrections are consistent with a linear $1 / L$ behavior, as in the other models. The corresponding amplitude $\left|c_{4}\right|$ is quite small, at least a factor of two smaller than in the undiluted bimodal model. This means that for $L \lesssim 10$ next-to-leading corrections to scaling dominate and determine the apparent approach of the data to the infinite-volume limit. Since the ratios of the amplitudes of the leading scaling corrections are universal, a small $\left|c_{4}\right|$ implies that the leading nonanalytic scaling correction is small for any quantity. Thus, in this model the approach of any thermodynamic quantity to the critical limit should 


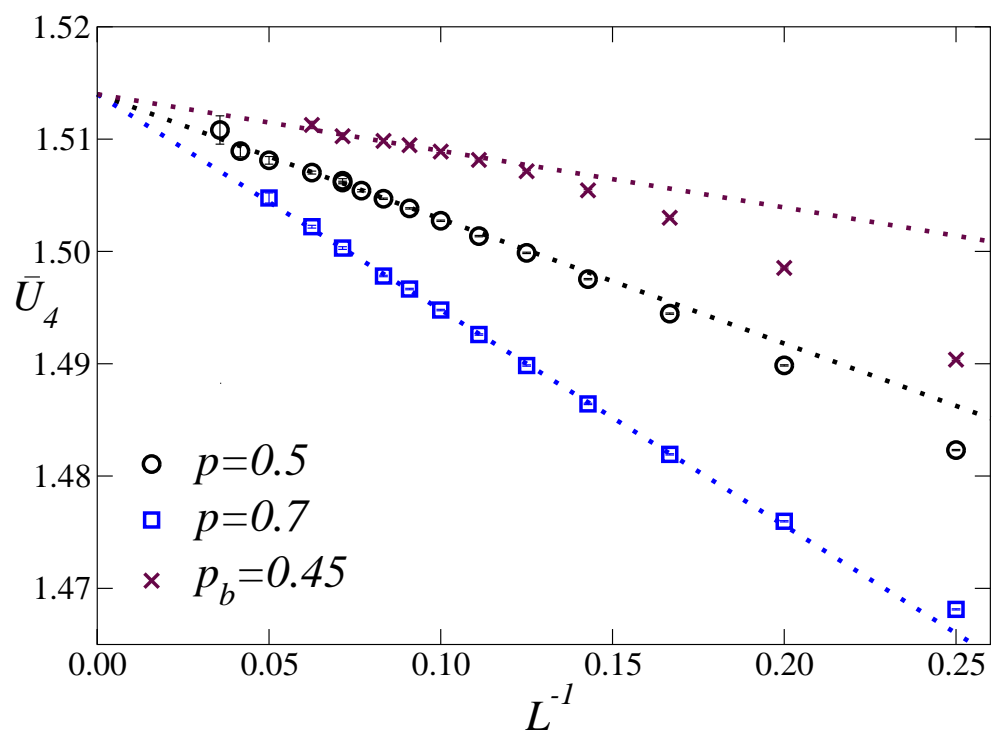

FIG. 2: (Color online) Estimates of $\bar{U}_{4}(L)$ vs $L^{-1}$, for the $\pm J$ model at $p=0.5$ and $p=0.7$, and the BDBIM at $p_{b}=0.45$. The dotted lines are drawn to guide the eye.

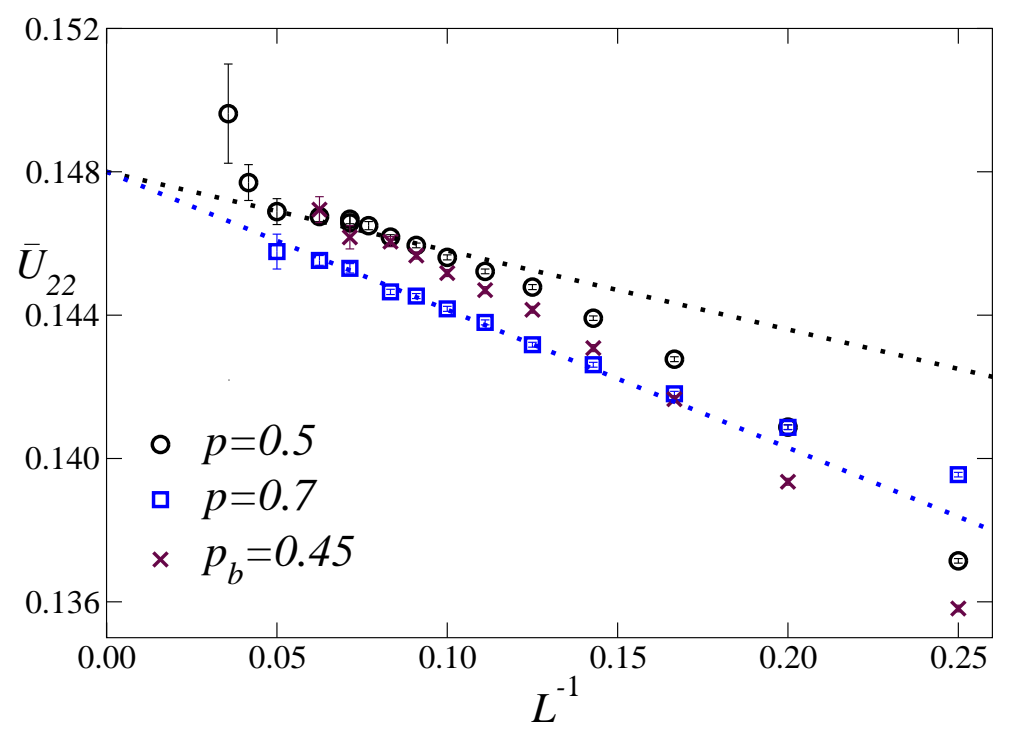

FIG. 3: (Color online) Estimates of $\bar{U}_{22}(L)$ vs $L^{-1}$, for the $\pm J$ model at $p=0.5$ and $p=0.7$, and the BDBIM at $p_{b}=0.45$. The dotted lines are drawn to guide the eye.

be faster than in the $\pm J$ models without dilution, as already noted in Ref. 28, at least for sufficiently large lattice sizes, where next-to-leading corrections can be neglected. Similar results are obtained for $\bar{U}_{22}$, see Fig. 3. However, in this case next-to-leading corrections appear to be more important, since the $1 / L$ behavior is observed for somewhat larger values of $L$.

In order to estimate the universal infinite-volume values $\bar{U}_{4}^{*}$ and $\bar{U}_{22}^{*}$, we perform fits of $\bar{U}_{\#}$ to $\bar{U}_{\#}^{*}+c_{\#} L^{-\varepsilon}$. The exponent $\varepsilon$ is either a free parameter, or is set equal to 1 , which corresponds, as we discuss below, to our best estimate of the leading scaling-correction 
TABLE III: Results of the fits of $\bar{U}_{4}$ and $\bar{U}_{22}$ at fixed $R_{\xi}=0.63$ to $\bar{U}^{*}+c L^{-\varepsilon}$ for several values of $L_{\text {min }}$, the minimum lattice size allowed in the fits. DOF is the number of degrees of freedom of the fit. The fits labelled " $p=0.5 \& p=0.7$ " are fits in which the data for $p=0.5$ and $p=0.7$ are analyzed together, assuming the universality of $\bar{U}_{4}^{*}$ and $\bar{U}_{22}^{*}$.

\begin{tabular}{|c|c|c|c|c|c|c|}
\hline Model & & $L_{\min }$ & $\bar{U}_{4}^{*}$ & $\chi^{2} / \mathrm{DOF}$ & $\bar{U}_{22}^{*}$ & $\chi^{2} / \mathrm{DOF}$ \\
\hline \multirow[t]{5}{*}{ $\pm J, p=0.5$} & free $\varepsilon$ & 6 & $1.5127(4)$ & 0.7 & $0.1478(3)$ & 0.7 \\
\hline & & 8 & $1.5135(9)$ & 0.8 & $0.1487(9)$ & 0.7 \\
\hline & & 10 & $1.5119(11)$ & 0.4 & $0.1482(12)$ & 0.8 \\
\hline & $\varepsilon=1$ & 8 & $1.5144(2)$ & 0.8 & $0.1490(2)$ & 0.6 \\
\hline & & 12 & $1.5139(4)$ & 0.6 & $0.1488(4)$ & 0.9 \\
\hline \multirow[t]{4}{*}{ $\pm J, p=0.7$} & free $\varepsilon$ & 6 & $1.5153(7)$ & 2.0 & $0.1481(8)$ & 0.8 \\
\hline & & 8 & $1.5145(14)$ & 2.7 & $0.1471(9)$ & 0.9 \\
\hline & $\varepsilon=1$ & 8 & $1.5143(2)$ & 2.3 & $0.1478(2)$ & 0.9 \\
\hline & & 12 & $1.5153(5)$ & 0.1 & $0.1482(5)$ & 0.8 \\
\hline \multirow[t]{4}{*}{$\mathrm{BDBIM}, p_{b}=0.45$} & free $\varepsilon$ & 6 & $1.5120(3)$ & 0.5 & $0.1479(6)$ & 0.6 \\
\hline & & 9 & $1.5132(22)$ & 0.6 & $0.1485(23)$ & 0.5 \\
\hline & $\varepsilon=1$ & 8 & $1.5153(3)$ & 0.8 & $0.1496(3)$ & 0.4 \\
\hline & & 12 & $1.5148(12)$ & 1.2 & $0.1489(13)$ & 1.0 \\
\hline \multirow[t]{6}{*}{ $\pm J, p=0.5 \& p=0.7$} & $\varepsilon=1$ & 8 & $1.5143(1)$ & 1.2 & $0.1485(1)$ & 2.1 \\
\hline & & 12 & $1.5145(3)$ & 0.9 & $0.1486(3)$ & 0.8 \\
\hline & & 16 & $1.5133(9)$ & 0.4 & $0.1489(10)$ & 1.1 \\
\hline & $\varepsilon=1.2$ & 8 & $1.5120(1)$ & 4.9 & $0.1479(1)$ & 2.5 \\
\hline & & 12 & $1.5127(3)$ & 0.4 & $0.1482(3)$ & 0.9 \\
\hline & & 16 & $1.5123(8)$ & 0.3 & $0.1486(9)$ & 1.1 \\
\hline
\end{tabular}

exponent $\omega$. In Table III we report the results. They are all consistent with the estimates

$$
\bar{U}_{4}^{*}=1.514(2), \quad \bar{U}_{22}^{*}=0.148(1) .
$$

The accuracy and stability of the fits can also be appreciated in Fig. 4, where the fit results are plotted versus the minimum lattice size $L_{\min }$ allowed in the fits. We can thus conclude that the estimates of the quartic cumulants for the $\pm J$ Ising model and the BDBIM at $p_{b}=0.45$ are fully consistent with universality. The relative differences are approximately one per mille in the case of $\bar{U}_{4}$ and one per cent for $\bar{U}_{22}$.

We also considered the BDBIM for the other dilution values, i.e., for $p_{b}=0.7$ and $p_{b}=$ 0.35 , though in this case we have data only up to $L=10$. The estimates of $\bar{U}_{4}$ are shown versus $1 / L$ in Fig. 5 . For $p_{b}=0.7$ scaling corrections clearly behave as $1 / L$ and are larger than at $p_{b}=0.45$. For the scaling-correction amplitude $c_{4}$ defined in Eq. (47) we obtain the estimate $c_{4} \approx-0.10$, to be compared with the result $c_{4} \approx-0.11$ for the $\pm J$ model at $p=0.5$ and $c_{4} \approx-0.05$ for the diluted model at $p_{b}=0.45$. For $p_{b}=0.35$ we do not have enough data to estimate $c_{4}$; however, the data reported in Fig. 5 apparently approach the infinite-volume limit faster than at $p_{b}=0.45$. As indicated by the $\mathrm{MC}$ data for $p_{b} \lesssim 0.27$ of Ref. 63, the scaling corrections should increase as $p_{b}$ further decreases. This suggests that 

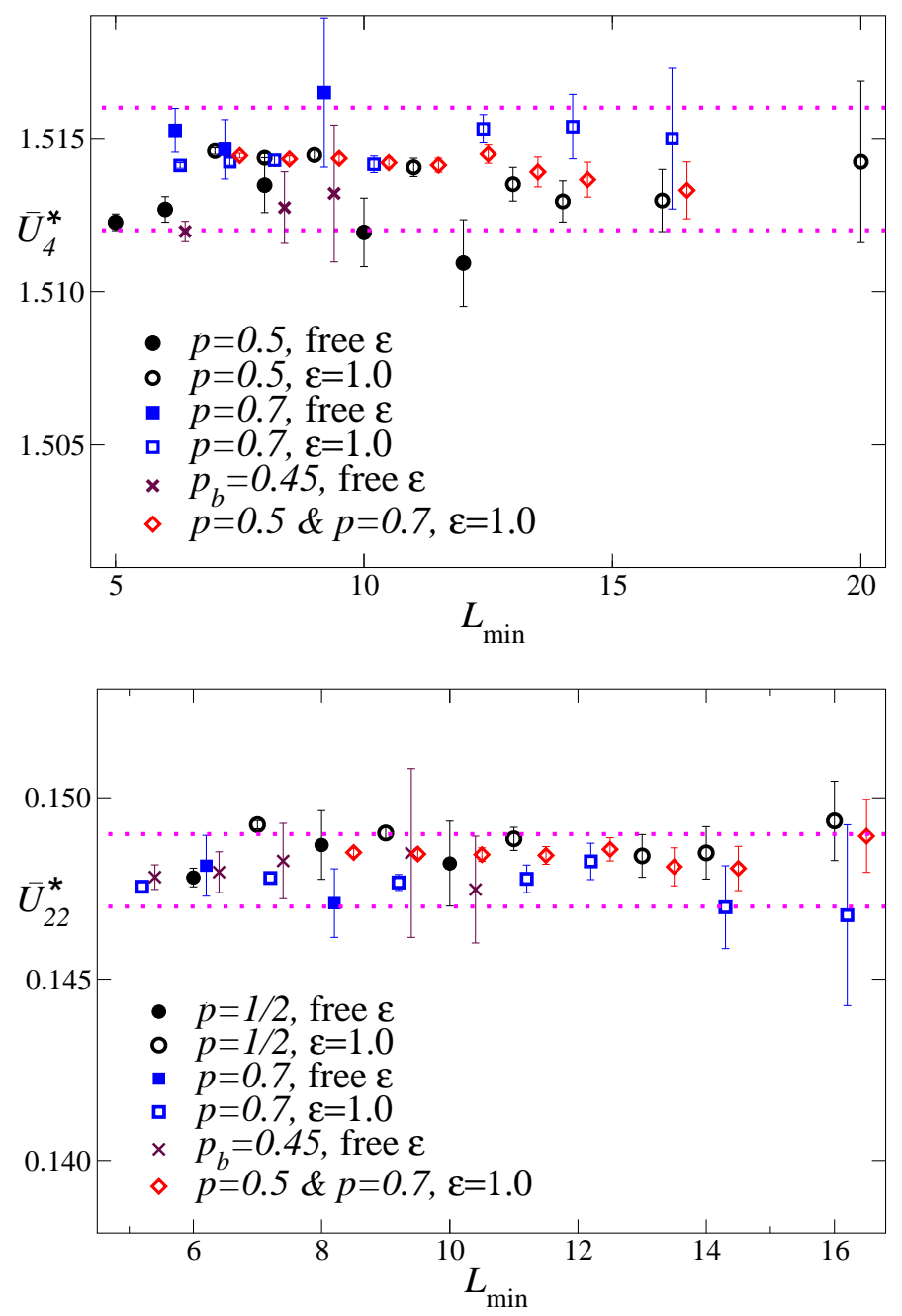

FIG. 4: Estimates of $\bar{U}_{4}^{*}$ and $\bar{U}_{22}^{*}$, obtained by fitting the quartic cumulants $\bar{U}_{\#}$ at fixed $R_{\xi}=0.63$ to $\bar{U}_{\#}^{*}+c L^{-\varepsilon}$. Here $L_{\text {min }}$ is the minimum lattice size allowed in the fits. The fits labelled " $p=0.5$ $\& p=0.7 "$ are fits in which the data for $p=0.5$ and $p=0.7$ are analyzed together. The dotted lines correspond to the final estimates $\bar{U}_{4}^{*}=1.514(2)$ and $\bar{U}_{22}^{*}=0.148(1)$.

the optimal model - the one with the smallest leading scaling corrections - corresponds to a dilution in the range $0.3 \lesssim p_{b}^{*} \lesssim 0.4$ : the model with $p_{b}=0.35$ should be close to the optimal one.

The estimates of $\bar{U}_{4}$ and $\bar{U}_{22}$ for the Ising spin-glass model with Gaussian bond distribution are shown in Figs. 5 and 6: they are very close, and clearly approach, the estimates (48). The results for $L=8$ differ by $0.5 \%\left(\bar{U}_{4}\right)$ and $3 \%\left(\bar{U}_{22}\right)$ from the asymptotic value.

In conclusion, the results for the $\pm J$ model, for the BDBIM, and for the model with Gaussian distributed couplings provide a very strong evidence of universality for the critical behavior of Ising spin-glass models along the PG transition line. 


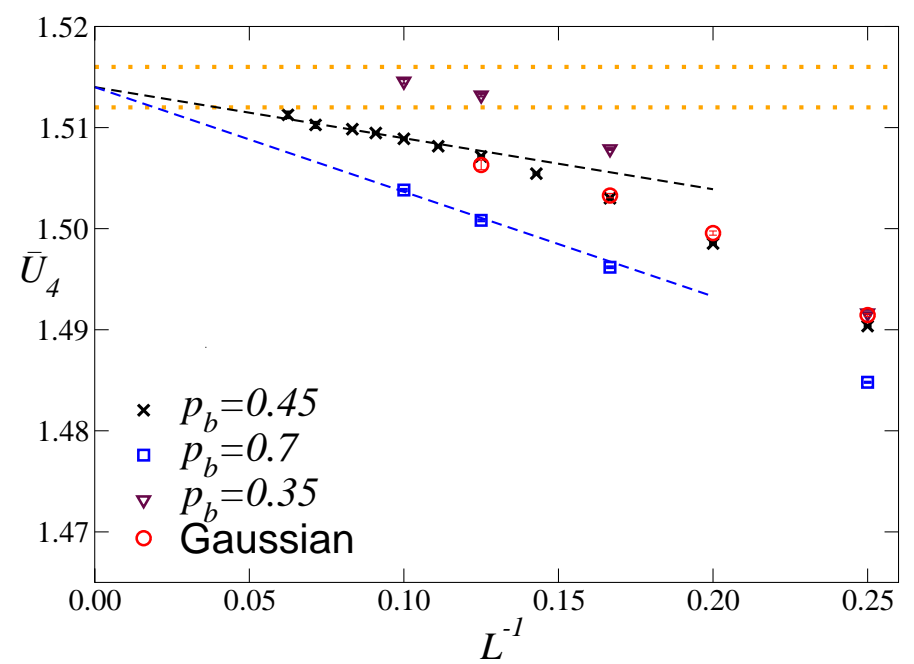

FIG. 5: Estimates of $\bar{U}_{4}(L)$ for the BDBIM for several values of $p_{b}$ and for the Ising spin-glass model with Gaussian bond distribution. The dotted lines correspond to the estimate $(48), \bar{U}_{4}^{*}=1.514(2)$. The dashed straight lines associated with the data at $p_{b}=0.7$ and $p_{b}=0.45$ show the linear behavior of the data for their largest lattices.

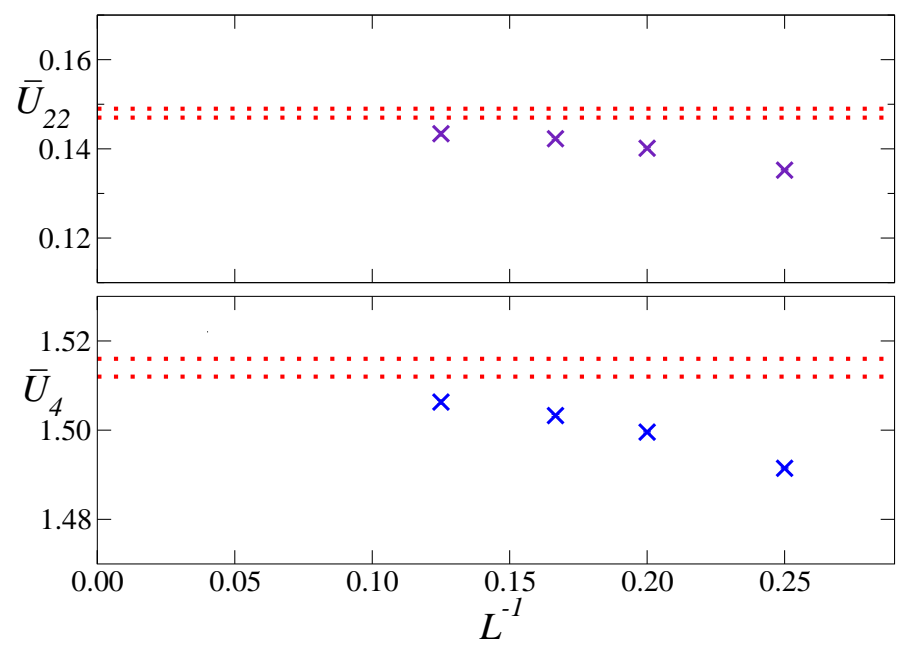

FIG. 6: $\bar{U}_{4}$ (below) and $\bar{U}_{22}$ (above) for the Ising spin-glass model with Gaussian bond distribution. The dotted lines correspond to the estimates (48), $\bar{U}_{4}^{*}=1.514(2)$ and $\bar{U}_{22}^{*}=0.148(1)$.

\section{B. The RG exponent of the leading irrelevant operator}

The analyses of $\bar{U}_{4}$ and $\bar{U}_{22}$ also give estimates of $\omega$. The most precise ones are obtained from fits of $\bar{U}_{4}$. In Fig. 7 we show the estimates of $\omega$ obtained from fits of $\bar{U}_{4}$ to $\bar{U}_{4}^{*}+c_{p} L^{-\omega}$ and from fits of the difference $\bar{U}_{4}(p=0.5 ; L)-\bar{U}_{4}(p=0.7 ; L)$ to $b L^{-\omega}$. We estimate

$$
\omega=1.0(1)
$$

Consistent but less precise results are obtained from fits of $\bar{U}_{22}$. The result (49) is consistent with the less precise estimates $\omega=0.84_{-0.37}^{+0.43}$ and $\omega=1.0(4)$ reported in Refs. 21 and 27, 


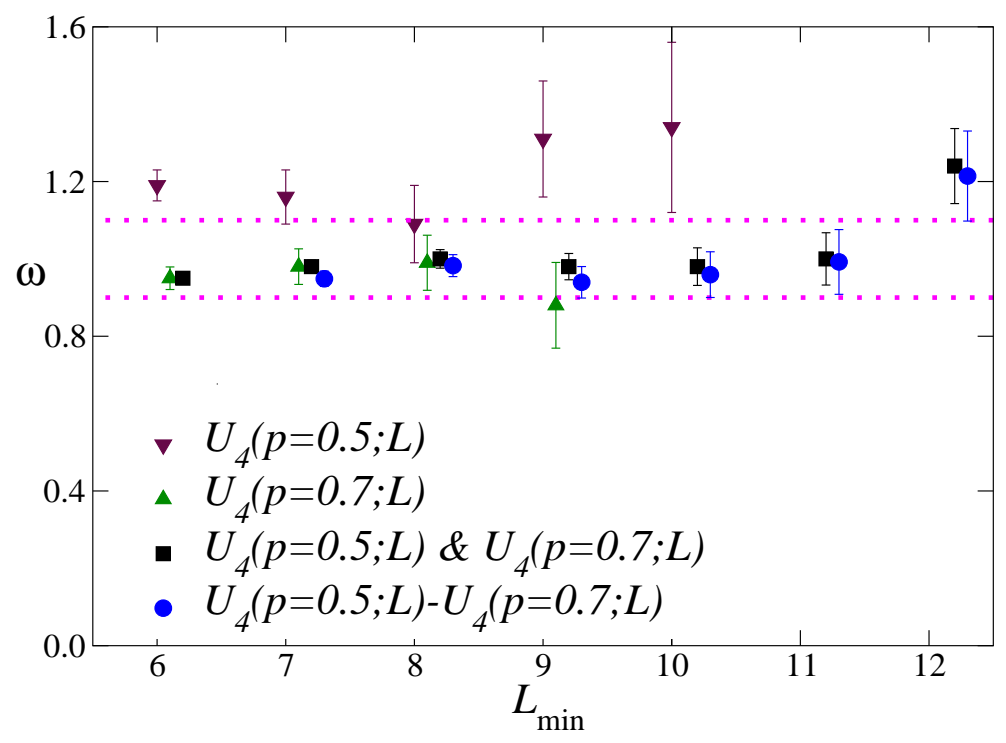

FIG. 7: (Color online) Estimates of the leading correction-to-scaling exponent $\omega$ versus the minimum lattice size $L_{\min }$ used in the fit. The fits labelled " $p=0.5 \& p=0.7$ " are fits in which the data for $p=0.5$ and $p=0.7$ are analyzed together. The dotted lines correspond to the final estimate $\omega=1.0(1)$.

respectively.

In order to verify that scaling corrections are controlled by a single correction-to-scaling term with exponent $\omega=1$ and we have not determined an effective exponent arising from several almost degenerate exponents, we check that the ratio $c_{22} / c_{4}$ is universal, where $c_{\#}$ is the scaling-correction amplitude appearing in Eq. (47), as predicted by standard RG arguments. In order to determine this ratio, we fit together the available estimates of $\bar{U}(L)$ for the $\pm J$ Ising models at $p=0.5$ and $p=0.7$ to $\bar{U}(L)=\bar{U}^{*}+c_{p} L^{-\varepsilon}$, fixing $\varepsilon=1$ and taking $\bar{U}^{*}$ and the two amplitudes $c_{p=0.5}$ and $c_{p=0.7}$ as free parameters. Fits of the data for $L \geq L_{\min }=10$ give

$$
\bar{U}_{4}^{*}=1.5142(2), \quad c_{4}(p=0.5)=-0.114(2), \quad c_{4}(p=0.7)=-0.194(2),
$$

$\left(\chi^{2} / \mathrm{DOF} \approx 1.3\right.$, where DOF is the number of degrees of freedom of the fit $)$, and

$$
\bar{U}_{22}^{*}=0.1484(2), \quad c_{22}(p=0.5)=-0.027(2), \quad c_{22}(p=0.7)=-0.044(2),
$$

$\left(\chi^{2} / \mathrm{DOF} \approx 1.4\right)$. It follows

$$
\begin{aligned}
& c_{22} / c_{4}=0.24(2) \quad \text { for } \quad p=0.5, \\
& c_{22} / c_{4}=0.23(1) \quad \text { for } \quad p=0.7,
\end{aligned}
$$

which are in good agreement. These results are quite stable with increasing $L_{\min }$. For $L_{\text {min }}=12$ we obtain $c_{22} / c_{4}=0.24(3)$ and $c_{22} / c_{4}=0.24(2)$ for $p=0.5$ and $p=0.7$, while for $L_{\text {min }}=14$ we find $c_{22} / c_{4}=0.19(9)$ and $c_{22} / c_{4}=0.21(5)$, respectively; in both cases the fits have $\chi^{2} / \mathrm{DOF} \lesssim 1$. Moreover, the variation of the ratio $c_{22} / c_{4}$ with respect to a change of the exponent $\varepsilon$ by \pm 0.1 [it corresponds to the uncertainty of $\omega, \omega=1.0(1)$ ], is smaller than the above-reported errors. These results fully support our interpretation of the $O\left(L^{-1.0}\right)$ corrections as the corrections arising from the leading irrelevant scaling field. 


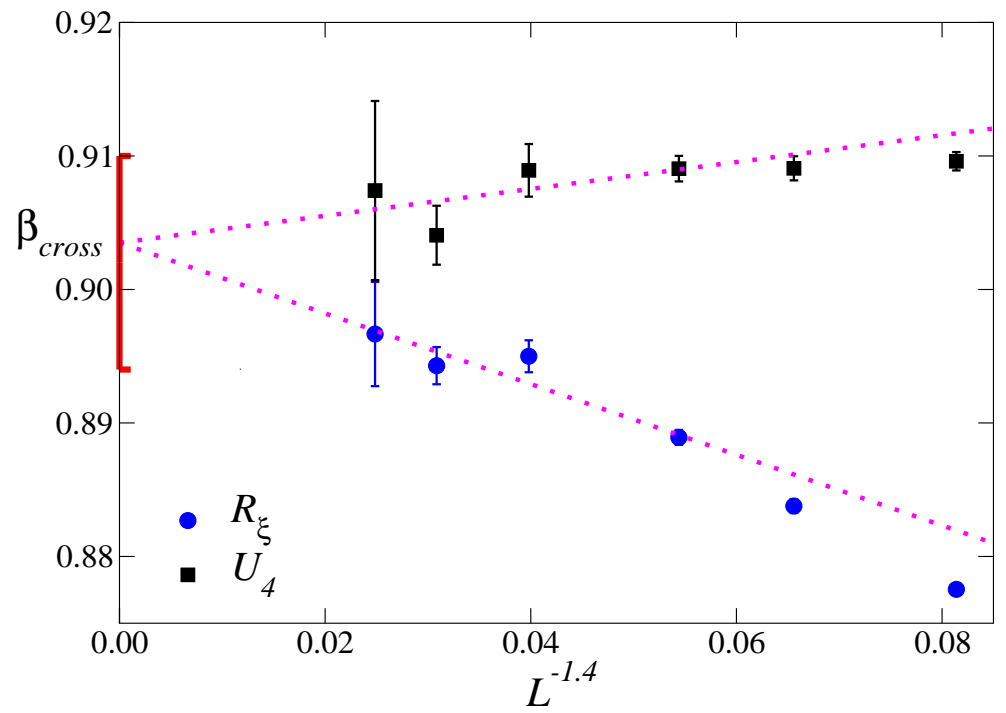

FIG. 8: (Color online) Crossing point $\beta_{\text {cross }}(L)$ for the $\pm J$ Ising model at $p=0.5$, from several pairs $L, 2 L$, as obtained from $R_{\xi}$ and $U_{4}$, versus $L^{-1 / \nu-\omega} \sim L^{-1.4}$. The dotted lines show the result of a fit to $\beta_{c}+c L^{-1.4}$, which takes into account only the data satisfying $L \geq L_{\min }=8$-it corresponds to $L_{\text {min }}^{-1.4} \approx 0.054$. The bar along the $y$-axis corresponds to the final estimate $\beta_{c}=0.902(8)$.

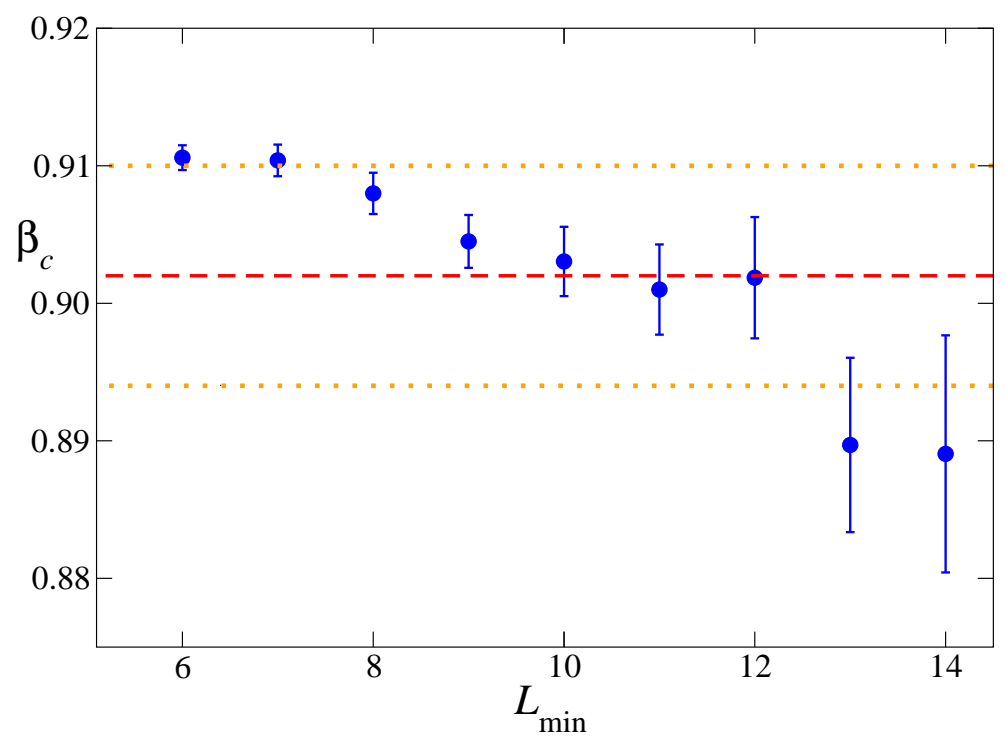

FIG. 9: (Color online) Estimates of $\beta_{c}$ as obtained from fits of $R_{\xi}$ and $U_{4}$ to Eq. (56) with $\omega=1$, for the $\pm J$ Ising model at $p=0.5$. All fits have $\chi^{2} / \mathrm{DOF}<1.5$. The lines correspond to the final estimate $\beta_{c}=0.902(8)$. 


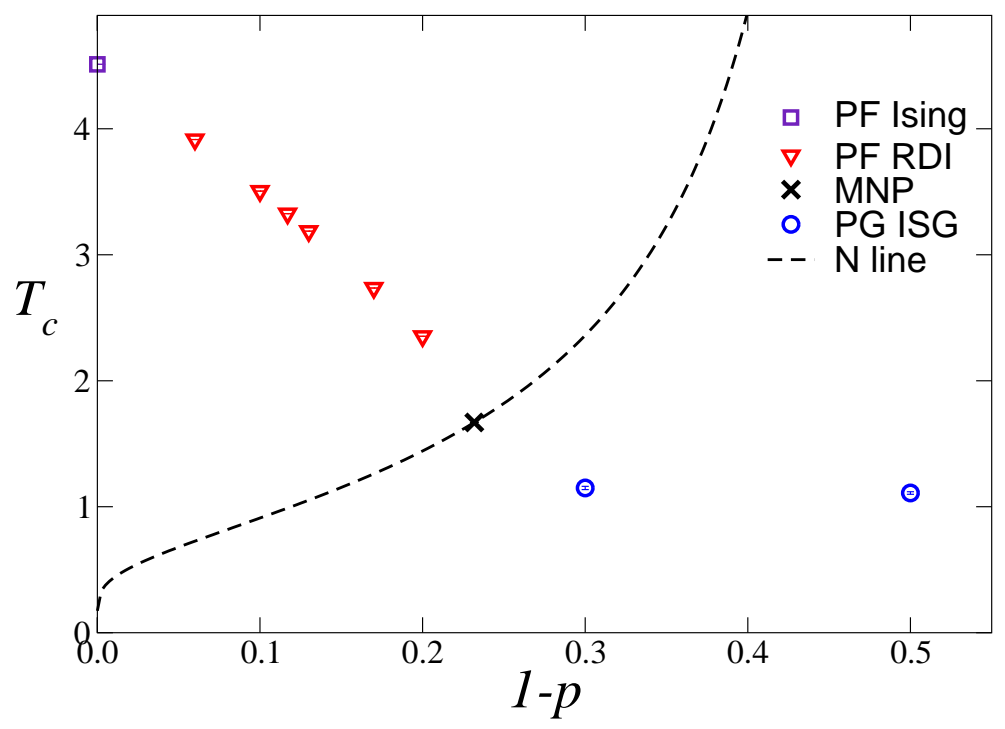

FIG. 10: (Color online) The values of the critical temperature $T_{c}$ versus $1-p$ for the various transitions of the $\pm J$ Ising model. The estimates at the paramagnetic-ferromagnetic transitions are taken from Ref. 43 for the Ising transition, from Ref. 44 for the RDI transition, and from Ref. 42 for that along the $N$ line.

\section{CRITICAL TEMPERATURE AND EXPONENTS}

\section{A. Estimates of $\beta_{c}$}

In order to determine the critical temperature, we perform a standard FSS analysis of $R_{\xi}$ and the quartic cumulants. Figure 8 shows the crossing points $\beta_{\text {cross }}(L)$ determined by solving the equation

$$
R\left(\beta_{\text {cross }}, L\right)=R\left(\beta_{\text {cross }}, 2 L\right),
$$

for the $\pm J$ Ising model at $p=0.5$, using $R_{\xi}$ and $U_{4}$. In the large- $L$ limit $\beta_{\text {cross }}(L)$ is expected to converge to $\beta_{c}$ as

$$
\beta_{\text {cross }}(L)-\beta_{c} \sim L^{-1 / \nu-\omega} \sim L^{-1.4},
$$

since $\nu \approx 2.45$ and $\omega \approx 1.0$. We perform a combined fit of $R_{\xi}$ and $U_{4}$ to $\beta_{c}+c_{\#} L^{-\varepsilon}$ with $\varepsilon=-1.4$, taking $\beta_{c}$ and the two amplitudes $c_{R_{\xi}}$ and $c_{U_{4}}$ as free parameters. Using only the results with $L \geq L_{\min }=8$, we obtain $\beta_{c}=0.9035(22)$ with $\chi^{2} / \mathrm{DOF} \approx 1.1$ (since the estimates of $R_{\xi}$ and $U_{4}$ are correlated the error is only indicative). The corresponding lines are drawn in Fig. 8. We also consider larger values of $L_{\min }$. We find that the estimates of $\beta_{c}$ vary significantly (much more than the statistical error) when $L_{\text {min }}$ varies, indicating that the systematic error due to the additional scaling corrections is significantly larger than the statistical one. Equivalently, one can perform fits to

$$
R\left(L, \beta_{c}\right)=R^{*}+b L^{-\omega},
$$

taking $R=R_{\xi}$ or $U_{4} \cdot{ }^{70}$ The results are shown in Fig. 9. Taking into account the dependence of the results on $L_{\min }$ and their variation as $\omega$ varies by one error bar we obtain the final estimates

$$
\beta_{c}=0.902(8), \quad T_{c}=1.109(10) .
$$




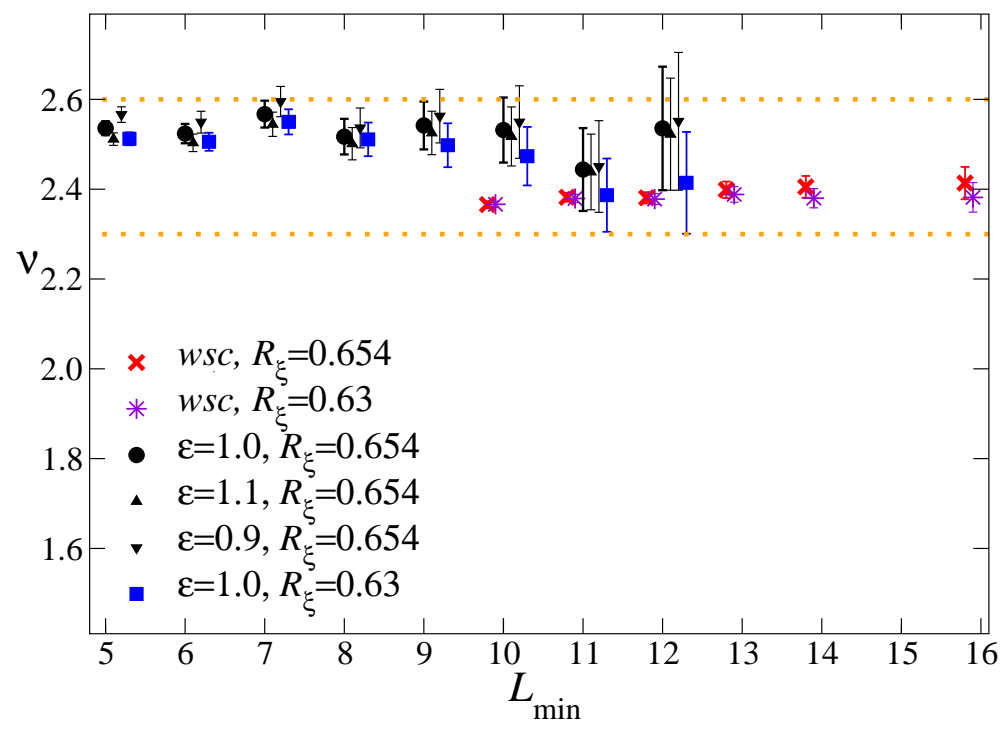

FIG. 11: (Color online) Estimates of the exponent $\nu$ from the analysis of $\bar{R}_{\xi}^{\prime}$, at $R_{\xi}=0.63$ and $R_{\xi}=0.654$, for the $\pm J$ Ising model at $p=0.5$, obtained by fitting the data without scaling corrections (wsc), Eq. (59), and with scaling corrections, Eq. (60). We only show the results of the fits which satisfy $\chi^{2} / \mathrm{DOF} \lesssim 1.5$, up to values of $L_{\text {min }}$ where the errors blow up. The dotted lines correspond to the final estimate $\nu=2.45(15)$.

We regard the error as conservative. We also consider the pseudocritical $\beta_{f}(L)$ defined in Sec. III at fixed $R_{\xi}$, which converges to $\beta_{c}$ as $L \rightarrow \infty$, cf. Eqs. (38) and (39). The results are consistent with the estimate (57). The analysis of the crossing points and the fits to Eq. (56) also provide estimates of the limit $L \rightarrow \infty$ of $R_{\xi}$ and $U_{4}$ at the critical point. We obtain

$$
R_{\xi}^{*}=0.645(15), \quad U_{4}^{*}=1.50(2) .
$$

Concerning the other models, similar analyses of $R_{\xi}$ and $U_{4}$ give $\beta_{c}=0.87(1)\left[T_{c}=\right.$ $1.149(13)]$ for the $\pm J$ model at $p=0.7$, and $\beta_{c}=1.54(2)\left[T_{c}=0.649(8)\right]$ for the BDBIM at $p_{b}=0.45$. The corresponding values of $R_{\xi}^{*}$ and $U_{4}^{*}$ are consistent with the estimates (58).

Finally, in Fig. 10 we collect all results for the critical temperature $T_{c}$ of the $\pm J$ Ising model at its various $\mathrm{PF}$ and $\mathrm{PG}$ transitions as a function of the disorder parameter $p$.

\section{B. The critical exponent $\nu$}

We estimate the critical exponent $\nu$ from the finite-size behavior of $R_{\xi}^{\prime} \equiv d R_{\xi} / d \beta$ and $U_{4}^{\prime} \equiv d U_{4} / d \beta$ at a fixed value $R_{\xi, f}$ of $R_{\xi}$. As we discussed in Sec. III, the best choice for $R_{\xi, f}$ is $R_{\xi}^{*}$, otherwise $\bar{R}_{\xi}^{\prime}$ and $U_{4}^{\prime}$ present analytic corrections. In the present case, the estimate $(58)$ of $R_{\xi}^{*}$ is not very precise, hence the corrections of order $L^{-1 / \nu} \sim L^{-0.4}$ cannot be completely neglected. However, since their amplitude is proportional to $\left(R_{\xi}-R_{\xi}^{*}\right)$, we can assume that they are small for $R_{\xi, f}$ close to $R_{\xi}^{*}$. Thus, in order to estimate the systematic error induced by them, we proceed as follows. We repeat the analysis of $\bar{R}^{\prime}$ at fixed $R_{\xi}$ using two different values of $R_{\xi}$ and neglecting in both cases the $L^{-1 / \nu}$ corrections: we use $R_{\xi}=0.630$ and $R_{\xi}=0.654$, which are close to our best estimate $R_{\xi}^{*}=0.645(15)$. For 


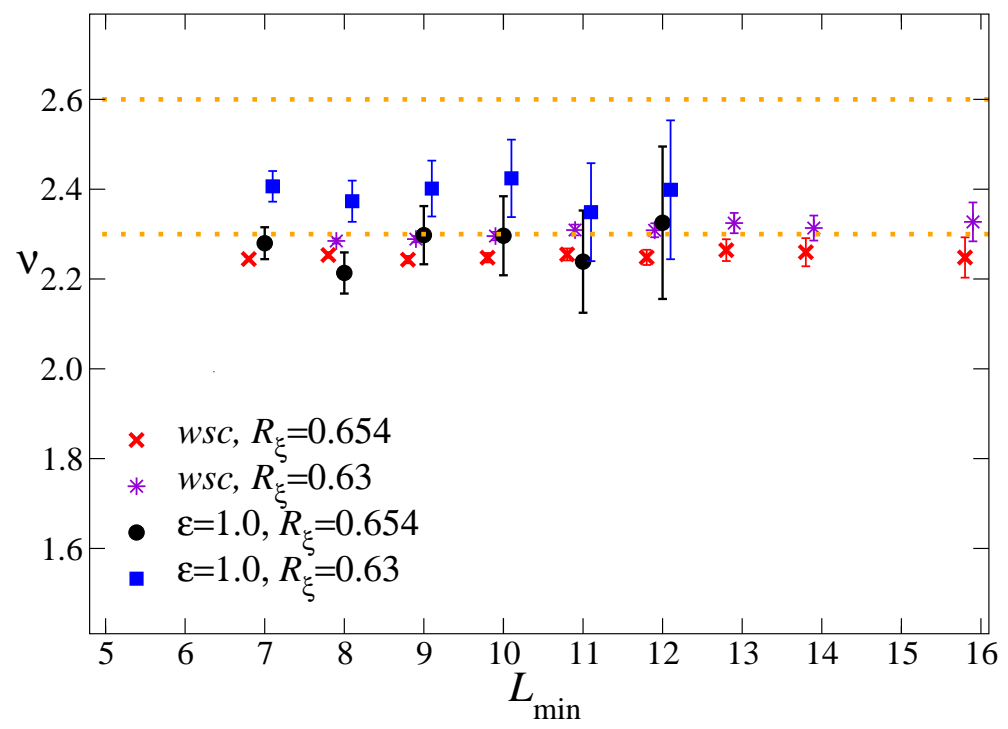

FIG. 12: (Color online) Estimates of the exponent $\nu$ from the analysis of $\bar{U}_{4}^{\prime}$, at $R_{\xi}=0.63$ and $R_{\xi}=0.654$, for the $\pm J$ Ising model at $p=0.5$, obtained by fits without scaling corrections (wsc), Eq. (59), and fits with scaling corrections, Eq. (60). We only show the results of the fits which satisfy $\chi^{2} / \mathrm{DOF} \lesssim 1.5$, up to values of $L_{\min }$ where the errors blow up. The dotted lines correspond to $\nu=2.45(15)$.

both values of $R_{\xi}$ we determine an estimate of $\nu$. Our final result is obtained by linear interpolation to $R_{\xi}=0.645$. The systematic error is estimated from the difference of the results at $R_{\xi}=0.630$ and $R_{\xi}=0.645$.

The exponent $\nu$ is obtained from fits of $\bar{R}_{\xi}^{\prime}$ and $\bar{U}_{4}^{\prime}$ to

$$
\begin{aligned}
& \ln \bar{R}^{\prime}=a+\frac{1}{\nu} \ln L, \\
& \ln \bar{R}^{\prime}=a+\frac{1}{\nu} \ln L+b L^{-\epsilon},
\end{aligned}
$$

with $\epsilon=\omega=1.0(1)$. The results of the fits of $\bar{R}_{\xi}^{\prime}$ for the $\pm J$ Ising model with $p=0.5$ are shown in Fig. 11 as a function of the minimum lattice size $L_{\min }$ allowed in the fits. They are quite stable with respect to $L_{\min }$, depend very little on the fixed value $R_{\xi}$, and change only slightly as $\omega$ varies by \pm 0.1 , corresponding to one error bar. In particular, the fit of $\bar{R}_{\xi}^{\prime}$ at $R_{\xi}=0.654$ to Eq. (60) gives $\nu=2.52(4)[2](\nu=2.53(7)[1])$ for $L \geq L_{\text {min }}=8$ ( $\left.L_{\text {min }}=10\right)$; here the error in brackets takes into account the uncertainty on $\omega$. Analogously, the fit of the data at $R_{\xi}=0.630$ gives $\nu=2.51(4)[2](\nu=2.47(7)[1])$ for the same values of $L_{\min }$. In both cases $\chi^{2} / \mathrm{DOF} \approx 1.1\left(\chi^{2} / \mathrm{DOF} \approx 1.3\right)$. Therefore, we obtain $\nu=2.52(6)$ and $2.51(8)$ at $R_{\xi}=0.645$ for $L_{\min }=8,10$. The systematic error due to the analytic corrections is small, 0.01 and 0.04 for the two values of $L_{\text {min }}$. The results from fits of $\bar{U}_{4}^{\prime}$, which are shown in Fig. 12, favor a smaller value of $\nu$, although in substantial agreement. Indeed, fits with $L_{\text {min }}=10$ give $\nu=2.30(9)$ at $R_{\xi}=0.654$ and $\nu=2.42(9)$ at $R_{\xi}=0.630$, thus suggesting the estimate $\nu=2.35(9)$. The error due to the analytic corrections is apparently larger than that obtained in the analysis of $\bar{R}_{\xi}^{\prime}$, approximately 0.07 . As our final result we take

$$
\nu=2.45(15),
$$




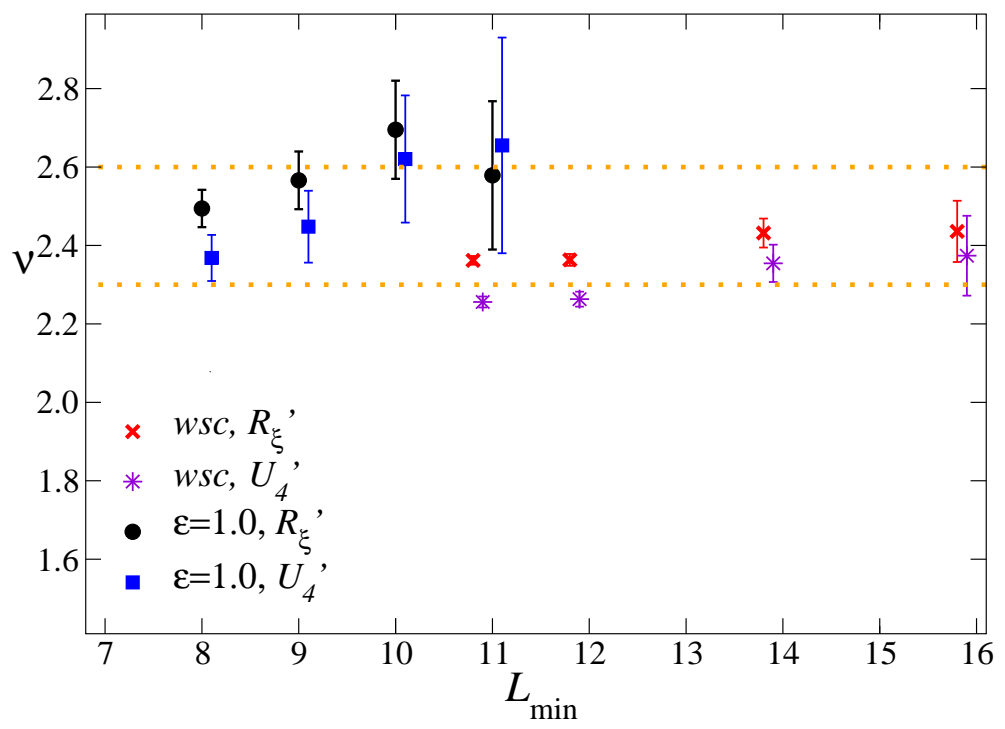

FIG. 13: (Color online) Estimates of the exponent $\nu$ from the analysis of $\bar{R}_{\xi}^{\prime}$ and $\bar{U}_{4}^{\prime}$, at $R_{\xi}=0.63$ for the $\pm J$ Ising model at $p=0.7$, obtained by fitting the data without scaling corrections (wsc) and with scaling corrections, Eq. (60). We only show the results of the fits with $\chi^{2} / \mathrm{DOF} \lesssim 1.5$, up to values of $L_{\min }$ where the errors blow up. The dotted lines correspond to $\nu=2.45(15)$.

which is consistent with the results obtained for $\bar{U}_{4}^{\prime}$ and $\bar{R}_{\xi}^{\prime}$.

Results at $R_{\xi}=0.63$ for the $\pm J$ model at $p=0.7$ and the BDBIM at $p_{b}=0.45$, shown in Figs. 13 and 14, respectively, are fully consistent with the estimate (61). For the $\pm J$ model at $p=0.7$, fits with $\varepsilon=1.0$ and $L_{\text {min }}=8$ of $\bar{R}_{\xi}^{\prime}$ and $\bar{U}_{4}^{\prime}$ at fixed $R_{\xi}=0.63$ give $\nu=2.49(5)$ and $\nu=2.37(6)$, respectively. In the case of the BDBIM at $p_{b}=0.45$ and again for $R_{\xi}=0.63$, fits without scaling corrections are in full agreement. Fits of $\bar{R}_{\xi}^{\prime}$ to (60) assuming $\varepsilon=2.0$ are also consistent: for $L_{\min }=7$ they give $\nu=2.52(4)$. On the other hand, the fits of $\bar{U}_{4}^{\prime}$ with $\varepsilon=2.0$ give results that are systematically larger; for instance, we obtain $\nu=2.61(5)$ for $L_{\min }=7$. This slight discrepancy is probably due to the fact that scaling corrections with exponent $\omega=1$ are small in the BDBIM at $p_{b}=0.45$ but not completely negligible: thus, the fits assume that corrections vanish faster than they really do, obtaining a slightly incorrect asymptotic estimate.

\section{The critical exponent $\eta$}

We estimate the exponent $\eta$ by analyzing the susceptibility $\chi$ at fixed $R_{\xi}$. Its critical behavior is discussed in Sec. III: at fixed $R_{\xi}$, it behaves as

$$
\bar{\chi}\left(L, R_{\xi}\right)=a L^{2-\eta}\left[1+a_{a}\left(R_{\xi}-R_{\xi}^{*}\right) L^{-1 / \nu}+\cdots+a_{\omega} L^{-\omega}+\ldots+a_{b} L^{-2+\eta}+\cdots\right],
$$

where the $O\left(L^{-2+\eta}\right)$ term is the background contribution, cf. Eq. (29). Since $R_{\xi}$ is not exactly equal to $R_{\xi}^{*}$, we must take into account the $O\left(L^{-1 / \nu}\right)$ corrections. As for $\nu$, the systematic error they induce is estimated by comparing the results at $R_{\xi}=0.630$ and $R_{\xi}=0.654$, which are close to the best estimate $R_{\xi}^{*}=0.645(15)$. We perform fits with and without scaling 


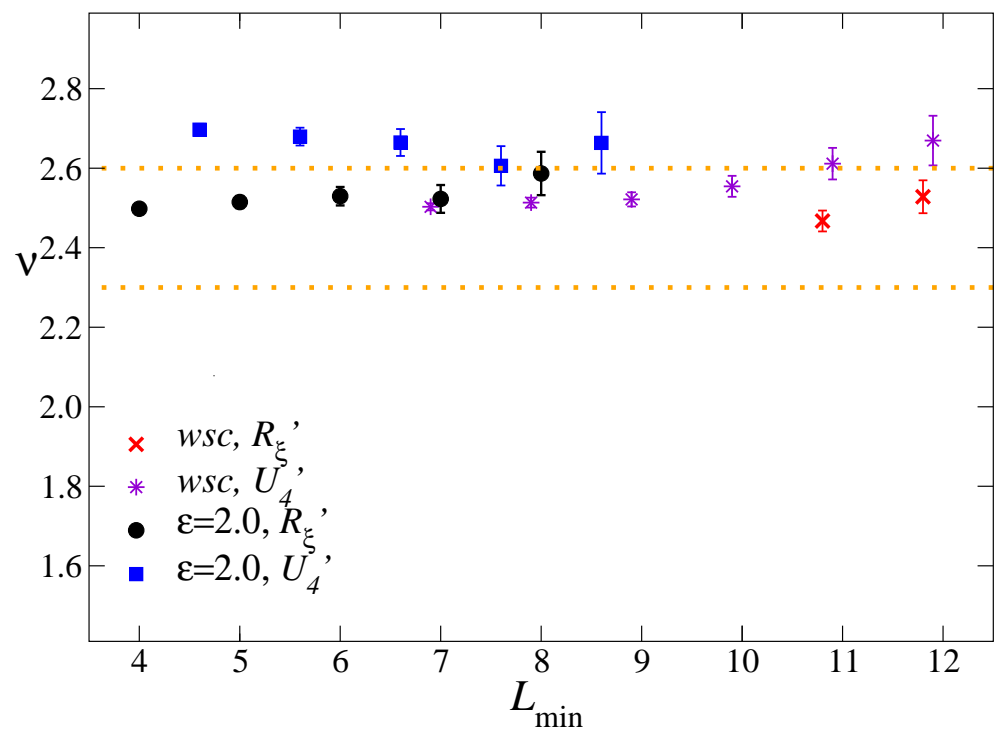

FIG. 14: (Color online) Estimates of the exponent $\nu$ from the analysis of $\bar{R}_{\xi}^{\prime}$ and $\bar{U}_{4}^{\prime}$, at $R_{\xi}=0.63$ for the BDBIM at $p_{b}=0.45$, obtained by fitting the data without scaling corrections (wsc) and with scaling corrections, Eq. (60). We only show the results of the fits with reasonable values of $\chi^{2} / \mathrm{DOF}$, up to values of $L_{\min }$ where the errors blow up. The dotted lines correspond to $\nu=2.45(15)$.

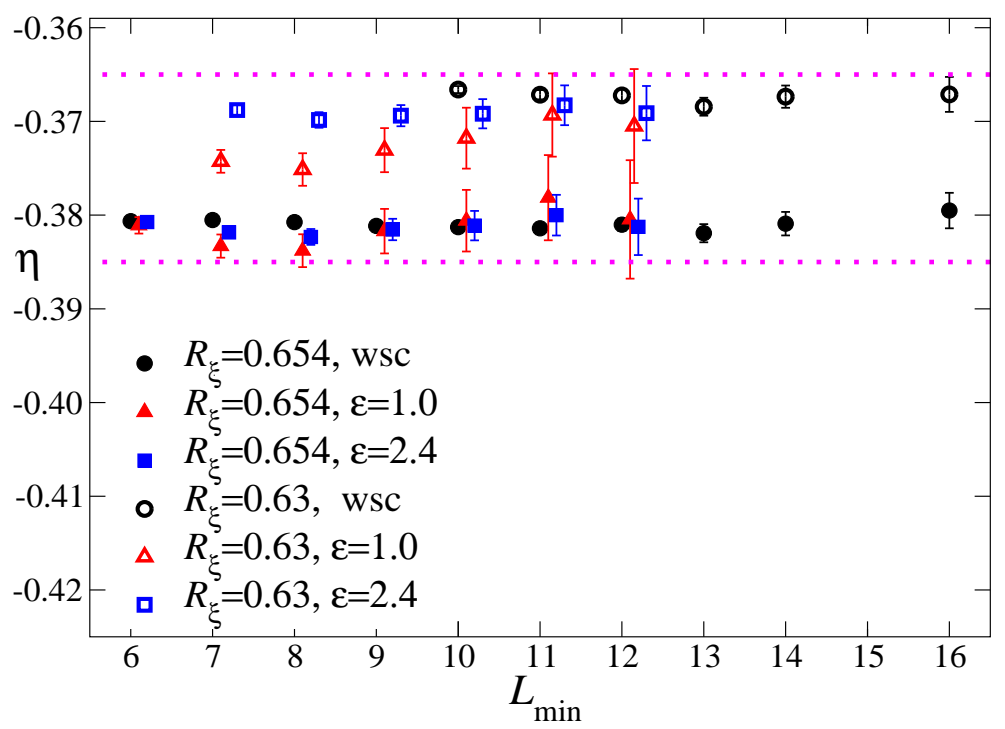

FIG. 15: (Color online) Estimates of the exponent $\eta$ from the analysis of $\bar{\chi}\left(L, R_{\xi}\right)$ for $R_{\xi}=0.63$ and $R_{\xi}=0.654$, for the $\pm J$ Ising model at $p=0.5$. Only results of fits with $\chi^{2} / \mathrm{DOF}<1.5$ are shown. The dotted lines correspond to the estimate $\eta=-0.375(10)$.

corrections, i.e. to

$$
\begin{aligned}
& \ln \chi=c+(2-\eta) \ln L, \\
& \ln \chi=c+(2-\eta) \ln L+c_{\varepsilon} L^{-\varepsilon},
\end{aligned}
$$


with $\varepsilon=1$ (the leading scaling correction arising from irrelevant scaling fields) and $\varepsilon=$ $2.4 \approx 2-\eta$ (to check the relevance of the background term, which might be large for small lattices) at $R_{\xi}=0.63$ and $R_{\xi}=0.654$. Fig. 15 shows the estimates of $\eta$ for the $\pm J$ model at $p=0.5$. As already mentioned, the systematic error due to the neglected $L^{-1 / \nu}$ corrections is estimated from the difference of the estimates at $R_{\xi}=0.63$ and $R_{\xi}=0.654$. Following the same method used to determine $\nu$, we obtain $\eta=-0.375(2)\{8\}$, where the first error is the statistical one while the second error takes into account the systematic error due to the residual $O\left(L^{-1 / \nu}\right)$ corrections and corresponds to the uncertainty on our estimate of $R_{\xi}^{*}$. Slightly smaller but perfectly consistent results are obtained in the analyses of the data for the other models. For example, in the case of the $\pm J$ Ising model at $p=0.7$, a fit of the data at $R_{\xi}=0.63$ to Eq. (64) with $\varepsilon=1.0$ gives $\eta=-0.381(5)$ for $L \geq L_{\min }=10$ $\left(\chi^{2} / \mathrm{DOF} \approx 1.6\right)$. In the case of the BDBIM at $p_{b}=0.45$, a fit of the data at $R_{\xi}=0.63$ to Eq. (64) with $\varepsilon=2.0$ gives $\eta=-0.385(4)$ for $L \geq L_{\min }=9\left(\chi^{2} / \mathrm{DOF} \approx 1.4\right)$. Taking also these results into account, we arrive at the final estimate

$$
\eta=-0.375(10)
$$

We finally discuss the behavior of the derivative $\chi^{\prime} \equiv d \chi / d \beta$ of the susceptibility, which in some numerical works, see, e.g. Ref. 30, has led to contradictory results for the exponent $\nu$. We show in the following that this discrepancy is essentially due to the analytic $O\left(L^{-1 / \nu}\right)$ corrections discussed in Sec. III, cf. Eq. (36). At fixed $R_{\xi}, \bar{\chi}^{\prime}$ is expected to behave as

$$
\bar{\chi}^{\prime}\left(L, R_{\xi}\right)=b L^{\sigma}\left[1+b_{a} L^{-1 / \nu}+\cdots+b_{\omega} L^{-\omega}+\ldots+b_{b} L^{-\sigma}+\cdots\right],
$$

where $\sigma \equiv 1 / \nu+2-\eta$. Using our best estimates of $\nu$ and $\eta$, we obtain $\sigma=2.78(4)$. Unlike the case of $\chi$ and the derivative of the phenomenological quantities, the amplitude $b_{a}$ of the $O\left(L^{-1 / \nu}\right)$ corrections does not vanish at $T_{c}$ and thus at $R_{\xi}=R_{\xi}^{*}$; therefore, it is not expected to be small when $R_{\xi} \approx R_{\xi}^{*}$. The $O\left(L^{-\sigma}\right)$ term in Eq. (66) is the background contribution, cf. Eq. (32). In Fig. 16 we show the estimates of $\sigma$ obtained in fits of $\ln \chi^{\prime}$ to

$$
\begin{aligned}
& \ln \chi^{\prime}=c+\sigma \ln L+c_{\varepsilon} L^{-\varepsilon}, \\
& \ln \chi^{\prime}=c+\sigma \ln L+c_{1} L^{-\varepsilon_{1}}+c_{2} L^{-\varepsilon_{2}}
\end{aligned}
$$

for several values of $\varepsilon, \varepsilon_{1}, \varepsilon_{2}$. The results are consistent with the expected value $\sigma \approx 2.78$, when the $O\left(L^{-1 / \nu}\right)$ corrections are taken into account.

\section{HIGH-TEMPERATURE ESTIMATES}

In the parallel-tempering simulations we have collected a large amount of data for several values of $\beta$ in the high-temperature phase. They have not been used in the analyses we have presented in the previous Sections. Here, we shall present analyses that consider all high-temperature results. They fully confirm the critical-point estimates discussed above and provide additional evidence that all models belong to the same universality class. 


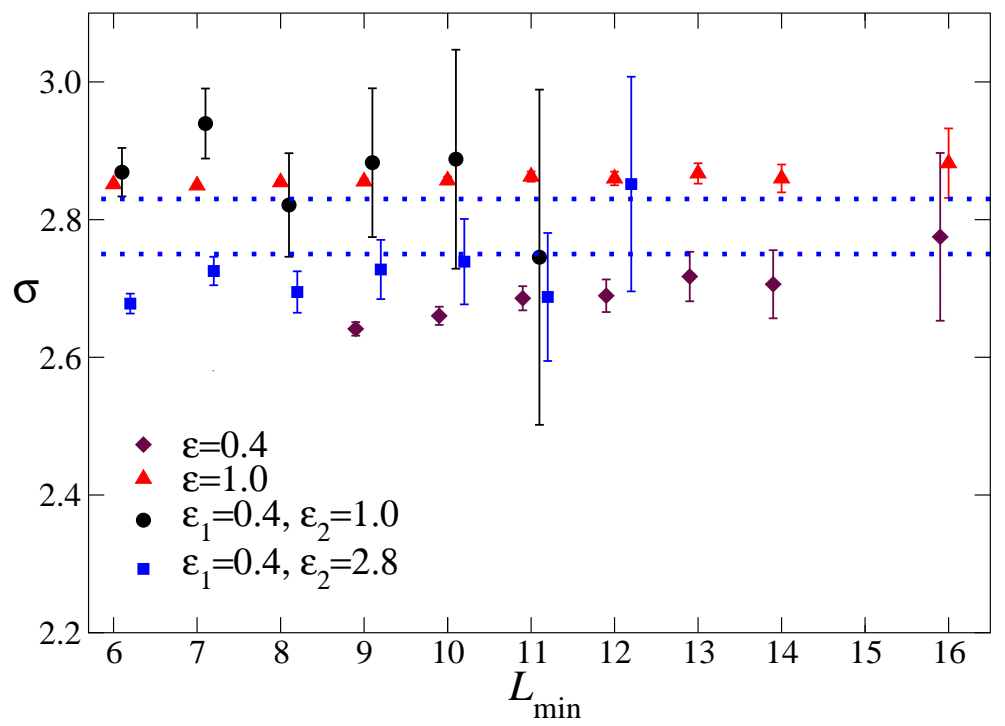

FIG. 16: (Color online) Estimates of the critical exponent $\sigma \equiv 1 / \nu+2-\eta$, as obtained by analyzing $\chi^{\prime}$ at $R_{\xi}=0.654$. The dotted lines correspond to $\sigma=2.78(4)$.

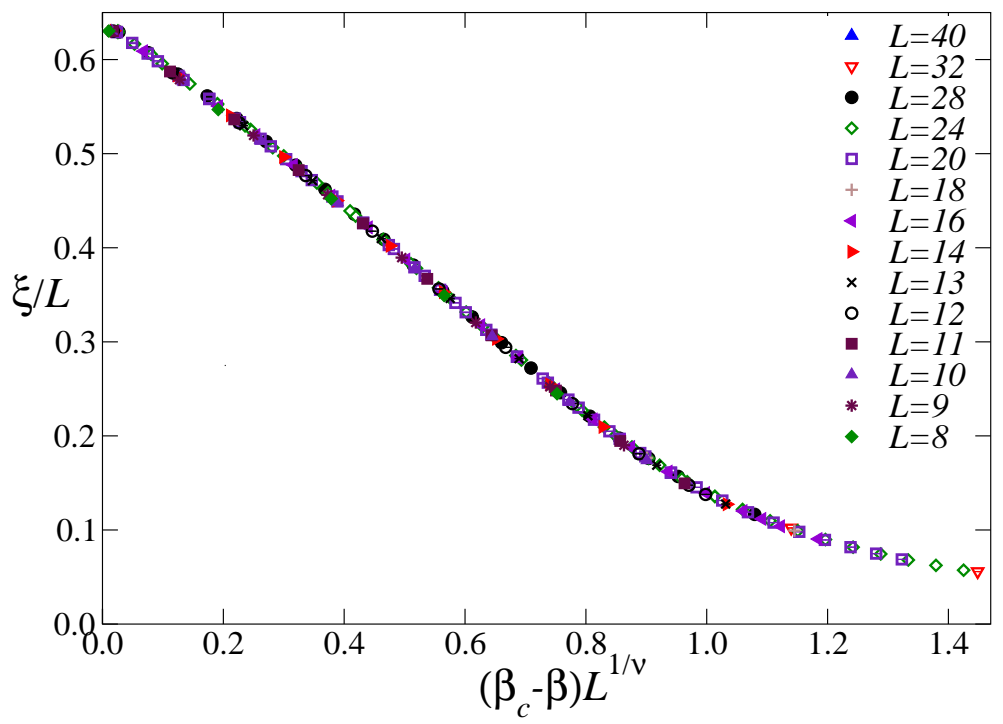

FIG. 17: (Color online) Plot of $\xi / L$ vs $\left(\beta_{c}-\beta\right) L^{1 / \nu}$ with $\beta_{c}=0.902$ and $\nu=2.45$. Data for the $\pm J$ model with $p=0.5$.

\section{A. Finite-size scaling behavior of $\xi$ and estimates of $\nu$}

We determine $\nu$ from the FSS behavior of the correlation length. The starting point is the FSS equation

$$
\frac{\xi(\beta, L)}{L}=f\left(u_{t} L^{1 / \nu}\right)+\frac{v_{\omega}(\beta)}{L^{\omega}} f_{\omega}\left(u_{t} L^{1 / \nu}\right)+\cdots
$$

where $f(x)$ and $f_{\omega}(x)$ are universal (once the normalization of the scaling fields has been fixed) and have a regular expansion in powers of $x$. In order to ensure a finite infinite-volume 
limit, for $x \rightarrow \infty$ they behave as

$$
f(x) \sim x^{-\nu}, \quad f_{\omega}(x) \sim x^{\nu(\omega-1)} .
$$

The scaling field $u_{t}$ is an analytic function of $\beta$ that vanishes at the critical point. Hence, it has an expansion

$$
u_{t}=\beta_{c}-\beta+b\left(\beta_{c}-\beta\right)^{2}+O\left[\left(\beta_{c}-\beta\right)^{3}\right] .
$$

In Fig. 17 we report $\xi(\beta, L) / L$ versus $\left(\beta_{c}-\beta\right) L^{1 / \nu}$ using the data for the $\pm J$ model at $p=0.5$ and the estimates of $\beta_{c}$ and $\nu$ obtained in the previous Sections: $\beta_{c}=0.902$ and $\nu=2.45$. The data collapse quite precisely onto a single curve. This indicates that the nonanalytic scaling corrections are small and so are the analytic ones due to the nontrivial dependence of $u_{t}$ on $\beta$.

In order to obtain quantitative estimates of $\nu$, we follow Ref. 71 and perform three different fits of $\xi(\beta, L) / L$. In the first fit we neglect the nonanalytic scaling corrections, set $u_{t}=\beta_{c}-\beta$, and fit the data to (fit a)

$$
\frac{\xi(\beta, L)}{L}=P_{n}(x)^{-\nu / n}, \quad x \equiv\left(\beta_{c}-\beta\right) L^{1 / \nu},
$$

where $P_{n}(x)$ is a polynomial of degree $n$. We have chosen polynomials for computational convenience, but any other complete set of functions can be used. Note that the specific choice (71) of fitting function satisfies the large- $x$ behavior (69). In this fit, the free parameters are the $(n+1)$ coefficients of the polynomial $P_{n}(x)$, the critical inverse temperature $\beta_{c}$, and $\nu .{ }^{72}$ The critical-point value $R_{\xi}^{*}$ is equal to $P_{n}(x=0)^{-\nu / n}$.

In order to take into account the analytic corrections (fit b), we slightly modify the Ansatz (71), setting $x \equiv \beta_{c}-\beta+b\left(\beta_{c}-\beta\right)^{2}$ and taking $b$ as an additional free parameter. Finally, we consider the nonanalytic scaling corrections. We use the Ansatz (fit $\mathrm{c}$ )

$$
\frac{\xi(\beta, L)}{L}=\left[P_{n}(x)+\frac{1}{L^{\omega}}(1+a x)^{\omega \nu} Q_{n}(x)\right]^{-\nu / n}, \quad x \equiv\left(\beta_{c}-\beta\right) L^{1 / \nu},
$$

where $P_{n}(x)$ and $Q_{n}(x)$ are both polynomials of degree $n$, and $a$ is a free parameter. We can check that Ansatz (72) has the correct infinite-volume limit. For $L \rightarrow \infty$ at fixed $\beta$, i.e., for $x \rightarrow \infty$, we have $P_{n}(x) \approx p_{n} x^{n}, Q_{n}(x) \approx q_{n} x^{n}$, and

$$
\begin{aligned}
\xi(\beta, L) & \approx L\left[p_{n} x^{n}+\frac{1}{L^{\omega}}(a x)^{\omega \nu} q_{n} x^{n}\right]^{-\nu / n} \approx L x^{-\nu}\left[p_{n}+a^{\omega \nu} q_{n}\left(\beta_{c}-\beta\right)^{\omega \nu}\right]^{-\nu / n} \\
& \sim\left(\beta_{c}-\beta\right)^{-\nu}\left[1+k\left(\beta_{c}-\beta\right)^{\Delta}\right]
\end{aligned}
$$

where $\Delta \equiv \omega \nu$. In these fits $n$ must be chosen so that $P_{n}$ provides an accurate parametrization of the scaling function. We have tried several values of $n$, until the $\chi^{2}$ of the fit does not change significantly as $n$ increases by 1 . In practice, we have taken $n$ between 6 and 10 . In fit (72) corrections to scaling are parametrized by a polynomial that has the same degree as that parametrizing the leading behavior. Our data are not so precise and corrections are not so large to require such a large number of parameters. To reduce the number of free parameters we have taken $n=6,9$ and set

$$
Q_{n}(x)=q_{0}+q_{3} x^{3}+\cdots+q_{n} x^{n} .
$$




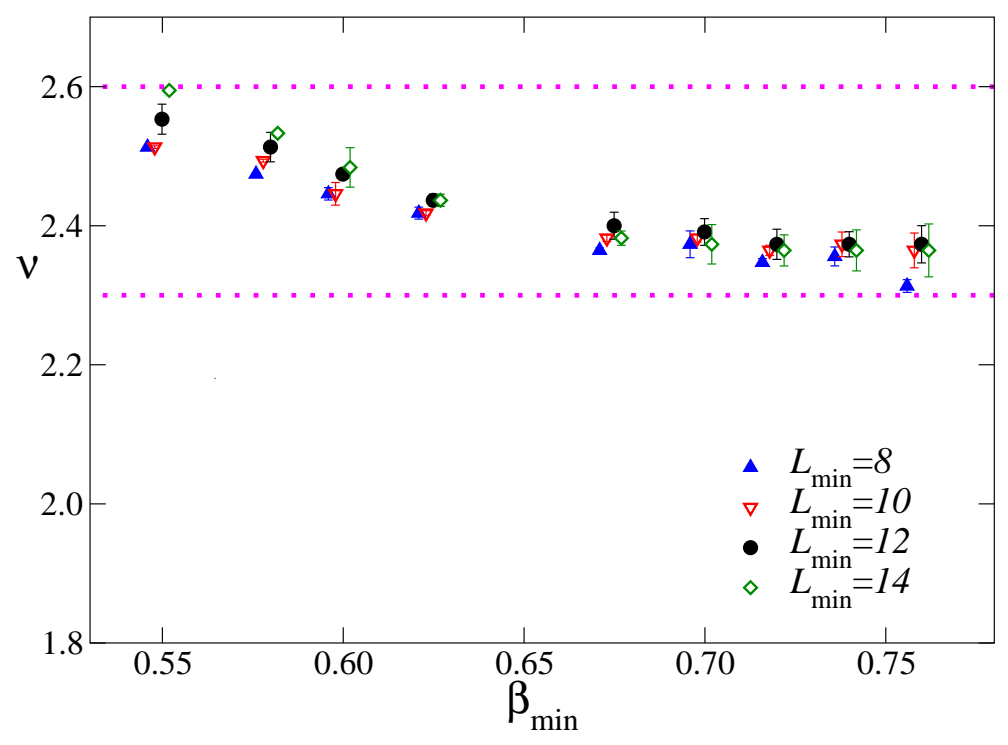

FIG. 18: (Color online) Estimates of $\nu$ for the $\pm J$ model at $p=0.5$. We report estimates obtained by fitting $\xi / L$ to the Ansatz (71) for several values of $L_{\min }$ and $\beta_{\min }$. The dotted lines correspond to the estimate $\nu=2.45(15)$.

This choice, which is completely arbitrary, significantly reduces the number of free parameters, but still allows us to parametrize accurately (at the level of the statistical errors) the scaling corrections.

In order to take into account the additional scaling corrections which are not taken into account by our fit Ansätze, we have repeated each fit several times; each time we only include data such that $\beta \geq \beta_{\min }$ and $L \geq L_{\min }$. In Fig. 18 we report the estimates of $\nu$ obtained in fits of $\xi / L$ to Eq. (71) for the $\pm J$ model at $p=0.5$. Corrections to scaling are clearly visible for small values of $\beta_{\min }$ and $L_{\text {min }}$. Indeed, at fixed $L_{\text {min }}$ the estimates decrease, becoming approximately independent of $\beta_{\min }$ for $\beta_{\min } \gtrsim 0.65$. The dependence on $L_{\min }$ is instead tiny, and all results with $L_{\min } \geq 10$ are consistent within errors.

The results of fits that also take into account the analytic and the nonanalytic corrections (with $\omega=1$ ) are reported in Fig. 19. As far as $\nu$ is concerned, no significant differences are observed and in all cases the results become independent of $\beta_{\min }$ for $\beta_{\min } \gtrsim 0.65$. All results (with their statistical errors) lie in the interval $2.3 \leq \nu \leq 2.5$, and are therefore in perfect agreement with the estimate $\nu=2.45(15)$ obtained before. Corrections to scaling are more evident in the analyses of $\beta_{c}$. Indeed, while analyses without nonanalytic scaling corrections give estimates of $\beta_{c}$ that cluster around 0.895 , those that take the corrections into account give values which are somewhat larger. In any case, all results are consistent with the estimate $\beta_{c}=0.902(8)$ obtained in Sec. VI A. Finally, these analyses also provide estimates of $R_{\xi}^{*}$. Analyses without nonanalytic scaling corrections give $R_{\xi}^{*}=0.632(5)$, while those which include scaling corrections give a somewhat higher value $R_{\xi}^{*}=0.648(5)$. Also in this case, scaling corrections appear to be quite relevant. These results are perfectly consistent with the estimate $(58), R_{\xi}^{*}=0.645(15)$. The analyses that take into account the analytic scaling corrections (fit b) also give estimates of the constant $b$ that appears in the expansion (70) of $u_{t}$ : they vary somewhat with $\beta_{\min }$ and give approximately $0 \lesssim b \lesssim 0.3$.

In order to verify universality, we compute $\nu$ and determine the FSS curves also for the 

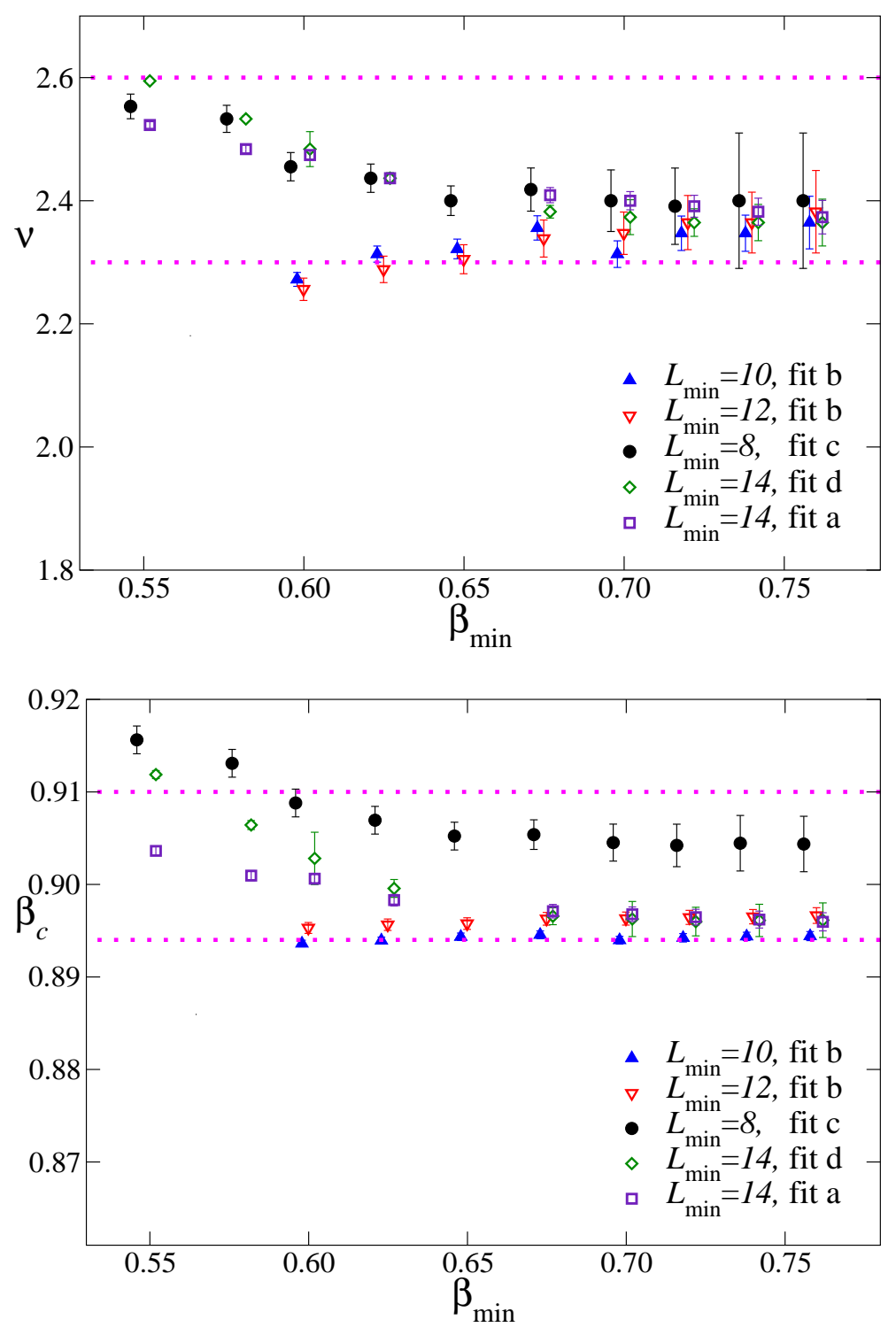

FIG. 19: (Color online) Estimates of $\nu$ (top) and $\beta_{c}$ (bottom) for the $\pm J$ model at $p=0.5$. We report estimates obtained by fitting $\xi / L$ to Eq. (71) (fit a), to Eq. (71) with $x \equiv\left[\left(\beta_{c}-\beta\right)+b\left(\beta_{c}-\right.\right.$ $\left.\beta)^{2}\right] L^{1 / \nu}$ (fit b), to Eq. (72) with $\omega=1$ (fit c), and to the extended-scaling Ansatz (86) (fit d). The dotted lines correspond to the estimates $\nu=2.45(15)$ (top) and $\beta_{c}=0.902(8)$ (bottom).

$\pm J$ model at $p=0.7$ and the BDBIM at $p_{b}=0.45$. We report here only estimates of $\nu$ obtained by fitting the data to Eq. (71), since we do not have data precise enough to allow for a detailed study of the scaling corrections. In any case, the results for $p=0.5$ indicate that scaling corrections do not play much role in the determinations of $\nu$. For the $\pm J$ model at $p=0.7$ we obtain: $\nu=2.382(5)[\nu=2.427(10)]$ for $\beta_{\min }=0.59$ and $L_{\min }=7\left[L_{\min }=9\right]$; $\nu=2.347(10)[\nu=2.382(20)]$ for $\beta_{\min }=0.68$ and the same values of $L_{\min }$. For the BDBIM at $p_{b}=0.45$ we obtain: $\nu=2.574(6)[\nu=2.637(7)]$ for $\beta_{\min }=0.82$ and again $L_{\min }=7$ $\left[L_{\min }=9\right] ; \nu=2.409(12)[\nu=2.437(18)]$ for $\beta_{\min }=1.02$. These results are in very good agreement with the estimate $\nu=2.45(15)$ reported above. 

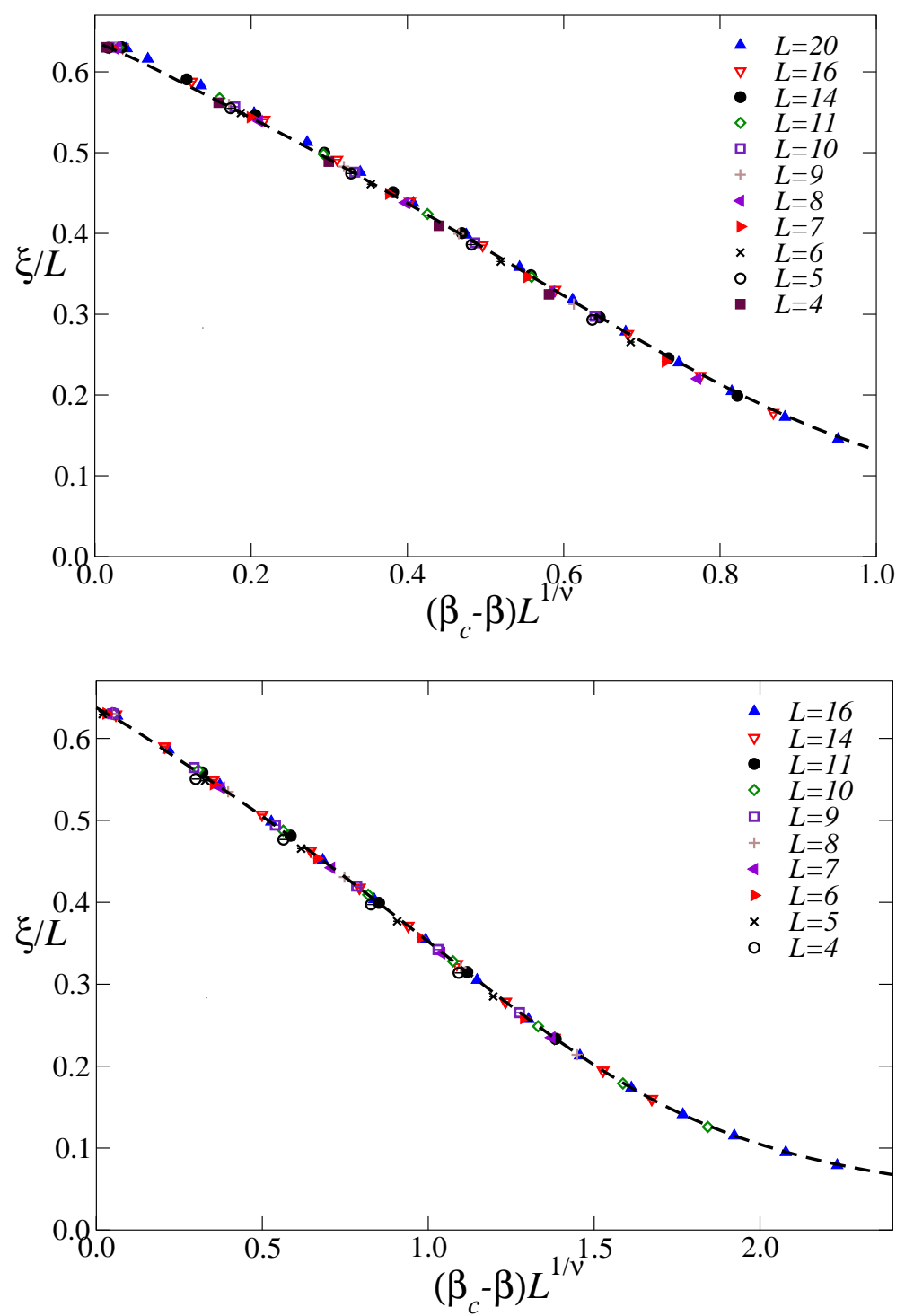

FIG. 20: (Color online) Plot of $\xi / L$ vs $\left(\beta_{c}-\beta\right) L^{1 / \nu}$ for the $\pm J$ model at $p=0.7$ (top) and for the $\mathrm{BDBIM}$ at $p_{b}=0.45$ (bottom). We use $\nu=2.45, \beta_{c}=0.87$ (top), and $\beta_{c}=1.54$ (bottom). The dashed line is the curve $R_{\xi}(c x)$, where $R_{\xi}(x)$ is the function (76), which is estimated in fits of $\xi / L$ for the $\pm J$ model at $p=0.5$, and $c$ is a model-dependent constant: $c \approx 1.026$ for the $\pm J$ model at $p=0.7$ and $c \approx 0.5641$ for the BDBIM at $p_{b}=0.45$.

In order to verify the universality of the FSS curves we first fitted the data for the $\pm J$ model at $p=0.5$ presented in Fig. 17. Taking $\nu=2.45, \beta_{c}=0.902$, and using only the data satisfying $L \geq 10, \beta \geq 0.62$, we obtain

$$
\frac{\xi}{L}=R_{\xi}(x) \quad x \equiv\left(\beta_{c}-\beta\right) L^{1 / \nu},
$$

with (this expression is valid in the interval of values of $x$ for which we have data, i.e., for $0 \leq x \lesssim 1.5)$

$$
R_{\xi}(x)=\left(6.2828+16.8612 x-39.3317 x^{2}+1926.5102 x^{3}\right.
$$




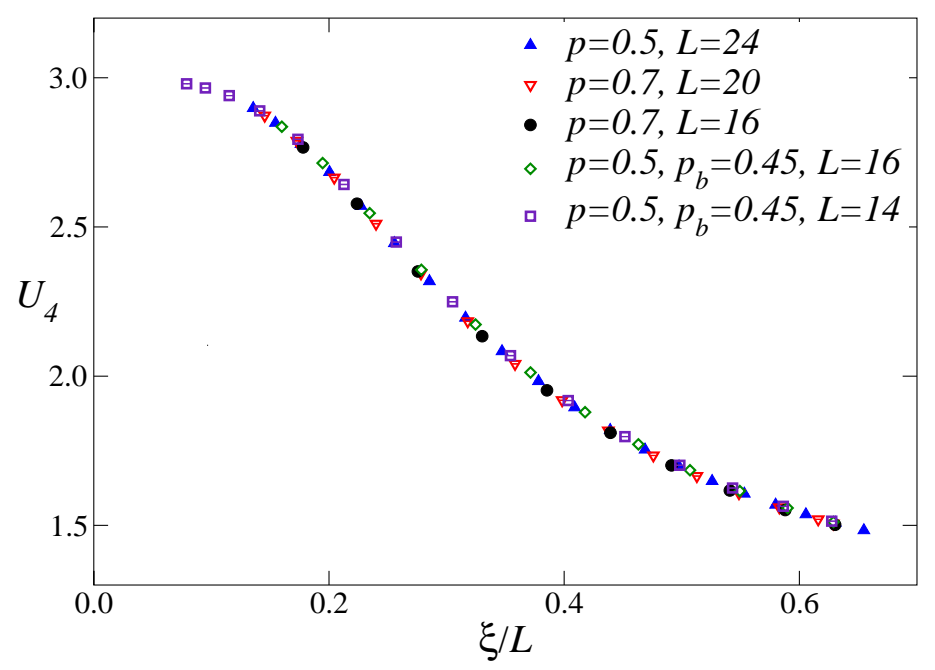

FIG. 21: (Color online) Plot of $U_{4}$ vs $\xi / L$ for the $\pm J$ model at $p=0.5$ and 0.7 and the BDBIM at $p_{b}=0.45$.

$$
\begin{aligned}
& -17659.3388 x^{4}+88711.1141 x^{5}-256918.2481 x^{6}+446776.5137 x^{7} \\
& \left.-452723.8074 x^{8}+243001.4960 x^{9}-50040.5243 x^{10}\right)^{-0.245} .
\end{aligned}
$$

The function $R_{\xi}(x)$ is universal apart from a rescaling of its argument. Thus, if we plot $\xi / L$ vs $x \equiv\left(\beta_{c}-\beta\right) L^{1 / \nu}$ in any model, the data should fall on the curve $R_{\xi}(c x)$, where $c$ is a model-dependent constant. In Fig. 20 we report the data for the $\pm J$ model at $p=0.7$ and the BDBIM at $p_{b}=0.45$. The results show very good scaling and fall on top of the curve computed from the data of the bimodal model at $p=0.5$, confirming universality.

We finally consider the cumulant $U_{4}$. In the critical limit $U_{4}$ should be a universal function of $\xi / L$, independent of the model. Corrections scale as $L^{-\omega} h(\xi / L)$, where $h(x)$ is also universal, apart from a multiplicative constant. The numerical estimates of $U_{4}$ and $\xi / L$ are reported in Fig. 21. We consider the $\pm J$ model at $p=0.5$ and $p=0.7$ and the BDBIM at $p_{d}=0.45$. We only consider the largest lattices, so that nonanalytic scaling corrections are not visible on the scale of the figure (a detailed study of the $L^{-\omega}$ corrections for $\xi / L=0.63$ is reported in Sec. VA). All points fall quite precisely onto a single curve, confirming that the PG transition in these three models belongs to the same universality class.

\section{B. Finite-size scaling of the susceptibility and estimates of $\eta$}

We now turn to the determination of the critical exponent $\eta$. The starting point is Eq. (29), which we rewrite as

$$
\chi(\beta, L)=L^{2-\eta} \bar{u}_{h}(\beta)^{2} g\left(u_{t} L^{1 / \nu}\right)\left[1+\frac{v_{\omega}(\beta)}{L^{\omega}} g_{\omega}\left(u_{t} L^{1 / \nu}\right)+\ldots\right]
$$

This equation is not very convenient since it involves $u_{t}$, hence the critical temperature, and the exponent $\nu$. To reduce the number of unknown parameters, we note that Eq. (68) can 

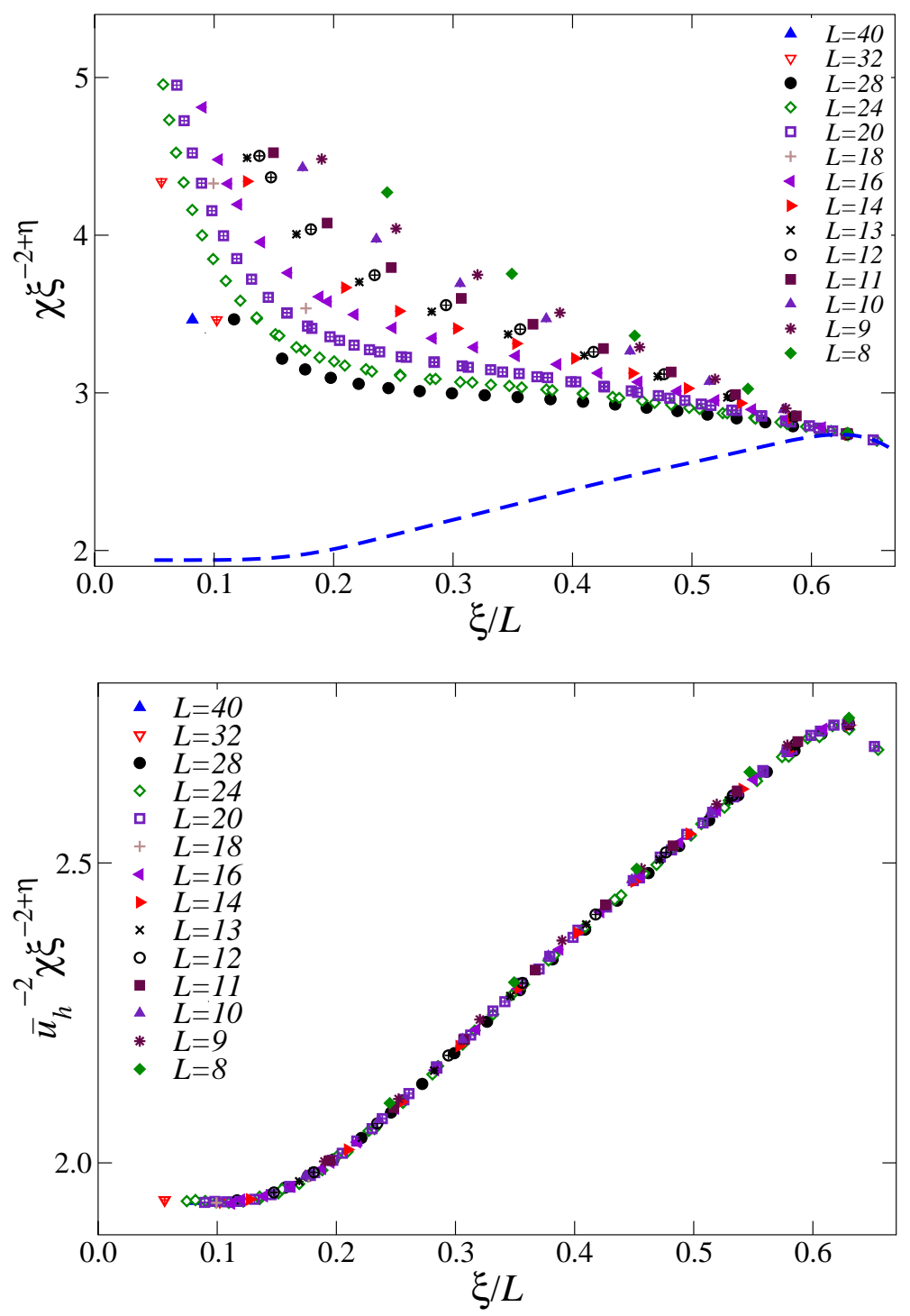

FIG. 22: (Color online) Plot of $\chi \xi^{\eta-2}$ (upper panel) and of $\chi \xi^{\eta-2} \tilde{u}_{h}^{-2}$ (lower panel) vs $\xi / L$ with $\eta=-0.375$. The dashed line in the upper panel is the universal curve $\tilde{C}(\xi / L)$, as estimated in fits of $\chi$ to Eq. (80). Data for the $\pm J$ model at $p=0.5$.

be inverted to give

$$
u_{t} L^{1 / \nu}=F(\xi / L)+\frac{v_{\omega}(\beta)}{L^{\omega}} F_{\omega}(\xi / L)+\ldots
$$

Inserting in Eq. (77) we obtain the scaling form

$$
\chi(\beta, L)=\xi^{2-\eta} \bar{u}_{h}(\beta)^{2} C(\xi / L)\left[1+v_{\omega}(\beta) \xi^{-\omega} C_{\omega}(\xi / L)+\ldots\right] .
$$

In Eq. (79) we have singled out $\xi^{2-\eta}$ and $\xi^{-\omega}$ instead of $L^{2-\eta}$ and $L^{-\omega}$. With this choice $C(x)$ and $C_{\omega}(x)$ are regular for $x \rightarrow 0$. Note the presence of the function $\bar{u}_{h}(\beta)$. In the FSS limit $\xi \rightarrow \infty, L \rightarrow \infty$, at fixed $\xi / L$, we always have $\beta \rightarrow \beta_{c}$, so that asymptotically it should be possible to replace $\bar{u}_{h}(\beta)$ with the constant $\bar{u}_{h}\left(\beta_{c}\right)$. Therefore, this function gives rise to scaling corrections, that we have named analytic corrections in the previous sections. 


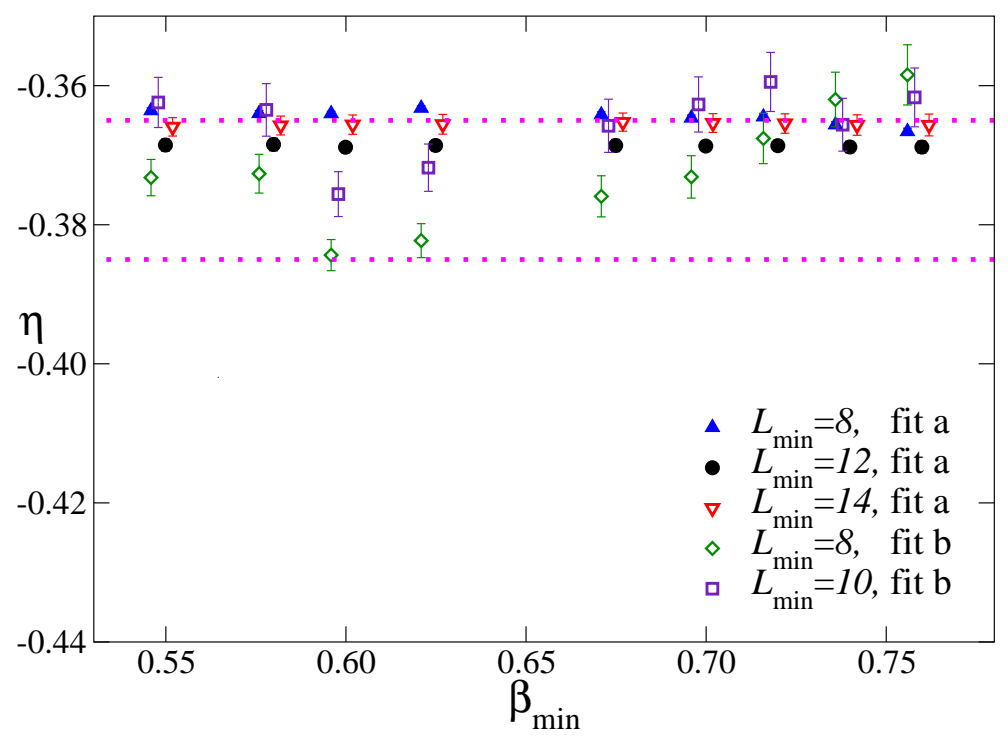

FIG. 23: (Color online) Estimates of $\eta$ for the $\pm J$ model at $p=0.5$. We report estimates obtained by fitting $\chi$ to the Ansatz (80) (fit a) and (81) (fit b) for several values of $L_{\min }$ and $\beta_{\min }$. The dotted lines correspond to the estimate $\eta=-0.375(10)$.

In order to understand their relevance for our data, in Fig. 22 (upper panel) we plot $\xi^{\eta-2} \chi$ versus $\xi / L$ for the $\pm J$ model at $p=0.5$. It is evident that the data do not fall onto a single curve: the scaling-field term $\bar{u}_{h}(\beta)$ varies significantly with $\beta$ and therefore cannot be neglected.

The previous discussion indicates that, in order to estimate accurately the exponent $\eta$, it is essential to include the analytic corrections in the fitting function. We perform two fits. In the first one (fit a) we neglect the nonanalytic scaling corrections and consider

$$
\ln \frac{\chi}{\xi^{2}}=-\eta \ln \xi+P_{n}(\xi / L)+Q_{m}(\beta)
$$

where $P_{n}(x)$ and $Q_{m}(x)$ are polynomials of degree $n$ and $m$, respectively. Moreover, we require $Q_{m}(0)=0$ in order to avoid the presence of two constant terms. As before, $n$ and $m$ are varied till the quality of the fit does not change significantly by varying the parameters by 1 . In practice, we take $6 \leq n, m \leq 10$. To include the scaling corrections, we also consider

$$
\ln \frac{\chi}{\xi^{2}}=-\eta \ln \xi+P_{n}(\xi / L)+Q_{m}(\beta)+\xi^{-\omega} S_{p}(\xi / L)
$$

where $S_{p}(x)$ is a polynomial of degree $p$ : we take $p \leq 3$. Note that here, as we already did in the analysis of $\xi / L$, we neglect the $\beta$ dependence of the scaling field $v_{\omega}$.

The results of the fits for the $\pm J$ model at $p=0.5$ are reported in Fig. 23. The estimates of the fit to Eq. (80) are very stable and show a very tiny dependence on $\beta_{\min }$ and $L_{\min }$. For instance, for $\beta_{\min }=0.55$ we obtain $\eta=-0.3636(8)\left(L_{\min }=8\right), \eta=-0.3659(27)$ $\left(L_{\min }=14\right)$, while for $\beta_{\min }=0.75$ we have $\eta=-0.3666(10)\left(L_{\min }=8\right), \eta=-0.3657(31)$ $\left(L_{\min }=14\right)$. Fits with nonanalytic scaling corrections are less stable. We observe significant fluctuations that indicate that the data are not precise enough to be sensitive to this type of 


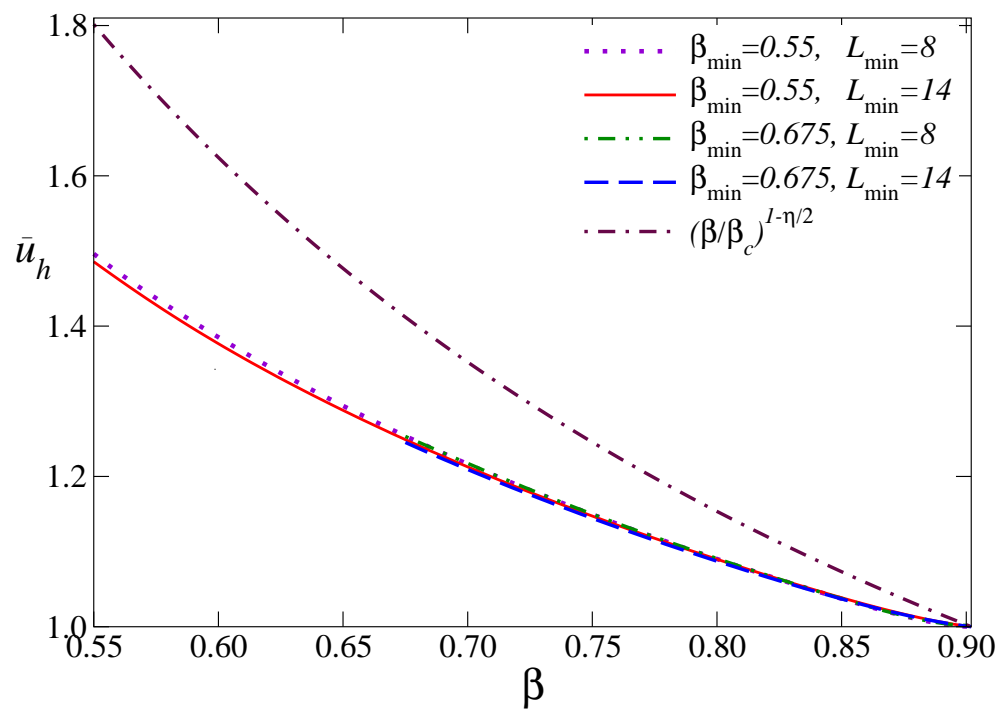

FIG. 24: (Color online) Scaling-field function $\tilde{u}_{h}(\beta)$ for the $\pm J$ model at $p=0.5$, as determined in the fits of $\chi$ to the Ansatz (80), for different values of $\beta_{\min }$ and $L_{\min }$. It is normalized such that $\tilde{u}_{h}\left(\beta_{c}\right)=1$. We also report the approximation $\tilde{u}_{h}(\beta) \approx\left(\beta / \beta_{c}\right)^{1-\eta / 2}$, which is used in the extended-scaling scheme (see the discussion in Sec. VIIC).

scaling corrections. This is consistent with what is observed at the critical point: While the results for $\eta$ depend strongly on the chosen value for $R_{\xi, f}$, indicating that the analytic scaling corrections are important, essentially no dependence is observed on the nonanalytic ones; see, e.g., Fig. 15. In any case, all results are consistent with the estimate $\eta=-0.375(10)$ obtained in Sec. VIC.

The fits also give estimates of the function $\bar{u}_{h}(\beta)$ that appears in Eqs. (77) and (79). In Fig. 24 we plot $\tilde{u}_{h}(\beta)=\bar{u}_{h}(\beta) / \bar{u}_{h}\left(\beta_{c}\right)\left[\tilde{u}_{h}(\beta)\right.$ is normalized so that $\left.\tilde{u}_{h}\left(\beta_{c}\right)=1\right]$ as obtained in the different fits. The results corresponding to different values of $L_{\text {min }}$ and $\beta_{\min }$ agree nicely, supporting the scaling Ansatz (79). A simple expression which reproduces the results reported in Fig. 24 is

$$
\tilde{u}_{h}(\beta)=1+0.556247(1-\beta / 0.902)+1.83322(1-\beta / 0.902)^{2},
$$

which is valid for $0.55 \leq \beta \leq 0.902$. Once $\tilde{u}_{h}(\beta)$ has been determined, we can compute the scaling function $\tilde{C}(\xi / L)=\bar{u}_{h}\left(\beta_{c}\right)^{2} C(\xi / L)$ by considering $L^{\eta-2} \chi \tilde{u}_{h}(\beta)^{-2}$. Such a combination is shown in Fig. 22 (lower panel): all points fall on top of each other, confirming the validity of the FSS Ansatz. Moreover, as expected, we find that $\tilde{C}(0)$ is finite and $\tilde{C}(\xi / L)$ is approximately constant for $\xi / L \lesssim 0.15$, two properties which are not obvious from the upper panel of Fig. 22. These conclusions are consistent with the FSS results for $\chi(2 L, \beta) / \chi(L, \beta)$ (in this quantity the analytic function $\bar{u}_{h}(\beta)$ cancels out) reported in Refs. 20,28, which show that this ratio has a tiny dependence on $\xi / L$ up to $\xi / L \approx 0.15$ (for $\xi / L=0.15$ we have $\chi(2 L, \beta) / \chi(L, \beta) \approx 1.02$ ). Note that the curve in the lower panel of Fig. 22 (which corresponds to the dashed line in the upper panel) is the limiting curve of the points that appear in the upper panel as $L \rightarrow \infty$. Since the rate of convergence is very slow (at fixed $\xi / L$ data converge as $L^{-1 / \nu}$ ), it is clear that such an asymptotic behavior can only be observed on enormously large lattices. Thus, in order to estimate $\eta$, it is crucial to take the function 
$\bar{u}_{h}(\beta)$ into account.

The function $\tilde{C}(x)$ is universal, apart from a model-dependent multiplicative constant. We write it as

$$
\tilde{C}(x)=b \Gamma(x) \quad \Gamma(0)=1,
$$

where $\Gamma(x)$ is universal. A fit of the data reported in Fig. 22 gives

$$
\begin{aligned}
& \Gamma(x)=1+5.9622 y-104.4625 y^{2}+1516.2443 y^{3} \\
& \quad-12260.6638 y^{4}+50105.6104 y^{5}-80471.2150 y^{6}, \\
& y \equiv \exp (-1 / x)
\end{aligned}
$$

and $b \approx 1.9395$. The expression (84) is valid for $x \equiv \xi / L \lesssim R_{\xi}^{*} \approx 0.645$.

The same analyses can be repeated for the $\pm J$ model at $p=0.7$ and the BDBIM at $p_{b}=0.45$. Here we only present results corresponding to fits to the Ansatz (80). Our data are not precise enough to allow us to perform fits which include the nonanalytic scaling corrections. The results are consistent with the estimate $\eta=-0.375(10)$. For the $\pm J$ model at $p=0.7$, we obtain $\eta=-0.366(2)\left(\beta_{\min }=0.59\right)$ and $\eta=-0.366(3)\left(\beta_{\min }=0.68\right)$ for $L_{\min }=7$, and $\eta=-0.368(2)\left(\beta_{\min }=0.59\right)$ and $\eta=-0.366(3)\left(\beta_{\min }=0.68\right)$ for $L_{\min }=9$. For the BDBIM, we obtain $\eta=-0.361(2)$ and $-0.359(3)$ for $L_{\min }=7$ and 9 and any $\beta_{\min }$ in the range $[0.82,1.12]$.

As a further check of universality we determine the scaling behavior of the combination $\chi \xi^{\eta-2} \tilde{u}_{h}^{-2}$. In Fig. 25 we report this quantity for the $\pm J$ model at $p=0.7$ (upper panel) and the BDBIM at $p_{b}=0.45$ (middle panel), using in both cases $\eta=-0.375$ and our best estimate of $\tilde{u}_{h}$. As expected all points fall onto a single curve. If universality holds, these curves should be parametrized as $b \Gamma(x)$, where $\Gamma(x)$ is given in Eq. (84) and $b$ is a modeldependent constant. In the case of the $\pm J$ model (upper panel of Fig. 25) we observe a very good agreement, while for the BDBIM at $p_{b}=0.45$ (middle panel) some discrepancies occur for $\xi / L \gtrsim 0.4$. There are two reasons for them. First, the function $\tilde{u}_{h}$ is not precisely known for $\beta \approx \beta_{c}$ : in this range of values of $\beta$ it varies slightly $(10 \%)$ with $\beta_{\min }$ and $L_{\min }$. Second, the plot depends on $\eta$. If we use $\eta=-0.360$, which is the value obtained in the fits for the diluted model which provide $\tilde{u}_{h}$, we obtain the lower panel of Fig. 25. Discrepancies are now significantly reduced.

It is worth noting that the functions $\bar{u}_{h}$ are approximately the same in the three models we study, if one considers them as a function of the reduced temperature $t \equiv 1-\beta / \beta_{c}$. For $0 \lesssim t \lesssim 0.4$ - this is the interval of $t$ which is probed by our simulations - the ratio $\bar{u}_{h \text {,model1 }}(t) / \bar{u}_{h \text {,model2 }}(t)$ is constant, within our precision, for any pair of models. This result is somewhat unexpected within RG theory, because these functions are not universal.

Finally, let us comment on the FSS approach of Ref. 73, applied to spin-glass systems in Refs. 20,28. In this approach one considers the ratio $\chi(2 L, \beta) / \chi(L, \beta)$. This choice has a significant advantage: the scaling-field function $\bar{u}_{h}(\beta)$ cancels out, so that the leading scaling corrections are the nonanalytic ones. As we have shown here, they are quite small, so that very good scaling is observed and reliable infinite-volume estimates are obtained. Analytic scaling corrections come in again when considering the critical limit of the infinite-volume results $\chi_{\infty}(\beta)$. Indeed, since $\Delta \equiv \omega \nu \approx 2.45$, for $\beta \rightarrow \beta_{c}$ the analytic corrections dominate:

$$
\chi_{\infty}(\beta)=\left(\beta_{c}-\beta\right)^{-\gamma}\left[b_{0}+b_{1}\left(\beta_{c}-\beta\right)+b_{2}\left(\beta_{c}-\beta\right)^{2}+b_{\Delta}\left(\beta_{c}-\beta\right)^{\Delta}+\cdots\right] .
$$



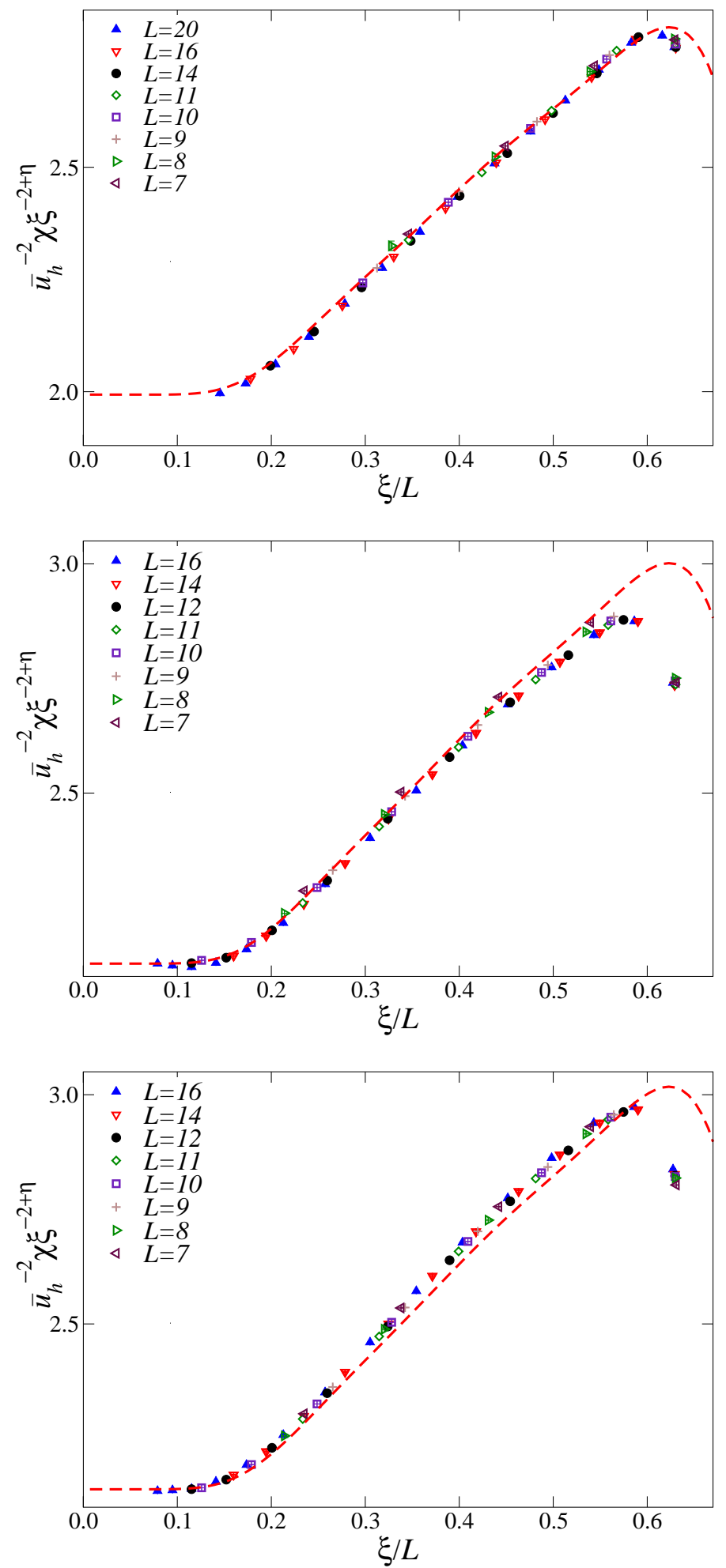

FIG. 25: (Color online) Plot of $\chi \xi^{\eta-2} \tilde{u}_{h}^{-2}$ vs $\xi / L$ for the $\pm J$ model at $p=0.7$ with $\eta=-0.375$ (upper panel) and for the BDBIM at $p_{b}=0.45$ with $\eta=-0.375$ (middle panel) and $\eta=-0.360$ (lower panel). In each panel we report the curve $b \Gamma(\xi / L)$, where $\Gamma(\xi / L)$ is defined in Eq. (84) and $b$ is a model-dependent constant. We use $b=1.99346,2.12794,2.13923$, in the upper, middle, and lower panel, respectively. They are determined by requiring a perfect fit for $\xi / L \leq 0.2$. 

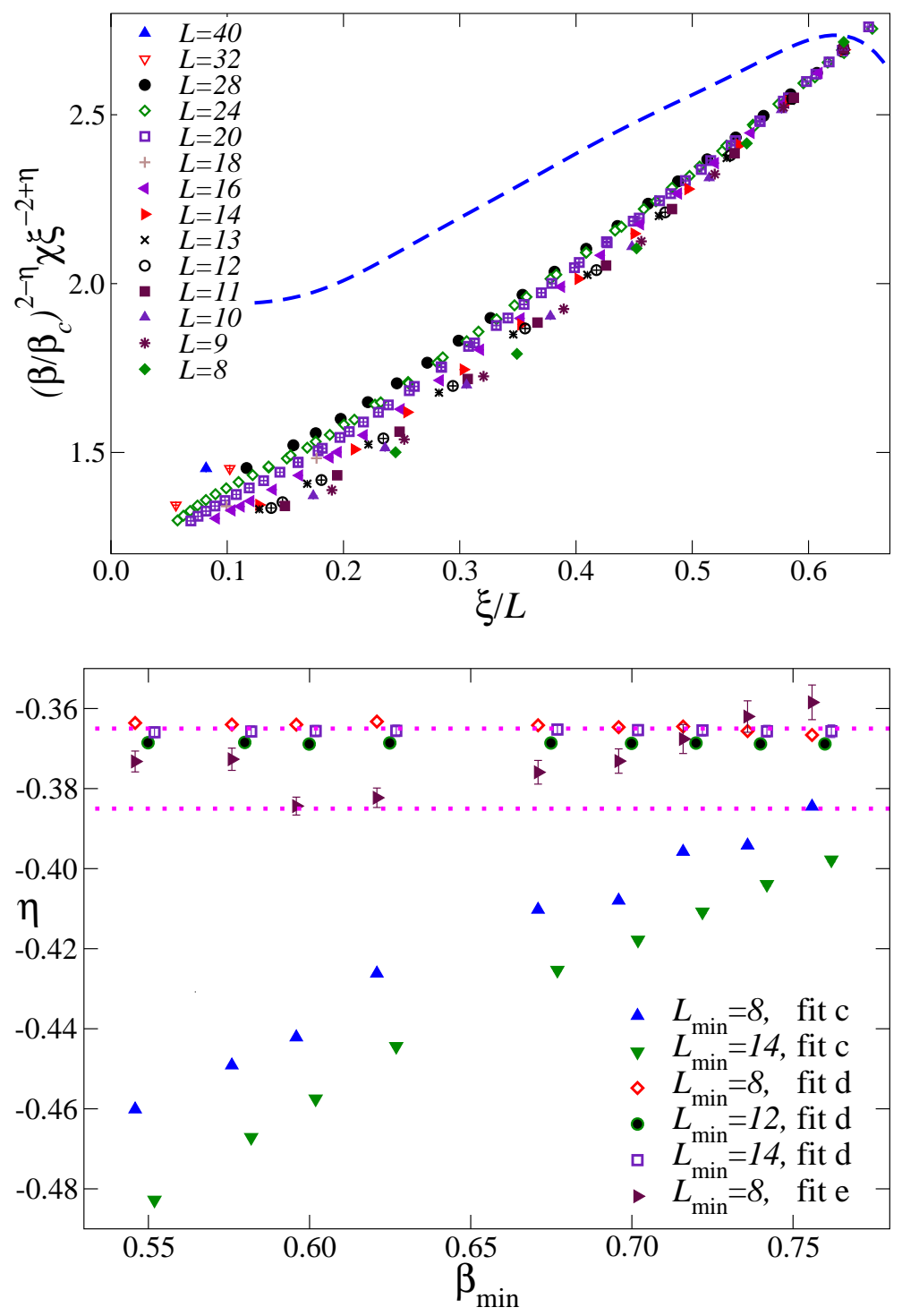

FIG. 26: (Color online) Extended-scaling results for the $\pm J$ model at $p=0.5$. In the upper panel we report $\beta^{2-\eta} \chi \xi^{\eta-2}$ vs $\xi / L$. The dashed line is the universal curve $\tilde{C}(\xi / L)$, as estimated in fits of $\chi$ to Eq. (80). In the lower panel we report estimates of $\eta$ obtained in three different fits for several values of $L_{\min }$ and $\beta_{\text {min }}$. Fit c uses the Ansatz (89), fit $\mathrm{d}$ and e are defined in text, below Eq. (89). The lines correspond to the estimate $\eta=-0.375(10)$.

\section{Extended-scaling scheme}

In this Section we consider the extended-scaling scheme introduced in Ref. 29. It consists in a particular choice of scaling variables, which, according to Ref. 29, should somehow decrease scaling corrections and thus allow a faster convergence to the critical limit. Let us consider first $\xi / L$. In this scheme the appropriate fit Ansatz is

$$
\frac{\xi(\beta, L)}{L}=P_{n}(x)^{-\nu / n}, \quad x \equiv\left(\beta_{c}^{2}-\beta^{2}\right)(L / \beta)^{1 / \nu}
$$


where $P_{n}(x)$ is a polynomial of degree $n$. The results for the $\pm J$ model at $p=0.5$ are reported in Fig. 19 and should be compared with those obtained by fits to Eq. (71), which neglect any scaling corrections (fit a). They are substantially equivalent and no improvement is observed. This can be explained by noting that, for the present values of $\beta_{c}$ and $\nu$, we have

$$
\left(\beta_{c}^{2}-\beta^{2}\right) \beta^{-1 / \nu}=1.88\left(\beta_{c}-\beta\right)\left[1-0.10\left(\beta_{c}-\beta\right)+\ldots\right] .
$$

Thus, fit (86) is essentially equivalent to a fit with analytic corrections (fit b) with $b=-0.10$. Such a value of $b$ is small-hence, this change of the scaling variable does not have much influence on the final results - and is close to what we obtain numerically, though not fully consistent (we predict $0 \lesssim b \lesssim 0.3$ ). We also tried fits with $x \equiv\left(\beta_{c}^{2}-\beta^{2}\right) L^{1 / \nu}$, which should be the natural variable in spin-glass systems, given the symmetry under $\beta \rightarrow-\beta .{ }^{24}$ These fits are significantly worse than the previous ones for $\beta \lesssim 0.70$. For larger values, no significant differences are observed. These results can be understood by noting $\left(\beta_{c}^{2}-\beta^{2}\right)=$ $1.80\left(\beta_{c}-\beta\right)\left[1-0.55\left(\beta_{c}-\beta\right)+\ldots\right]$. Thus, this choice of scaling variable corresponds to assuming $b=-0.55$ in Eq. (70), which is significantly larger than what we find numerically. Therefore, if we use $\left(\beta_{c}^{2}-\beta^{2}\right)$ as approximate thermal scaling field, the analytic correctionsin this case they are proportional to $\left(\beta_{c}^{2}-\beta^{2}\right)^{2}$ - are more important than in the case in which $u_{t}$ is simply approximated with $\beta_{c}-\beta$.

The extended-scaling scheme can also be applied to the analysis of the susceptibility. It amounts to consider the scaling Ansatz ${ }^{29}$

$$
\chi(\beta, L)=\beta^{\eta-2} \xi^{2-\eta} C(\xi / L) .
$$

In Fig. 26 we show $\beta^{2-\eta} \chi \xi^{\eta-2}$ versus $\xi / L$ for the $\pm J$ model at $p=0.5$. Scaling is better than that observed in the upper panel of Fig 22: the scatter of the data points is significantly reduced, indicating that $\beta^{\eta-2}$ approximates the scaling-field term $\bar{u}_{h}^{2}$ better than a constant. However, the rescaled data are still far from the asymptotic curve (the dashed line) determined numerically above, indicating that the residual analytic scaling corrections are also in this case not negligible. This is better understood, by comparing the function $\tilde{u}_{h}$, as determined in the fits, with the approximation $\left(\beta / \beta_{c}\right)^{\eta / 2-1}$, which follows from Eq. (88). As can be seen from Fig. 24, the approximate expression proposed in Ref. 29 has the correct qualitative shape, but differs significantly from the quantitative point of view. For these reasons, we do not expect the scaling Ansatz (88) to be particularly useful to estimate $\eta$ from our data.

To understand the role of the residual analytic scaling corrections on the determinations of $\eta$, we fit the data for the $\pm J$ model at $p=0.5$ to the scaling Ansatz (fit c)

$$
\ln \frac{\chi \beta^{2}}{\xi^{2}}=-\eta \ln \frac{\xi}{\beta}+P_{n}(\xi / L)
$$

where $P_{n}(x)$ is a polynomial of degree $n$. The results are reported in Fig. 26. They vary strongly with $\beta_{\min }$ and $L_{\min }$, indicating that scaling corrections are sizable and not negligible. As a test we have also repeated the fits to Eqs. (80) and (81) replacing $\ln \chi / \xi^{2}$ with $\ln \chi \beta^{2} / \xi^{2}$ and $\ln \xi$ with $\ln \xi / \beta$ (we call fit $d$ and fit e the fits corresponding to Eqs. (80) and (81), respectively). As expected, the results are identical to those obtained in fit b and fit $\mathrm{c}$, respectively. Indeed, the fits only differ in the parametrization of the analytic function $\bar{u}_{h}$. Note that the extended-scaling approximation for $\bar{u}_{h}$ is not analytic in $\beta$, since $\beta=$ 0 is a branching point. This is, however, irrelevant in practice, since we are looking for approximations of $\bar{u}_{h}$ in the interval $0.6 \lesssim \beta \lesssim 0.9$, which is quite far from $\beta=0$. 


\section{THE ZERO-MOMENTUM QUARTIC COUPLINGS $G_{4}$ AND $G_{22}$ IN THE HIGH-TEMPERATURE PHASE}

In this section we consider the zero-momentum quartic couplings $G_{4}$ and $G_{22}$ defined in Eq. (19) and (20) and estimate their infinite-volume critical value defined by

$$
G_{\#}^{*}=\lim _{\beta \rightarrow \beta_{c}^{+}} \lim _{L \rightarrow \infty} G_{\#}(L, \beta) .
$$

We consider the $\pm J$ Ising model at $p=0.5$ and use the estimates of the quartic couplings for $\beta=0.55$ and $L=12,14,16,18$, and 32 , and for $\beta=0.625$ and $L=16,18,28,32$, and 40 . We combine results obtained in the random-exchange runs that we performed for our FSS study around $\beta_{c}$ and results obtained in standard MC simulations for these two values of $\beta{ }^{74}$ First, we investigate the infinite-volume limit. The correlation length converges rapidly (see Fig. 27). For instance, for $\beta=0.55$ we obtain $\xi=1.7888(3), 1.7885(5), 1.7872(3)$ for $L=18,20,32$, while for $\beta=0.625$ we have $\xi=3.2694(12), 3.2709$ (13) for $L=32$ and $L=$ 40: for $L / \xi \gtrsim 10$ the results vary by less than $0.1 \%$, indicating that the difference from their thermodynamic limit is within $0.1 \%$. Thus, we can take as infinite-volume estimates those obtained on the largest lattices. The quartic couplings show larger finite-size corrections. As shown in Figs. 27 the infinite-volume limit is approximately reached for $L / \xi \gtrsim 12$, within our statistical precision. Indeed at $\beta=0.625$ we find $G_{4}=90.46(9), 90.27(16), 90.23(29)$ and $G_{22}=-16.53(5),-16.36(9),-16.40(9)$ for $L=18,20,32$, respectively. We can thus take the estimates on the largest lattices as infinite-volume estimates.

In the critical limit we expect

$$
G_{\#}(L=\infty, \beta)=G_{\#}^{*}+c_{\#} \xi^{-\omega},
$$

with $\omega=1.0(1)$. The comparison of the results at $\beta=0.55$ and $\beta=0.625$ leads us to the estimates

$$
G_{4}^{*}=90.3(5), \quad G_{22}^{*}=-17(1),
$$

where the error takes also into account the effects of the $O\left(\xi^{-\omega}\right)$ scaling corrections, which is roughly estimated from the difference of the infinite-volume results at the two values of $\beta$. These results can be compared with those obtained in Ref. 27 for the random-anisotropy Heisenberg model in the limit of infinite anisotropy: $G_{4}^{*}=88(8)$ and $G_{22}^{*}=-11(4)$. There is a substantial agreement, which confirms that the critical behavior of the random-anisotropy Heisenberg model for infinite anisotropy belongs to the Ising spin-glass universality class. ${ }^{27,35}$

\section{CONCLUSIONS}

In this paper we discuss the critical behavior of three-dimensional Ising spin-glass systems with the purpose of verifying universality, clarifying the role of scaling corrections, and determining the critical exponents. More precisely, our results can be summarized as follows.

i) By using the $\mathrm{RG}$ we derive the behavior for $L \rightarrow \infty$ and $\beta \rightarrow \beta_{c}$ of several quantities which are routinely measured in $\mathrm{MC}$ simulations. In particular, we show that the analytic dependence of the scaling fields on the model parameters may give rise to corrections which behave as $L^{-1 / \nu} \sim L^{-0.4}$. If they are neglected, FSS analyses give 

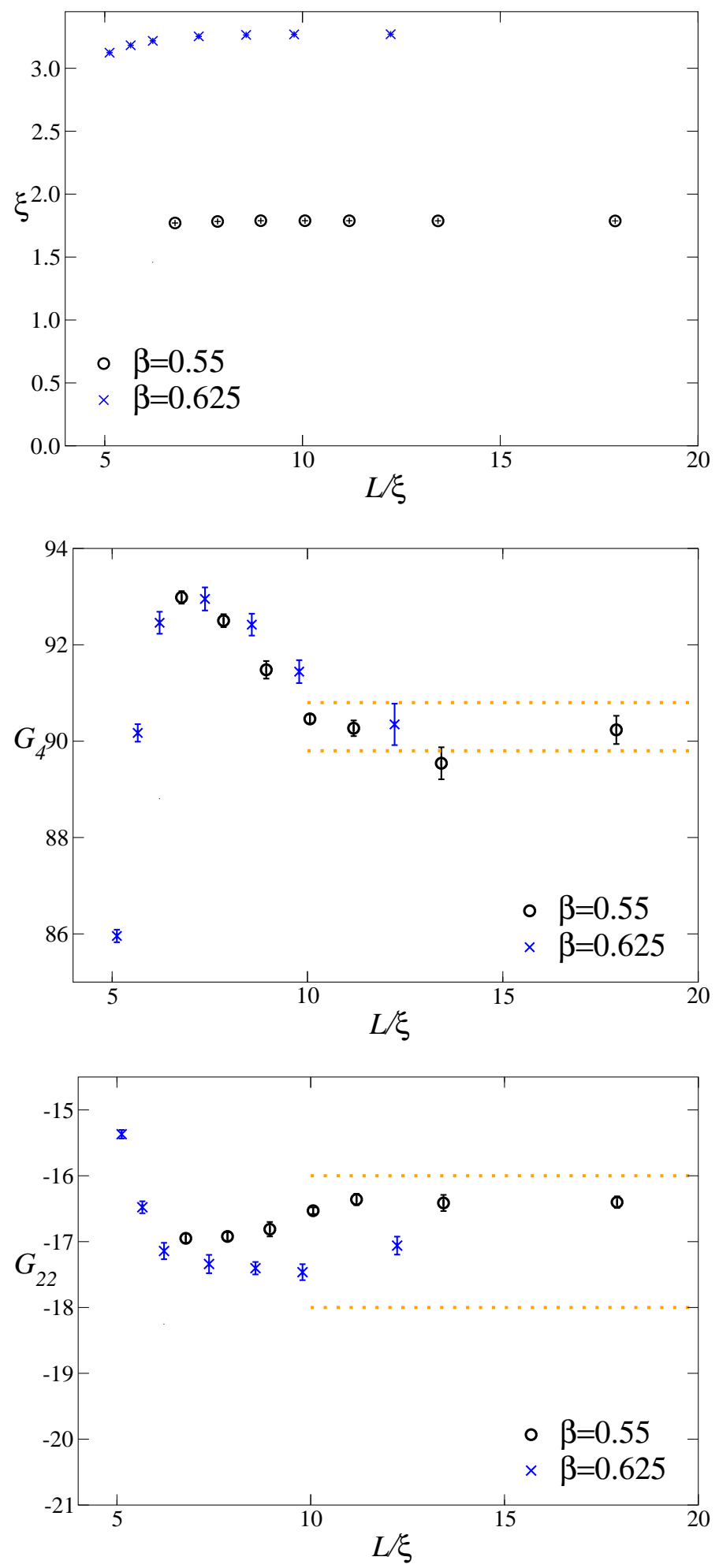

FIG. 27: (Color online) Estimates of $\xi(L, \beta)$ (top), $G_{4}(L, \beta)$ (middle), and $G_{22}(L, \beta)$ (bottom) versus $L / \xi$ for $\beta=0.55$ and $\beta=0.625$, corresponding to the infinite-volume correlation lengths $\xi_{\infty} \approx 1.79$ and $\xi_{\infty} \approx 3.27$. Results for the $\pm J$ model at $p=0.5$. The dotted lines correspond to the estimates $G_{4}^{*}=90.3(5)$ (middle panel), $G_{22}^{*}=-17(1)$ (lower panel). 
inconsistent results. These corrections have been overlooked in previous FSS studies. Note that the general expressions we obtain are relevant also in other glassy systems, in which $\nu$ is typically large.

ii) We determine the leading nonanalytic correction-to-scaling exponent $\omega$. We obtain $\omega=1.0(1)$. Note that in Ising spin-glass systems nonanalytic scaling corrections decay faster than in the Ising model, in which $\omega \approx 0.8$, see Ref. 47 . The exponent $\omega$ is also significantly larger than that at the PF transition, which occurs for small frustration: $\omega=0.29(2) \cdot{ }^{75}$

iii) We accurately verify universality. A careful analysis of the quartic cumulants $U_{4}$ and $U_{22}$ at fixed $R_{\xi}=0.63$ shows that their limit for $L \rightarrow \infty$ is independent of the model and of the disorder distribution. The results obtained in the different models differ at most by approximately one per mille in the case of $\bar{U}_{4}$ and one per cent for $\bar{U}_{22}$. They support the existence of a unique Ising spin-glass universality class. Universality is also supported by the FSS analyses of $\xi$ and $\chi$ in the high-temperature phase. We verify that the FSS curves for these two quantities are independent of the model.

iv) We determine the critical exponents. For this purpose we perform analyses at the critical point and analyses which take into account all high-temperature data. Results are consistent, once the analytic and the nonanalytic corrections are taken into account. Moreover, they do not depend on the model and disorder distribution. Again, this supports the universality of the paramagnetic-glassy transition. We obtain

$$
\nu=2.45(15), \quad \eta=-0.375(10) .
$$

Using scaling and hyperscaling relations, we find $\beta=\nu(1+\eta) / 2=0.77(5), \gamma=$ $(2-\eta) \nu=5.8(4)$, and $\alpha=2-3 \nu=-5.4(5)$.

Our estimates of the critical exponents can be compared with those reported in the literature. Earlier estimates of $\nu$ are reported in the introduction and in Table I of Ref. 30. Some of the most recent ones are close to our final estimate. For the exponent $\eta$, we quote here the most recent results: $\eta=-0.395(17),-0.37(5),{ }^{30} \eta=-0.40(4),{ }^{29}$ and $\eta=-0.349(18) .{ }^{28}$ They are all in substantial agreement with our result, which is however significantly more precise. We also mention the estimate $\beta=0.52(9)$, obtained in Ref. 26 by an out-of-equilibrium simulation.

The estimates (93) slightly differ from, and have larger errors than, those obtained in Ref. 32: $\nu=2.53(8)$ and $\eta=-0.384(9)$. There are two reasons for that. First, we have significantly extended the runs for $L=20,24$ for the $\pm J$ model at $p=0.5$. The new results have slightly shifted the estimates of $\beta_{c}, R_{\xi}^{*}$, and of the critical exponents. Second, we have been more conservative. With our present error bars, the estimates are fully consistent with the results of all analyses for the three models we considered.

We also analyzed our data by using the extended-scaling scheme proposed in Ref. 29. This approach might partly take into account the scaling corrections arising from the analytic dependence of the scaling fields on the reduced temperature but it neglects the nonanalytic corrections arising from the irrelevant operators. In some cases, for instance for the overlap susceptibility, this scheme shows an apparent improvement of the scaling behavior with respect to the naive approach in which the analytic corrections are simply neglected. However, the approximate expressions which follow from the extended-scaling scheme are not 
sufficiently precise for a high-precision study of the critical behavior: if one aims at accurate estimates, it is necessary to determine the corrections directly from the data.

\section{APPENDIX A: FINITE-SIZE BEHAVIOR OF THE PHENOMENOLOGICAL COUPLINGS}

We now provide a detailed proof of Eq. (30) for the phenomenological couplings $U_{4}, U_{22}$, and $R_{\xi} \equiv \xi / L$. First, we present a simple physical argument that clarifies the origin of the function $\bar{u}_{h}(t)$ introduced in Eq. (27), and then, a more formal argument based on the Wegner expansion. ${ }^{36}$

A short and physical proof goes as follows. First, note that $\chi$ is not RG invariant and, in particular, it is not invariant under field redefinitions. Given a theory with fields $\phi$, define

$$
\chi_{\phi}=\int d^{3} x[\langle\phi(x) \phi(0)\rangle] .
$$

If we change variables $\phi=Z \psi$, we obtain

$$
\chi_{\phi}=Z^{2} \int d^{3} x[\langle\psi(x) \psi(0)\rangle]=Z^{2} \chi_{\psi} .
$$

Thus, under a field redefinition $\chi \rightarrow Z^{2} \chi$. The function $\bar{u}_{h}(t)$ is exactly the field redefinition that relates the bare lattice fields to the renormalized fields. Hence, we expect at criticality

$$
\chi=\bar{u}_{h}^{2} L^{2-\eta} .
$$

A $t$ dependent prefactor is absent in the phenomenological couplings, because these quantities are RG invariant and in particular, they do not depend on the normalization of the fields. This is quite obvious from their definitions. In the continuum limit they can be written as

$$
\begin{aligned}
U_{4} & \equiv \frac{\int d x_{1} d x_{2} d x_{3} d x_{4}\left[\left\langle\phi\left(x_{1}\right) \phi\left(x_{2}\right) \phi\left(x_{3}\right) \phi\left(x_{4}\right)\right\rangle\right]}{\left[\int d x_{1} d x_{2}\left\langle\phi\left(x_{1}\right) \phi\left(x_{2}\right)\right\rangle\right]^{2}} \\
U_{22} & \equiv \frac{\int d x_{1} d x_{2} d x_{3} d x_{4}\left[\left\langle\phi\left(x_{1}\right) \phi\left(x_{2}\right)\right\rangle\left\langle\phi\left(x_{3}\right) \phi\left(x_{4}\right)\right\rangle\right]}{\left[\int d x_{1} d x_{2}\left\langle\phi\left(x_{1}\right) \phi\left(x_{2}\right)\right\rangle\right]^{2}}-1 \\
\xi^{2} & \equiv \frac{1}{6} \frac{\int d x_{1} d x_{2}\left(x_{1}-x_{2}\right)^{2}\left[\left\langle\phi\left(x_{1}\right) \phi\left(x_{2}\right)\right\rangle\right]}{\int d x_{1} d x_{2}\left\langle\phi\left(x_{1}\right) \phi\left(x_{2}\right)\right\rangle}
\end{aligned}
$$

The formula we report for $\xi$ correspond to Eq. (14) for $L \rightarrow \infty$, disregarding corrections of order $L^{-2}$. Since the number of fields in the denominator is equal to the number of fields in the numerator in all expressions, each quantity is invariant under field redefinitions.

This discussion clarifies the origin of the $t$-dependent prefactor in Eq. (A3) and is at the basis of the statement, which is well accepted in the literature, that $U_{4}\left(T_{c}\right), U_{22}\left(T_{c}\right)$, and $\xi\left(T_{c}\right) / L$ approach universal constants as $L \rightarrow \infty$. The presence of an analytic prefactor would violate universality. Indeed, imagine that a phenomenological coupling $R$ behaves as

$$
R(\beta, L)=\bar{u}_{h}(t)^{\text {some power }} g\left(u_{t} L^{1 / \nu}\right)+\text { scaling corrections }
$$


close to the critical point. Since $\bar{u}_{h}(t)$ is model dependent, at the critical point $R\left(\beta_{c}, L\right)$ would not be universal for $L \rightarrow \infty$. Hence, on purely physical grounds, there must be no $t$-dependent prefactor in any phenomenological coupling, hence no analytic corrections.

This short discussion shows that $R_{\xi}$ and $\chi / L^{2-\eta}$ are conceptually two very different objects. The first quantity is RG invariant and shows a universal FSS behavior. The second quantity is not $\mathrm{RG}$ invariant and, for instance, $\chi\left(T_{c}\right) / L^{2-\eta}$ converges to a model-dependent constant as $L \rightarrow \infty$.

Eq. (30) can also be derived from the usual Wegner's scaling expression for the free energy. Let us first consider $U_{4}$. Using Eq. (26), we find

$$
\begin{aligned}
& {\left[\mu_{4}\right]=\left.L^{d} \frac{\partial^{4} \mathcal{F}}{\partial h^{4}}\right|_{h=0}=L^{4 y_{h}} \bar{u}_{h}^{4} f^{(4)}\left(0, u_{t} L^{y_{t}}\right)+\cdots} \\
& {\left[\mu_{2}\right]=\left.L^{d} \frac{\partial^{2} \mathcal{F}}{\partial h^{2}}\right|_{h=0}=L^{2 y_{h}} \bar{u}_{h}^{2} f^{(2)}\left(0, u_{t} L^{y_{t}}\right)+\cdots}
\end{aligned}
$$

[the dots correspond to nonanalytic scaling corrections and bulk contributions, and the derivatives refer to the first variable appearing in the scaling function $f(x, y)$ ] so that

$$
U_{4}=\frac{f^{(4)}\left(0, u_{t} L^{y_{t}}\right)}{f^{(2)}\left(0, u_{t} L^{y_{t}}\right)^{2}}+\cdots,
$$

which proves Eq. (30).

To discuss $U_{22}$ one should generalize Wegner's scaling expression (see Sec. 3.1 of Ref. 46 for a detailed discussion). Define $Z(\beta, h, L)$ as the partition function of two systems at inverse temperature $\beta$ defined on a lattice of size $L^{3}$ coupled by an interaction

$$
h \sum_{x} \sigma_{1 x} \sigma_{2 x}
$$

Then, consider

$$
\mathcal{F}\left(\beta, h_{1}, h_{2}, L\right)=L^{-d}\left[\ln Z\left(\beta, h_{1}, L\right) \ln Z\left(\beta, h_{2}, L\right)\right] .
$$

A scaling Ansatz like Eq. (25) allows one to obtain an expression analogous to that obtained for $U_{4}$ and to prove Eq. (30) for $U_{22}$.

In order to determine the scaling behavior of $R_{\xi}$ we consider a momentum-dependent magnetic field. The argument goes as follows: Define $Z(\beta, h, L, p)$ as the partition function of two systems at inverse temperature $\beta$ defined on a lattice of size $L^{3}$ coupled by an interaction $h \sum_{x} \sigma_{1 x} \sigma_{2 x} \cos (p \cdot x)$. Then, consider the corresponding disorder-averaged free-energy density

$$
\mathcal{F}(\beta, h, L, p)=L^{-d}[\ln Z(\beta, h, L, p)] .
$$

Under RG transformations $L \rightarrow \lambda L$, momenta scale as $p \rightarrow p / \lambda$, so that the singular part of the free-energy density scales as

$$
\mathcal{F}_{\text {sing }}(\beta, h, L, p)=L^{-d} f\left(p L, u_{h}(h, t, p) L^{y_{h}}, u_{t}(h, t, p) L^{y_{t}}\right),
$$

where we have neglected the nonanalytic scaling corrections and now the scaling fields depend also on $p$. Taking derivatives with respect to $h$ and then setting $h=0$, we obtain for the two-point function [of course $u_{h}(h, t,-p)=u_{h}(h, t, p)$ ]

$$
\widetilde{G}(p)=\bar{u}_{h}(t, p)^{2} L^{2-\eta} f^{(2)}\left(p L, 0, u_{t}(0, t, p) L^{y_{t}}\right),
$$


where we write as before

$$
u_{h}(h, t, p)=h \bar{u}_{h}(t, p)+O\left(h^{3}\right),
$$

and we have neglected subleading terms. For $p \rightarrow 0$, because of the cubic symmetry of the lattice, we have

$$
\begin{aligned}
& \bar{u}_{h}(t, p)=\bar{u}_{h}(t)+O\left(p^{2}\right) \\
& u_{t}(0, t, p)=u_{t}(0, t)+O\left(p^{2}\right)
\end{aligned}
$$

where $\bar{u}_{h}(t)$ and $u_{t}(0, t)$ are the usual (zero-momentum) scaling fields. Hence, for $p \rightarrow 0$, disregarding corrections of order $p^{2}$, we can express $\widetilde{G}(p)$ in terms of the scaling fields that appear for $p=0$ :

$$
\widetilde{G}(p)=\bar{u}_{h}(t)^{2} L^{2-\eta} f^{(2)}\left(p L, 0, u_{t}(0, t) L^{y_{t}}\right)+O\left(p^{2}\right)+\cdots
$$

In the definition (14) of the correlation length $\xi$ we should consider $p=q \sim 1 / L$. Thus, disregarding terms of order $L^{-2}$ we have

$$
\frac{\widetilde{G}(0)-\widetilde{G}(p)}{\widetilde{G}(p)}=\frac{f^{(2)}\left(0,0, u_{t}(0, t) L^{y_{t}}\right)}{f^{(2)}\left((2 \pi, 0,0), 0, u_{t}(0, t) L^{y_{t}}\right)}-1=\Phi\left(u_{t}(0, t) L^{y_{t}}\right)
$$

Neglecting again corrections of order $1 / L^{2}$, we have

$$
\frac{\xi^{2}}{L^{2}}=\frac{1}{4 \pi^{2}} \Phi\left(u_{t}(0, t) L^{y t}\right)
$$

which proves Eq. (30). If we consider the corrections to scaling, this derivation shows that $R_{\xi}$ behaves essentially as $U_{22}$ and $U_{4}$. The only difference is the presence of corrections due to the momentum-dependence of the scaling fields and to the specific definition of the correlation length: they scale as $L^{-2}, L^{-4}, \ldots, L^{-\omega-2}, \ldots$ Since $\omega \approx 1$, in Eq. (30) they represent additional subleading corrections and can thus be neglected. This allows us to consider $R_{\xi}, U_{22}$, and $U_{4}$ on the same footing.

\section{APPENDIX B: SOME TECHNICAL DETAILS ON THE MC SIMULATIONS}

In our MC simulations we implement the standard Metropolis algorithm with a sequential update of the spins. We use a multispin ${ }^{76}$ implementation, in which $n_{\text {bit }}=64$ systems are simulated in parallel. For each of them we use a different set of bonds $\left\{J_{x y}\right\}$.

For the random numbers we use the SIMD-oriented Fast Mersenne Twister (SFMT) ${ }^{77}$ generator. In particular, we use the genrand_res53() function that produces double-precision output. Independent random numbers are employed to generate the starting configurations for each disorder realization and in the parallel-tempering updates. In the latter case very few random numbers are used and thus, it takes virtually no extra time to use individual random numbers for each of the $n_{\text {bit }}$ systems which are simulated in parallel. On the other hand, in order to save CPU time, we use the same sequence of random numbers for the local Metropolis update of any of the $n_{\text {bit }}$ systems. Though this choice does not lead to wrong estimates of the expectation values, it might create a statistical correlation among the $n_{\text {bit }}$ systems. However, since each of the $n_{\text {bit }}$ systems correspond to a different set of 
bond couplings $J_{x y}$ we expect this effect to be negligible. Nevertheless, in order to ensure a correct estimate of the statistical error, in our jackknife analysis we put all $n_{\text {bit }}$ systems that use the same sequence of random numbers in the same bin. In order to compute overlap observables, we performed runs for two systems with the same set $\left\{J_{x y}\right\}$ in parallel. In our MC simulations a single Metropolis update of a single spin takes about $1.2 \times 10^{-9}$ seconds on an Opteron CPU running at $2 \mathrm{GHz}$ (this should be compared with the speed of the dedicated computer Janus, ${ }^{33}$ the fastest computer simulating discrete spin models, which takes $2 \times 10^{-11}$ seconds to update an Ising spin).

To reduce autocorrelations we used the random-exchange or parallel-tempering method. ${ }^{68}$ To this end, we divided the interval $\left[\beta_{\min }, \beta_{\max }\right]$ into $N_{\beta}-1$ equal intervals $\Delta \beta$. The parameter $\beta_{\max }$ was chosen such that $\xi\left(\beta_{\max }\right) / L \approx 0.63$ in most cases; in the latest runs we considered larger values, such that $\xi\left(\beta_{\max }\right) / L \approx 0.66$. The parameter $\beta_{\min }$ was chosen such that $\xi\left(\beta_{\text {min }}\right) \ll L$. We computed the observables in the neighborhood of $\beta_{\text {max }}$ by using their second-order Taylor expansion around $\beta_{\max }$. The coefficients of the expansion were estimated by measuring appropriate correlators with the energy.

An elementary update unit consists in $n_{\text {met }}$ Metropolis sweeps followed by a replica exchange of all pairs of systems at nearby temperatures. The different systems were sequentially visited, starting from those at $\beta_{\min }$ and $\beta_{\min }+\Delta \beta$. As a candidate for the exchange, we considered one of two replicas with equal probability. The acceptance probability for the exchange is $\min [1, \exp (-\Delta \beta \Delta H)]$. Since the measurement of the energy in our implementation costs more CPU time than a Metropolis sweep, we chose $n_{\text {met }} \gg 1$ independent of $\beta$.

The computation of disorder averages of products of thermal expectations requires particular care. Indeed, naive estimators show a bias which may become larger than statistical errors. ${ }^{69}$ To avoid the problem we consider essentially bias-free estimators, defined following Ref. 46. For this purpose we divide the measurement phase of the run into 12 intervals. Between each pair of subsequent interval there is a decorrelation phase. In total, the run consists of the following phases: $E q, D_{1} M_{1}, D_{2} M_{2}, \ldots, D_{12} M_{12}$. After some tests, we fixed the number of update steps for each of them. The equilibration phase $E q$ corresponds to $20 n_{\text {temp }}$ elementary update units; the measurement phases $M_{i}$ correspond to $n_{\text {temp }}$ update units, while the length of $D_{i}$ is $n_{\text {temp }}$ for $i \neq 7$ and $5 n_{\text {temp }}$ for $i=7$. Recall that each elementary update unit corresponds to $n_{\text {met }}$ Metropolis sweeps of all systems and to one full tempering sweep.

The presence of different measurement phases allows us to define bias-free quantities. To define $[\langle A\rangle\langle B\rangle]$ (for instance, this is relevant for the computation of $U_{22}$ ) we average over the samples the quantity

$$
\frac{1}{2 \times 6 \times 6} \sum_{i=1}^{6} \sum_{j=7}^{12}\left[\mu(A)_{i} \mu(B)_{j}+\mu(A)_{j} \mu(B)_{i}\right],
$$

where $\mu(A)_{i}$ is the average of estimates of $A$ obtained in the measurement phase $M_{i}$. Analogously, to compute $[\langle A\rangle\langle B\rangle\langle C\rangle]$ (these correlators are necessary to compute the coefficients of the Taylor expansions around a given value of $\beta$ ), we average over the samples the quantity

$$
\frac{1}{3 ! \times 4^{3}} \sum_{i=1}^{4} \sum_{j=5}^{8} \sum_{k=9}^{12}\left[\mu(A)_{i} \mu(B)_{j} \mu(C)_{k}+5 \text { permutations }\right] \text {. }
$$


TABLE IV: Summary of the parameters for the runs at $p=0.5 . N_{\beta}$ is the number of $\beta$ values used in the parallel-tempering simulation. The rest of the notation is explained in the text. The CPU time refers to a single core of a dual core Opteron CPU running at $2.4 \mathrm{GHz}$.

\begin{tabular}{rrrrrllr}
\hline \hline$L$ & samples $/ 64$ & $n_{\text {met }}$ & $n_{\text {temp }}$ & $N_{\beta}$ & $\beta_{\min }$ & $\beta_{\max }$ & CPU time(days) \\
\hline 4 & 100000 & 5 & 40 & 5 & 0.58 & 0.92 & \\
5 & 100000 & 5 & 50 & 5 & 0.58 & 0.908 & 1 \\
6 & 100000 & 5 & 50 & 5 & 0.58 & 0.9018 & 3 \\
7 & 119103 & 5 & 80 & 5 & 0.58 & 0.899 & 4 \\
8 & 100000 & 5 & 80 & 5 & 0.58 & 0.8975 & 27 \\
9 & 110850 & 10 & 100 & 8 & 0.55 & 0.8962 & 50 \\
10 & 100681 & 10 & 150 & 8 & 0.55 & 0.896 & 183 \\
11 & 109779 & 10 & 300 & 10 & 0.54 & 0.8955 & 308 \\
12 & 106812 & 10 & 400 & 10 & 0.54 & 0.8955 & 210 \\
13 & 38282 & 10 & 600 & 10 & 0.54 & 0.8955 & 361 \\
14 & 31600 & 50 & 200 & 10 & 0.62 & 0.8955 & 830 \\
16 & 24331 & 10 & 1000 & 20 & 0.52 & 0.895 & 658 \\
20 & 1542 & 20 & 2000 & 32 & 0.5125 & 0.895 & 1146 \\
20 & 2291 & 50 & 1500 & 20 & 0.625 & 0.91 & 826 \\
24 & 717 & 25 & 2500 & 32 & 0.5125 & 0.895 & 7874 \\
24 & 1627 & 50 & 2000 & 20 & 0.625 & 0.91 & 782 \\
28 & 285 & 60 & 2500 & 20 & 0.6575 & 0.895 & \\
\hline \hline
\end{tabular}

In order to check equilibration, and decorrelation for the bias correction, we followed the suggestion of Ref. 30. We doubled the length of the run until the estimates of all observables were consistent within error bars. We performed this check only for the observables at $\beta_{\max }$, because these are expected to be the most difficult ones for equilibration and decorrelation. Starting from disordered configurations, we determined the number of update steps $n_{\text {half }}$ that are needed to reach (averaged over samples) half of the equilibrium value of the overlap susceptibility. In total, the equilibration consisted of at least $100 n_{\text {half }}$ update steps. Using these methods to check equilibration, we came up with the choices summarized in Table IV. The parameters are not highly tuned, since we had the CPU time available on short notice. The runs that were done later have typically a larger $\beta_{\min }$ than those done earlier. The run for $L=28$ is a bit at the edge of the criterion given above for equilibration. However, given the rather small number of samples $\left(N_{s}=18240\right)$, we are quite confident that the estimates are correct within the quoted error bars.

1 S. F. Edwards and P. W. Anderson, J. Phys. F 5, 965 (1975).

2 A. Ito, H. Aruga, E. Torikai, M. Kikuchi, Y. Syono, H. Takei, Phys. Rev. Lett. 57, 483 (1986).

3 K. Gunnarsson, P. Svedlindh, P. Nordblad, L. Lundgren, H. Aruga, and A. Ito, Phys. Rev. B 43, 8199 (1991).

4 S. Nair and A. K. Nigam, Phys. Rev. B 75, 214415 (2007). 
5 A. T. Ogielski and I. Morgenstern, Phys. Rev. Lett. 54, 928 (1985).

6 A. T. Ogielski, Phys. Rev. B 32, 7384 (1985).

7 W. L. McMillan, Phys. Rev. B 31, 340 (1985).

8 A. J. Bray and M. A. Moore, Phys. Rev. B 31, 631 (1985).

9 R. N. Bhatt and A. P. Young, Phys. Rev. Lett. 54, 924 (1985).

10 R. R. P. Singh and S. Chakravarty, Phys. Rev. Lett. 57, 245 (1986).

11 J. D. Reger and A. Zippelius, Phys. Rev. Lett. 57, 3225 (1986).

12 R. N. Bhatt and A. P. Young, Phys. Rev. B 37, 5606 (1988).

13 E. Marinari, G. Parisi, and F. Ritort, J. Phys. A 27, 2687 (1994).

14 N. Kawashima and A. P. Young, Phys. Rev. B 53, R484 (1996).

15 L. W. Bernardi, S. Prakash, and I. A. Campbell, Phys. Rev. Lett. 77, 2798 (1996).

16 D. Iñiguez, G. Parisi, and J. J. Ruiz-Lorenzo, J. Phys. A 29, 4337 (1996).

17 B. A. Berg and W. Janke, Phys. Rev. Lett. 80, 4771 (1998).

18 E. Marinari, G. Parisi, and J. J. Ruiz-Lorenzo, Phys. Rev. B 58, 14852 (1998).

19 P. O. Mari and I. A. Campbell, Phys. Rev. E 59, 2653 (1999).

20 M. Palassini and S. Caracciolo, Phys. Rev. Lett. 82, 5128 (1999).

21 H. G. Ballesteros, A. Cruz, L. A. Fernández, V. Martín-Mayor, J. Pech, J. J. Ruiz-Lorenzo, A. Tarancón, P. Téllez, C. L. Ullod, and C. Ungil, Phys. Rev. B 62, 14237 (2000).

22 P. O. Mari and I. A. Campbell, Phys. Rev. B 65, 184409 (2002).

23 T. Nakamura, S.-i. Endoh, and T. Yamamoto, J. Phys. A 36, 10895 (2003).

24 D. Daboul, I. Chang, and A. Aharony, Eur. Phys. J. B 41, 231 (2004).

25 M. Pleimling and I. A. Campbell, Phys. Rev. B 72, 184429 (2005).

26 S. Perez Gaviro, J. J. Ruiz-Lorenzo, and A. Tarancón, J. Phys. A 39, 8567 (2006).

27 F. Parisen Toldin, A. Pelissetto, and E. Vicari, J. Stat. Mech.: Theory Exp. P06002 (2006).

28 T. Jörg, Phys. Rev. B 73, 224431 (2006).

29 I. A. Campbell, K. Hukushima, and H. Takayama, Phys. Rev. Lett. 97, 117202 (2006).

30 H. G. Katzgraber, M. Körner, and A. P. Young, Phys. Rev. B 73, 224432 (2006).

31 J. Machta, C. M. Newman, and D. L. Stein, J. Stat. Phys. 130, 113 (2008); arXiv:0805.0794.

32 M. Hasenbusch, A. Pelissetto, and E. Vicari, J. Stat. Mech.: Theory Exp. L02001 (2008).

33 F. Belletti, M. Cotallo, A. Cruz, L. A. Fernández, A. Gordillo-Guerrero, M. Guidetti, A. Maiorano, F. Mantovani, E. Marinari, V. Martín-Mayor, A. Muñoz Sudupe, D. Navarro, G. Parisi, S. Perez-Gaviro, J. J. Ruiz-Lorenzo, S. F. Schifano, D. Sciretti, A. Tarancón, R. Tripiccione, J. L. Velasco, D. Yllanes (the Janus Collaboration), Comp. Phys. Comm. 178, 208 (2008).

34 K. H. Chen and T. C. Lubensky, Phys. Rev. B 16, 2106 (1977).

35 F. Liers, J. Lukic, E. Marinari, A. Pelissetto, and E. Vicari, Phys. Rev. B 76, 174423 (2007).

36 F. J. Wegner, in Phase Transitions and Critical Phenomena, edited by C. Domb and M. S. Green (Academic Press, New York, 1976), Vol. 6.

37 I. A. Campbell, K. Hukushima, and H. Takayama, Phys. Rev. B 76, 134421 (2007).

38 H. Nishimori, Prog. Theor. Phys. 66, 1169 (1981).

39 A. Georges, D. Hansel, P. Le Doussal, and J. Bouchaud, J. Phys. (Paris) 46, 1827 (1985).

40 P. Le Doussal and A. B. Harris, Phys. Rev. Lett. 61, 625 (1988); Phys. Rev. B 40, 9249 (1989).

41 H. Nishimori, Statistical Physics of Spin Glasses and Information Processing: An Introduction (Oxford University Press, Oxford, 2001).

42 M. Hasenbusch, F. Parisen Toldin, A. Pelissetto, and E. Vicari, Phys. Rev. B 76, 184202 (2007).

43 Y. Deng and H. W. J. Blöte, Phys. Rev. E 68, 036125 (2003). 
44 M. Hasenbusch, F. Parisen Toldin, A. Pelissetto, and E. Vicari, Phys. Rev. B 76, 094402 (2007).

45 M. Campostrini, A. Pelissetto, P. Rossi, and E. Vicari, Phys. Rev. E 65, 066127 (2002).

46 M. Hasenbusch, F. Parisen Toldin, A. Pelissetto, and E. Vicari, J. Stat. Mech.: Theory Exp. P02016 (2007).

47 A. Pelissetto and E. Vicari, Phys. Rep. 368, 549 (2002).

48 G. Toulouse, J. Physique Lettres 41, 447 (1980).

49 N. Kawashima and H. Rieger, in Frustrated Spin Systems, edited by H. T. Diep (World Scientific, Singapore, 2004); cond-mat/0312432.

50 H. Nishimori, J. Phys. Soc. Japan 55, 3305 (1986).

51 H. Kitatani, J. Phys. Soc. Japan 61, 4049 (1992).

52 C. Wang, J. Harrington, and J. Preskill, Ann. Phys. 303, 31 (2003).

53 C. Amoruso and A. K. Hartmann, Phys. Rev. B 70, 134425 (2004).

54 M. Picco, A. Honecker, and P. Pujol, J. Stat. Mech.: Theory Expt. P09006 (2006).

55 A. K. Hartmann, Phys. Rev. B 59, 3617 (1999).

56 It can be shown rigorously that the Nishimori line never intersects the spin-glass phase, $\mathrm{H}$. Kitatani, J. Phys. Soc. Japan 63, 2070 (1994). Since we must also have $p_{F G} \leq p^{*}$, the mixed phase, if it exists, should be confined to the region below the Nishimori line and on the left of the line $p=p^{*}$ (see Fig. 1).

57 D. Sherrington and S. Kirkpatrick, Phys. Rev. Lett. 35, 1792 (1975).

58 T. Castellani, F. Krzakala, and F. Ricci Tersenghi, Eur. Phys. J. B 47, 99 (2005).

59 F. Krzakala and O. C. Martin, Phys. Rev. Lett. 89, 267202 (2002).

60 S. Boettcher and E. Marchetti, Phys. Rev. B 77, 100405(R) (2008).

61 S. Boettcher and J. Davidheiser, Phys. Rev. B 77, 214432 (2008).

62 C. D. Lorenz and R. M. Ziff, Phys. Rev. E 57, 230 (1998).

63 T. Jörg and F. Ricci-Tersenghi, Phys. Rev. Lett. 100, 177203 (2008).

64 V. Privman, in Finite Scaling and Numerical Simulations of Statistical Systems, edited by V. Privman (World Scientific, Singapore, 1990).

65 J. Salas and A. D. Sokal, J. Stat. Phys. 98, 551 (2000).

66 M. Campostrini, M. Hasenbusch, A. Pelissetto, and E. Vicari, Phys. Rev. B 74, 144506 (2006).

67 M. Hasenbusch, J. Phys. A 32, 4851 (1999).

68 C. J. Geyer in Computer Science and Statistics: Proc. of the 23rd Symposium on the Interface, edited by E. M. Keramidas (Interface Foundation, Fairfax Station, 1991), p. 156; K. Hukushima and K. Nemoto, J. Phys. Soc. Jpn. 65, 1604 (1996); for a review, see D. J. Earl and M. W. Deem, Phys. Chem. Chem. Phys. 7, 3910 (2005).

69 H.G. Ballesteros, L. A. Fernández, V. Martín-Mayor, A. Muñoz Sudupe, G. Parisi, and J. J. Ruiz-Lorenzo, Nucl. Phys. B 512, 681 (1998).

70 In practice, we write $R(L, \beta)=R\left(L, \beta_{\text {run }}\right)+a_{R}(L)\left(\beta-\beta_{\text {run }}\right)+b_{R}(L)\left(\beta-\beta_{\text {run }}\right)^{2}$, where $\beta_{\text {run }}$ is the value of $\beta$ closest to $\beta_{c}$ we have considered in the simulation (typically $\beta_{\text {run }}=\beta_{\max }$, see Sec. IV) and $R\left(L, \beta_{\text {run }}\right), a_{R}(L)$, and $b_{R}(L)$ are determined in the simulation. Then, $\beta_{c}, b$, and $R^{*}$ are determined by minimizing the usual $\chi^{2}$ variable.

71 P. Calabrese, V. Martín-Mayor, A. Pelissetto, and E. Vicari, Phys. Rev. E 68, 036136 (2003).

72 In the fit we minimize $\sum_{i}\left[\Xi_{i}-P_{n}\left(x_{i}\right)\right]^{2} / \sigma_{i}^{2}$, where $\Xi=(\xi / L)^{-n / \nu}$, the sum is over all data points, $\sigma_{i}=(n / \nu) \Xi_{i} \Delta \xi_{i} / \xi_{i}$, and $\Delta \xi_{i}$ is the error on $\xi_{i}$. Errors are obtained by using a jackknife procedure and take into account the statistical correlations among the data. At fixed $\nu$ and $\beta_{c}$ the fit is linear and the result is obtained by inverting the least-square matrix. This matrix is 
ill-conditioned (the condition number is of order $10^{24}-10^{32}$, depending on $n$ ). This required the use of 128-digit arithmetics in the calculations.

73 S. Caracciolo, R. Edwards, S. J. Ferreira, A. Pelissetto, and A. D. Sokal, Phys. Rev. Lett. 74, 2969 (1995).

74 The finite thermodynamic limit of $G_{22}$ indicates that self-averaging holds in the hightemperature phase. Therefore, as the lattices become larger at a given temperature, it becomes convenient to improve the statistical accuracy of the expectation values for a given sample. To this end we simulate $n>2$ copies of the system with the same couplings $J_{x y}$. We compute the overlap from all $n(n-1) / 2$ pairs.

75 M. Hasenbusch, A. Pelissetto, and E. Vicari, J. Stat. Mech.: Theory Exp. P11009 (2007).

76 See, e.g., S. Wansleben, J.B. Zabolitzky, and C. Kalle, J. Stat. Phys. 37, 271 (1984); G. Bhanot, D. Duke, and R. Salvador, Phys. Rev. B 337841 (1986).

77 The numerical program and a detailed description can be found at "http://www.math.sci.hiroshima-u.ac.jp/ m-mat/MT/SFMT/index.html". 